Computer Physics Communications, Volume 239, June 2019, pp. 283-310

DOI:10.1016/j.cpc.2018.07.026

\title{
A generalised and low-dissipative multi-directional characteristics-based scheme with inclusion of the local Riemann problem investigating incompressible flows without free-surfaces
}

\author{
Tom-Robin Teschner ${ }^{\mathrm{a}}$, László Könözsy ${ }^{\mathrm{a}, *}$, Karl W. Jenkins ${ }^{\mathrm{a}}$ \\ ${ }^{a}$ Cranfield University, College Road, Cranfield, Bedfordshire, MK43 0AL, United \\ Kingdom
}

\begin{abstract}
In the present study, we develop a generalised Godunov-type multi-directional characteristics-based (MCB) scheme which is applicable to any hyperbolic system for modelling incompressible flows. We further extend the MCB scheme to include the solution of the local Riemann problem which leads to a hybrid mathematical treatment of the system of equations. We employ the proposed scheme to hyperbolic-type incompressible flow solvers and apply it to the Artificial Compressibility (AC) and Fractional-Step, Artificial Compressibility with Pressure Projection (FSAC-PP) method. In this work, we show that the MCB scheme may improve the accuracy and convergence properties over the classical single-directional characteristics-based (SCB) and non-characteristic treatments. The inclusion of a Riemann solver in conjunction with the MCB scheme is capable of reducing the number of iterations up to a factor of 4.7 times compared to a solution when a Riemann solver is not included. Furthermore, we found that both the AC and FSACPP method showed similar levels of accuracy while the FSAC-PP method converges up to 5.8 times faster than the AC method for steady state flows. Independent of the characteristics- and Riemann solver-based treatment of all primitive variables, we found that the FSAC-PP method is 7-200 times faster than the AC method per pseudo-time step for unsteady flows. We investigate low- and high-Reynolds number problems for well-established validation
\end{abstract}

\footnotetext{
${ }^{*}$ Corresponding author.

E-mail address: laszlo.konozsy@cranfield.ac.uk
}

June 26, 2018 
benchmark test cases focusing on a flow inside of a lid driven cavity, evolution of the Taylor-Green vortex and forced separated flow over a backward-facing step. In addition to this, comparisons between a central difference scheme with artificial dissipation and a low-dissipative interpolation scheme have been performed. The results show that the latter approach may not provide enough numerical dissipation to develop the flow at high-Reynolds numbers. We found that the inclusion of a Riemann solver is able to overcome this shortcoming. Overall, the proposed generalised Godunov-type MCB scheme provides an accurate numerical treatment with improved convergence properties for hyperbolic-type incompressible flow solvers.

Keywords: multi-directional characteristics-based scheme; Riemann solver; Godunov-type methods; incompressible flows, steady- and unsteady flows;

\section{PROGRAM SUMMARY}

Program Title: unified2D-C

Licensing provisions: $C C B Y N C 3.0$

Programming language: $C++$

Supplementary material:

Journal reference of previous version:

Does the new version supersede the previous version?:

Reasons for the new version:

Summary of revisions:*

Nature of problem(approx. 50-250 words): Incompressible flow solver have generally a non-hyperbolic type and thus method the method of characteristics and Riemann solvers cannot be used without modifications for low speed flows. In the framework of compressible flows, the Riemann problem - and the method of characteristics which is closely related to it - is an essential part of the solution procedure. The Riemann solver is able to preserve the conservativeness and, through the evaluation of the eigenstructure of the system, introduces transportiveness into the spatial reconstruction schemes. The characteristics-based scheme allows to couple the pressure and velocity in a physical manner which, together with the Riemann solver, presents a new multi-directional Godunov framework for incompressible flows.

Solution method(approx. 50-250 words): We show a generalised description of a multi-directional characteristics-based schemes which may be used with any incompressible and hyperbolic system of equations. The Finite Volume approach is used where inter-cell fluxes are reconstructed through a simple but higher-order polyno- 
mial interpolation scheme which only adds numerical dissipation proportional to its Taylor-series truncation error. We use the Rusanov Riemann solver which provides the needed conservativeness and transportiveness. It also adds just enough numerical dissipation for cases where the dissipation of the numerical scheme is not sufficient while retaining a high level of accuracy.

Additional comments including Restrictions and Unusual features (approx. 50250 words):

* Items marked with an asterisk are only required for new versions of programs previously published in the CPC Program Library.

\section{Introduction}

The solution for simple partial or ordinary differential equations may be straightforward but an increasing complexity of the equations has resulted in creative solution procedures introduced by mathematicians in the past centuries. The method of characteristics is just one such example which simplifies the underlying equations so that an analytic solution can be obtained. It is often attributed to the French mathematician Joseph-Louis Lagrange, however, some historical evidence suggest that it was Paul Charpit, a less wellknown French mathematician, who introduced this method to the Académie in 1784, nine years before Laplace (who was present during Charpit's presentation) passed the information on to Lagrange [1]. Ever since it has found widespread use and played a dominate role in the development of computational fluid mechanics during the second half of the $20^{\text {th }}$ century. Due to the hyperbolic nature of the Euler equations, the application of the method of characteristics remained exclusive to supersonic flows. Hoffman [2] investigated the thrust misalignment in thrust vector controlled nozzles using a three-dimensional characteristic approach. Later, Marcum and Hoffman [3] investigated a simple flow through a nozzle where the transient behaviour was resolved. Cline and Hoffman [4] included chemical reactions and showed that cross flow behaviour was better resolved with their three-dimensional characteristic approach compared to other quasi three-dimensional methods. Ferrari [5] investigated the interference of a wing-body configuration while Delaney and Kavanagh [6] focused on a turbine cascade. Chushkin and Katskova [7], as well as Rakich and Cleary [8] investigated the flow around generic bodies of revolution with blunt noses at different angles of 
attack. Sauerwein [9] further investigated the three-dimensional method of characteristics in conjunction with magnetic fields, chemical reactions, nonequilibrium and multi-component flows for transient regimes.

Initially the computational resources were scarce and hand calculations the only option to obtain results. The unit process, as it is referred to in the literature [10], describes how characteristics fan out from an initial set of conditions and intersect, at which point the primitive variables are updated using a compatibility equation. With an increase in computational resources, however, the process was adopted for computational purposes and sparked the development of several new ways in which characteristics could intersect which has become known under the framework of characteristic networks. An excellent review of various characteristic network can be found in the work of Zucrow and Hoffman [11] and Delaney [12]. Cline and Hoffman [13] investigated several different networks and highlighted their respective advantages while Ransom et al. [14] investigated a second-order characteristic network for a source- and Prandtl-Meyer expansion flow. A comparison of different characteristics-based (CB) approaches has been presented by Roe [15] for the Euler equations. More information on the method of characteristics for compressible flows can be found in Zucrow and Hoffman [16, 11], Delaney [12], Sauerwein [17] and Rusanov [18].

Rusanov [18] showed a generalised form of the method of characteristics for the Euler equations and showed the need to track several characteristic surfaces simultaneously to overcome rank deficient coefficient matrices of the corresponding compatibility equations. This is due to the absence of an independent equation for the pressure which, in the case of the Euler equations, is obtained through the equation of state. This picture is fundamentally different for hyperbolic, incompressible flows where a pseudo pressure equation is constructed, for which then sufficient information are available across at least one characteristic surface.

With the introduction of the Artificial Compressibility method by Chorin [19], the hyperbolic feature was reintroduced into the incompressible flow regime through a pseudo transient time derivative of the pressure. It allowed the usage of the method of characteristics for low-speed flows and was first carried out by Drikakis et al. [20], based on Eberle's CB scheme [21]. Subsequent revision by Neofytou [22] provided a firm theoretical background while $\mathrm{Su}$ et al. [23] showed that both schemes produce similar numerical results, 
albeit Neofytou's scheme being mathematical more rigorous. Shapiro and Drikakis $[24,25]$ provided a comprehensive overview of three different methods to derive the SCB scheme while further extending it to capture variable density flows.

During the same time, Zienkiewicz and Codina [26] and Zienkiewicz et al. [27] introduced the characteristics-based split (CBS) algorithm in the finite-element framework which can be used for both incompressible and compressible flows by either adopting a pressure- or density-based approach. In a three-step procedure, the velocity is first guessed from which either the pressure or density is recovered. In the final step, the velocity is updated based on the new density or pressure field. It resembles the Fractional-Step (FS) or splitting procedure encountered in incompressible flows from which its name is derived. Nithiarasu [28] used the CBS scheme and applied it to steady and unsteady turbulent flows using different RANS models, showing how the scheme can be extended to capture turbulent flows.

In recent years, Razavi et al. [29] and Zamzamian and Razavi [30] introduced a multi-directional characteristics-based (MCB) scheme for the Artificial Compressibility method. It is multi-directional in the sense that characteristic surfaces are considered and multiplied into the governing equations through the chain rule. This approach is consistent with the CB scheme discussed above for compressible flows. However, its multi-directional nature also helps to distinguish itself from the version of Drikakis which is subsequently referred to as the single-directional characteristics-based (SCB) scheme. The single-directional nature is due to the simplification that only characteristic lines are used in the version of Drikakis et al. which can be introduced through a Taylor series in space and time. To treat two- and three-dimensional flows, the procedure is simply applied in each direction which does not take directional information into account and so resorts to an isotropic treatment. Razavi et al. [29] and Zamzamian and Razavi [30] showed favourable convergence and accuracy properties compared to the single-directional version. This has led to further development of the scheme. Fathollahi and Zamzamian [31] increased the number of discrete wave directions - necessary to discretise the compatibility equations - showing an increase in the convergence rate while the accuracy was not affected. Hashemi and Zamzamian [32] and Zamzamian and Hashemi [33] introduced modified far-field and solid boundary conditions in conjunction with the CB scheme treatment while in a further modification, Hashemi and Zamzamian [34] extended the multi-directional approach to unstructured domains. Further ap- 
plications of the MCB applied to heat-transfer and turbulent flows can be found in Razavi and Adibi [35] and Razavi and Hanifi [36].

In the current form, the multi-directional scheme has been derived solely for the Artificial Compressibility method. However, with the recent introduction of the unified Fractional-Step, Artificial Compressibility and Pressure projection (FSAC-PP) method of Könözsy [37], later introduced in [38], a renewed interest in hyperbolic, incompressible methods has surged. The FSAC-PP method unifies Chorin's [19] Artificial Compressibility (AC) method with Chorin's [39] and Témam's [40] Fractional-Step Pressure Projection (FS-PP) method and has shown advantageous accuracy and convergence properties over its baseline methods $[37,38]$. It was originally introduced using the SCB scheme which makes it a Godunov method. It has been further investigated for variable density flows in a Y-junction channel [41], trapping and positioning of cryogenic liquids through acoustic liquid manipulation [42], the vortex pairing problems with comparison to low-Mach corrected compressible solvers [43], forced separation over a backward facing step geometry [44] and different approximate Riemann solvers [45].

The structure of the current work is the following, in Section 2, we review the governing equations of the $\mathrm{AC}$ and the FSAC-PP method and give details of the discretisation procedure. In Section 3, we develop a generalised method of characteristics, applicable to any hyperbolic system of equation for incompressible flows, which we apply to the AC and FSAC-PP method. In Section 4, we introduce the Riemann problem and an approximate Riemann solver and show the relation between Riemann solvers and the method of characteristics. We further demonstrate how to couple both approaches together and discuss results for the lid driven cavity, Taylor-Green vortex and backward facing step problem in Section 5 for various CB scheme and Riemann solver combinations.

\section{Governing Equations}

In this Section, we review the governing equations of the $\mathrm{AC}$ and FSACPP method and show the necessary steps which are required to discretise the equations. 


\subsection{Artificial Compressibility Method (1967)}

The Artificial Compressibility (AC) method of Chorin [19] was the first to introduce the hyperbolic nature into the incompressible flow framework. We use it as a baseline method against which we compare our results. The governing equations are

$$
\begin{aligned}
\frac{1}{\beta} \frac{\partial p}{\partial \tau}+\nabla \cdot \mathbf{U} & =0 \\
\frac{\partial \mathbf{U}}{\partial \tau}+\frac{\partial \mathbf{U}}{\partial t}+(\mathbf{U} \cdot \nabla) \mathbf{U} & =-\frac{1}{\rho} \nabla p+\nu \nabla^{2} \mathbf{U}
\end{aligned}
$$

where the continuity equation is perturbed by a pseudo time derivative of the pressure. Here, $\beta$ accounts for the relation between the pressure and density. Since we cannot determine this relationship through thermodynamic reasoning, the time derivative is non-physical and thus time marching is done in pseudo time where $\beta$ becomes a convergence parameter. Therefore, the time derivative in the momentum equation is transformed into pseudo time as well. The continuity equation only regains a physical meaning once the pseudo time derivative vanishes. In that case, the steady state solution is obtained. If an unsteady solution is sought, the momentum equation has to be augmented by an additional real-time derivative, where the solution is

obtained through a dual-time stepping procedure [46]. Otherwise this term is set to zero.

\subsection{Fractional-Step, Artificial Compressibility and Pressure Projection Method} (2012)

Könözsy [37] provided a new framework in which Chorin's AC method was unified with Chorin's [39] and Témam's [40] Fractional-Step, Pressure projection (FS-PP) method. It is consequently named the FSAC-PP method which incorporates the hyperbolic and elliptic features within the same method. This allows for CB schemes to be applied in the first hyperbolic Fraction-Step, while the elliptic feature can be regarded as a physical smoothing procedure of the pressure which provides extra stability in the second Fractional-Step. In the first Fractional-Step, the perturbed continuity equation of the $\mathrm{AC}$ method is solved alongside the momentum equations of the FS-PP method, in which the pressure gradient term is dropped, which reads in semi-discretised form 


$$
\begin{aligned}
\frac{1}{\beta} \frac{\partial p}{\partial \tau}+\nabla \cdot \mathbf{U} & =0 \\
\frac{\hat{\mathbf{U}}-\mathbf{U}}{\Delta \tau}+\frac{\partial \mathbf{U}}{\partial t}+(\mathbf{U} \cdot \nabla) \mathbf{U} & =\nu \nabla^{2} \mathbf{U}
\end{aligned}
$$

This provides an intermediate velocity field $\hat{\mathbf{U}}$ which does not satisfy the divergence free constrain. In the second Fractional-Step, the pressure gradient is recovered from

$$
\frac{\mathbf{U}^{n+1}-\hat{\mathbf{U}}}{\Delta \tau}=-\frac{1}{\rho} \nabla p^{n+1} .
$$

Taking the divergence of Eq.(5) and constraining the velocity field at time level $n+1$ to be divergence free, i.e. $\nabla \cdot \mathbf{U}^{n+1}=0$, we obtain the following Poisson equation for the pressure

$$
\nabla^{2} p^{n+1}=\frac{\rho}{\Delta \tau} \nabla \cdot \hat{\mathbf{U}}
$$

Numerically speaking, we will not satisfy $\nabla \cdot \mathbf{U}^{n+1}=0$ in each pseudo time step but as the solution converges, $\nabla \cdot \mathbf{U}^{n+1} \rightarrow 0$. With the pressure field at $n+1$ available, we obtain the final velocity field from Eq.(5) as

$$
\mathbf{U}^{n+1}=\hat{\mathbf{U}}-\frac{\Delta \tau}{\rho} \nabla p^{n+1} .
$$

Eqs.(3)-(4) form the first Fractional-Step which is hyperbolic and so a CB scheme can be applied. The second Fractional-Step, Eqs.(5)-(7), is elliptic, which stabilises the pressure field and through Eq.(7) the velocity as well. It is important to highlight here that since an initial pressure field is provided through Eq.(3), it is not necessary to solve Eq.(6) fully and it has been found that ten iterations in the Poisson solver are usually sufficient to accelerate convergence while stabilising the pressure.

\subsection{Numerical implementation details}

The equations are discretised using the finite volume method on a dual control volume, or cell-vertex scheme, as shown in Figure 1. The primitive variables are stored on the vertices while the finite volumes are constructed around the vertices. This ensures that Dirichlet boundary conditions can be 
imposed directly on the boundaries.

Gradients are approximated using Gauss' Theorem as

$$
\begin{array}{r}
\frac{\partial \phi}{\partial x}=\int_{V} \frac{\partial}{\partial x} \phi \mathrm{d} V=\int_{A} \vec{n} \cdot \phi \mathrm{d} A \approx \sum_{\text {faces }} \vec{n} \cdot \phi A= \\
\left(\phi_{i+1 / 2}-\phi_{i-1 / 2}\right) A \frac{V}{V}=\frac{\phi_{i+1 / 2}-\phi_{i-1 / 2}}{\Delta x} V .
\end{array}
$$

Here we made use of the fact that in two dimensions and on a Cartesian grid, $\vec{n} \cdot A=n_{x} \cdot \mathrm{d} y+n_{y} \cdot \mathrm{d} x=1 \cdot \mathrm{d} y+0 \cdot \mathrm{d} x=\mathrm{d} y$ in the $x$-direction and similarly, $\vec{n} \cdot A=\mathrm{d} x$ in the $y$-direction. The volume per unit length is given by $V=\mathrm{d} x \cdot \mathrm{d} y$.

To obtain the second order gradients, we set $\phi=\partial \phi / \partial x$ and insert it into Eq.(8). We obtain

$$
\begin{array}{r}
\frac{\partial^{2} \phi}{\partial x^{2}}=\frac{1}{\Delta x}\left(\left.\frac{\partial \phi}{\partial x}\right|_{i+1 / 2}-\left.\frac{\partial \phi}{\partial x}\right|_{i-1 / 2}\right) V=\frac{1}{\Delta x}\left(\frac{\phi_{i+1}-\phi_{i}}{\Delta x}-\frac{\phi_{i}-\phi_{i-1}}{\Delta x}\right) V= \\
\frac{\phi_{i+1}-2 \phi_{i}+\phi_{i-1}}{(\Delta x)^{2}} V
\end{array}
$$

which reverts to a finite difference approximation. We make use of a third-order polynomial to approximate the variables at the inter-cell face, which is given as [47]

$$
\begin{aligned}
\phi_{i+1 / 2}^{L} & =\frac{5}{6} \phi_{i}-\frac{1}{6} \phi_{i-1}+\frac{1}{3} \phi_{i+1}, \\
\phi_{i+1 / 2}^{R} & =\frac{5}{6} \phi_{i+1}-\frac{1}{6} \phi_{i+2}+\frac{1}{3} \phi_{i} .
\end{aligned}
$$

Razavi et al. [29] and Zamzamian and Razavi [30] used a central scheme with artificial dissipation [48] in their MCB scheme which we use here as a reference scheme. It is defined as

$$
F_{i+1 / 2}^{I}=F^{I}\left(\phi_{i+1 / 2}\right)-D_{i+1 / 2}
$$




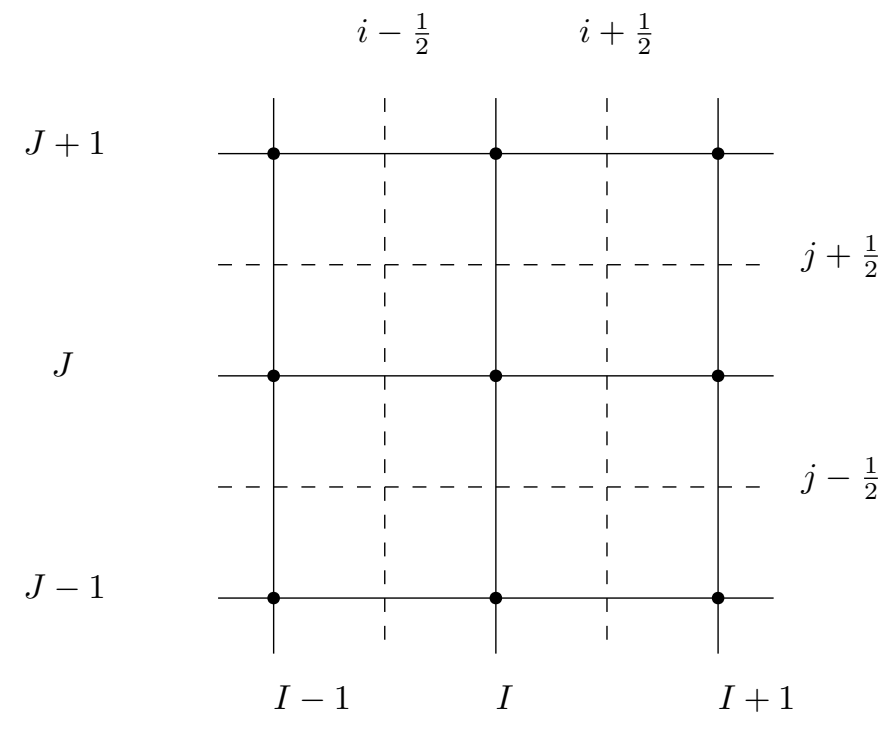

Figure 1: The dual control volume arrangement used in the present work. The solid lines correspond to the mesh while the dashed lines are the constructed finite volumes surrounding each cell vertex.

where $F_{i+1 / 2}^{I}$ denotes the inviscid fluxes, $D_{i+1 / 2}$ is the influence of the artificial dissipation and $\phi_{i+1 / 2}$ is obtained through the central difference (CD) scheme as

$$
\phi_{i+1 / 2}=\frac{1}{2}\left(\phi_{i}+\phi_{i+1}\right) .
$$

The artificial dissipation term itself is obtained as

$$
D_{i+1 / 2}=\frac{1}{2}\left(\Lambda^{L}+\Lambda^{R}\right)\left[k_{2}\left(\phi_{i+1}-\phi_{i}\right)-k_{4}\left(\phi_{i+2}-3 \phi_{i+1}+3 \phi_{i}-\phi_{i-1}\right)\right] .
$$

Here, we set $k_{2}=1 / 2$ and $k_{4}=1 / 128$ and define $\Lambda$ as

$$
\begin{aligned}
\Lambda^{L} & =\left(\left|\mathbf{U}_{i}^{\perp}\right|+\beta\right) A, \\
\Lambda^{R} & =\left(\left|\mathbf{U}_{i+1}^{\perp}\right|+\beta\right) A,
\end{aligned}
$$

where $\mathbf{U}_{i}^{\perp}$ is the velocity component normal to the $i^{\text {th }}$-cell face area. For compressible flows, the constants $k_{2}$ and $k_{4}$ may further be a function of a 
pressure sensor to reduce oscillations near discontinuities [48, 49], however, in the context of incompressible flows it has been found that the pressure sensor term introduces oscillations and thus prohibits the solution to converge fully. For the FSAC-PP method, we follow the approach of Pentaris et al. [50] — who used the FS-PP method in conjunction with the central scheme and artificial dissipation — where we only add artificial dissipation in the first Fractional-Step.

The time stepping in pseudo time is achieved by a third-order Runge-Kutta scheme with TVD properties [51], given by

$$
\begin{aligned}
\mathbf{U}^{*} & =\mathbf{U}^{n}+\Delta \tau \operatorname{RHS}\left(\mathbf{U}^{n}\right), \\
\mathbf{U}^{* *} & =\frac{3}{4} \mathbf{U}^{n}+\frac{1}{4} \mathbf{U}^{*}+\frac{1}{4} \Delta \tau \operatorname{RHS}\left(\mathbf{U}^{*}\right), \\
\mathbf{U}^{n+1} & =\frac{1}{3} \mathbf{U}^{n}+\frac{2}{3} \mathbf{U}^{* *}+\frac{2}{3} \Delta \tau \operatorname{RHS}\left(\mathbf{U}^{* *}\right) .
\end{aligned}
$$

In the dual-time stepping procedure, the real-time derivative is approximated by a second-order backward scheme as

$$
\frac{\partial \mathbf{U}}{\partial t}=\frac{3 \mathbf{U}_{i}^{m+1, n}-4 \mathbf{U}_{i}^{m}+\mathbf{U}_{i}^{m-1}}{2 \Delta t} .
$$

Here, $\mathbf{U}_{i}^{m+1, n}$ is updated in pseudo time along with the pseudo time derivative while $\mathbf{U}_{i}^{m}$ and $\mathbf{U}_{i}^{m-1}$ are updated after each pseudo time step has converged. The time step itself is defined analogous to $[37,38]$ for both pseudo and real-time component

$$
\Delta(\tau, t)=\min \left(\Delta(\tau, t)_{i n v}, \Delta(\tau, t)_{v i s}\right)
$$

where

$$
\begin{aligned}
\Delta(\tau, t)_{i n v} & =\frac{\min (\mathrm{d} x, \mathrm{~d} y) \cdot \mathrm{CFL}_{i n v}}{\sqrt{u^{2}+v^{2}}+\sqrt{u^{2}+v^{2}+\beta}} \\
\Delta(\tau, t)_{v i s} & =\frac{\mathrm{CFL}_{v i s}}{4 \nu \cdot \min \left((\mathrm{d} x)^{2},(\mathrm{~d} y)^{2}\right)}
\end{aligned}
$$

are the time steps due to the inviscid and viscous part of the Navier-Stokes equations, respectively. 


\section{The multi-directional characteristics-based scheme for incom- pressible flows}

In this Section, we generalise the MCB scheme introduced by Razavi et al. [29] for incompressible flows by following the approach of Rusanov [18], who presented a generalised framework for compressible flows. We will first show that there is a fundamental difference when deriving the MCB scheme for incompressible and compressible flows and then apply the method to both the AC and FSAC-PP method.

\subsection{The generalised characteristics-based scheme}

We consider a hyperbolic, incompressible system of equations that is written in the form of

$$
\mathcal{B}_{j} \frac{\partial \mathbf{U}_{j}}{\partial t}+\sum_{i=1}^{n} \frac{\partial \mathbf{F}_{i j}}{\partial \mathbf{x}_{i}}=0 .
$$

Here, $\mathcal{B}_{j}$ is a preconditioning vector, $\mathbf{U}_{j}$ is a vector containing the primitive variables while $\mathbf{F}_{i j}$ contains the inviscid fluxes. The system in Eq.(19) is augmented by a characteristic surface of the shape $f=f\left(t, \mathbf{x}_{i}\right)=0$ which is introduced through the chain rule. It follows that

$$
\mathcal{B}_{j} \frac{\mathrm{d} \mathbf{U}_{j}}{\mathrm{~d} f} \frac{\partial f}{\partial t}+\sum_{i=1}^{n} \frac{\mathrm{d} \mathbf{F}_{i j}}{\mathrm{~d} f} \frac{\partial f}{\partial \mathbf{x}_{i}}=0 .
$$

We can simplify Eq.(20) by multiplying it with $\mathrm{d} f$ and $\mathcal{B}_{j}^{-1}$. Furthermore, we translate the convective flux treatment from a conservative into a nonconservative form $\left(\mathrm{d} \mathbf{F}_{i j}=a_{i j} \mathrm{~d} \mathbf{U}_{j}\right)$ so that we have

$$
\mathrm{d} \mathbf{U}_{j} f_{t}+\sum_{i=1}^{n} \mathcal{B}_{j}^{-1} a_{i j} \mathrm{~d} \mathbf{U}_{j} f_{\mathbf{x}_{i}}=0,
$$

where $\partial f / \partial \tau=f_{\tau}$ and $\partial f / \partial \mathbf{x}_{i}=f_{\mathbf{x}_{i}}$. It is perhaps here that we need to mention a difference in notation to the one used by Rusanov [18]. Zucrow and Hoffman [16] presented four different approaches to describe the method of characteristics, which can be derived from a physical, a purely heuristic, a mathematical and a most rigorous mathematical point of view. While Rusanov's approaches is situated under the most rigorous mathematical approach, using an interior operator in the form of $\mathrm{d}_{\mathbf{U}} f=\mathbf{U} \cdot \nabla f$ of which 
then $m^{1}$ linear combinations are used to derive the compatibility equations, we use elements from both the mathematical and physical approach for our derivation. Returning to Eq.(21), we isolate the primitive variables as

$$
\left(f_{t}+\sum_{i=1}^{n} \mathcal{B}_{j}^{-1} a_{i j} f_{\mathbf{x}_{i}}\right) \mathrm{d} \mathbf{U}_{j}=0
$$

and we define

$$
\mathcal{T}=f_{t}+\sum_{i=1}^{n} \mathcal{B}_{j}^{-1} a_{i j} f_{\mathbf{x}_{i}}
$$

as our coefficient matrix corresponding to our primitive variables in difference form $\mathrm{d} \mathbf{U}_{j}$. Since we have assumed Eq.(19) to be hyperbolic, we ensure that the eigenvalues of $\mathcal{T}$ are all real and distinct for which we can then find real and distinct characteristics. Thus, for a non-trivial solution of Eq.(22), we require that $\operatorname{det}(\mathcal{T}) \neq 0$. This leads to

$$
\operatorname{det}\left(f_{t}+\sum_{i=1}^{n} \mathcal{B}_{j}^{-1} a_{i j} f_{\mathbf{x}_{i}}\right)=\Psi_{k}
$$

where the solution $\Psi_{k}, k=1, \ldots, l$ corresponds to $l$ characteristic surfaces if their corresponding normal vectors satisfy $\mathbf{n} \cdot \mathbf{u}=0$, where $\mathbf{u}$ is the primitive variable vector containing only the velocity components. Thus, Eq.(24) requires the normal vector in space to be perpendicular to the velocity vector which, by definition, lies inside the characteristic surface. If such a condition can be satisfied, then for each characteristic surface $\Psi_{k}$, the rank of the coefficient matrix is computed from

$$
\operatorname{rank}(\mathcal{T})=\operatorname{rank}\left(f_{t}+\sum_{i=1}^{n} \mathcal{B}_{j}^{-1} a_{i j} f_{\mathbf{x}_{i}}\right)=p_{k}\left(\Psi_{k}\right)
$$

where $\mathcal{T}$ in Eq. (25) has to be reformulated in terms of $\Psi_{k}$. It will be shown in Section 3.2 how to accomplish this step for the AC and FSAC-PP method, respectively. At this stage, we do not have any information on the normal vector but can relate it to the surface derivatives $f_{t}$ and $f_{\mathbf{x}_{i}}$. Considering a general surface in space, taking its derivative with respect to time yields

\footnotetext{
${ }^{1} m=$ number of dimensions, including time
} 


$$
\frac{\partial f\left(\mathbf{x}_{i}\right)}{\partial t}=\frac{\partial f}{\partial \mathbf{x}_{i}} \frac{\mathrm{d} \mathbf{x}_{i}}{\mathrm{~d} t}=f_{\mathbf{x}_{i}} \cdot \mathbf{u}_{i}=\nabla f \cdot \mathbf{u}=\mathbf{n} \cdot \mathbf{u}=0 .
$$

Even if the assumed characteristic surface is inhomogeneous, i.e. $f\left(t, \mathbf{x}_{i}\right)=$ $D$, where $D$ is an arbitrary constant, the time derivative of a constant will always be zero and thus we can equate $\nabla f \cdot \mathbf{u}=\mathbf{n} \cdot \mathbf{u}=0$ for which we have shown that $\nabla f=\mathbf{n}$.

For each characteristic surface $k=1, . ., l$, we evaluate

$$
s_{k}=m-p_{k} .
$$

A rank-deficient system results in $s_{k}>0$ while a system of $m$ linear independent equations results in $s_{k}=0$. Rusanov [18] stated that a system of governing equations for compressible flows necessarily results in a rank-deficient system for all characteristic surfaces considered. Hence, a combination of at least two independent characteristic surfaces is required to sufficiently constrain the compatibility equations and Rusanov showed which combinations of primitive variables on different surfaces are valid combinations. For incompressible flows, however, it turns out that at least once $s_{k}=0$ holds so that only one characteristic surface needs to be considered as a consequence. We can summarise this as

$$
s_{k}= \begin{cases}\forall k: s_{k}>0, & \text { compressible } \\ \exists k: s_{k}=0, & \text { incompressible }\end{cases}
$$

This can be understood by the different solution procedure employed by compressible and incompressible flows, respectively. In the case of compressible flows, we have $m+3$ primitive variables but only $m+2$ governing equations. The equation of state is taken to close the system, however, it is not a transport equation and so not considered in Eq.(19). For incompressible flows, however, the equation of state approach does not work and a pseudo pressure equation has to be constructed to close the system. This equation, in turn, is considered in Eq.(19) so that we have the same number of primitive variables and equations to solve for them. Thus, when constructing the compatibility equations, we end up with the constraints given by Eq.(28). This demonstrates the main difference between the compressible and incompressible flow case and shows the simplifications involved when dealing with the method of characteristics for incompressible flows. The final 
compatibility equations are then found from Eq.(22) for each element of the primitive variable vector $\mathrm{U}_{j}$ for the characteristic surface $\Psi_{k}$ for which $s_{k}=0$.

Zucrow and Hoffman [16, 11] showed a basic derivation of the characteristic surfaces. The full derivation, however, is a lengthy process and requires elements of differential geometry. It was omitted in their discussion but a detailed and in-depth derivation was given by Delaney [12]. A similar derivation can be done for the case of incompressible flows, however, Razavi et al. [29] provided a more elegant and shorter derivation which we adopt here. Our starting point is the derivative of the characteristic surface which we can write as, using the chain rule

$$
\frac{\partial f}{\partial t}+\sum_{i=1}^{n} \frac{\mathrm{d} \mathbf{x}_{i}}{\mathrm{~d} t} \frac{\partial f}{\partial \mathbf{x}_{i}}=0 .
$$

We have also found previously that

$$
\mathbf{u} \cdot \mathbf{n}=\Psi_{k} .
$$

Since $\mathrm{d} \mathbf{x}_{i} / \mathrm{d} t=\mathbf{u}$ and $\partial f / \partial \mathbf{x}_{i}=\nabla f=\mathbf{n}$, we can subtract Eq.(29) from Eq.(30) so that

$$
\sum_{i=1}^{n}\left(\mathbf{u}_{i}-\frac{\mathrm{d} \mathbf{x}_{i}}{\mathrm{~d} t}\right) \cdot \mathbf{n}=\Psi_{k},
$$

which is the equation of the characteristic surface $k$. At this point, we split Eq.(31) into each direction so that

$$
\left(\mathbf{u}_{i}-\frac{\mathrm{d} \mathbf{x}_{i}}{\mathrm{~d} t}\right) \cdot \mathbf{n}=\Psi_{k}
$$

corresponds to the component of the characteristic surface in the $\mathbf{x}_{i}$ direction. Although we have given the full derivation of the characteristic surface, it is usually dropped in the literature where only the compatibility equation is considered. In-fact, Rusanov [18] did not give any consideration to the characteristic equation itself and neither did Drikakis et al. [20] for their SCB scheme (although Eq.(32) could be used since it is split into each respective direction). In the literature on the MCB scheme [29, 30, 31, 32, 33, 34, 35, 36], a first- and second-order version has been proposed. In the first-order scheme, the characteristic equation, i.e. Eq.(32) is dropped and values on the pseudo 
Mach cone that are required for the compatibility equations, which can be derived from Eq.(22), are taken from the interpolated values on the cell interfaces. These, in turn, are obtained in the current work through either Eq.(10) or Eq.(11). If the characteristic equation is used, then values need to be interpolated to the intersection of the characteristic surface with the time level $n$ at which either an initial guess or a previous computed solution is available. The accuracy of the interpolation scheme thus determines the order of the overall scheme, although, strictly speaking, this classification only applies to the underlying interpolation scheme and not to the characteristicsbased scheme itself. In the literature on the MCB scheme, a mix of firstand second-order results are presented while we only employ the first-order version in the current work. This has been done to make a fair comparison between the MCB and SCB scheme which, by default, is first-order accurate. It is possible, however, to use a higher-order interpolation scheme to increase the order of the MCB scheme itself, using the definition from the literature.

\subsection{The multi-directional characteristics-based scheme for the $A C$ and FSAC- PP method}

In this Section, we will derive the method of characteristics for the incompressible AC and FSAC-PP method and show how to construct the compatibility equations. It is convenient to do the derivation for the $\mathrm{AC}$ method first. The derivation for the FSAC-PP method is closely related to that of the $\mathrm{AC}$ method for which we will give considerations at the end of this Section.

Corresponding to Eq.(19), our hyperbolic system of equations of the AC method in two space dimensions can be written as

$$
\mathcal{B} \frac{\partial \mathbf{U}}{\partial t}+\frac{\partial \mathbf{F}}{\partial x}+\frac{\partial \mathbf{G}}{\partial y}=0
$$

Here, we have split $\mathbf{F}_{i j}$ into its components in the $x$ - and $y$-direction which are denoted by $\mathbf{F}$ and $\mathbf{G}$, respectively. The components of the vectors are given by

$$
\mathcal{B}=\left[\begin{array}{c}
\frac{1}{\beta} \\
1 \\
1
\end{array}\right], \quad \mathbf{U}=\left[\begin{array}{c}
p \\
u \\
v
\end{array}\right], \quad \mathbf{F}=\left[\begin{array}{c}
u \\
u^{2}+p \\
v u
\end{array}\right], \quad \mathbf{G}=\left[\begin{array}{c}
v \\
u v \\
v^{2}+p
\end{array}\right]
$$


We multiply a surface into Eq.(33) by using the chain rule which becomes our characteristic surface if $\mathbf{n} \cdot \mathbf{u}=0$ is satisfied. This is also shown in Eq.(20) for which our system of equations, now in scalar form, reads

$$
\begin{aligned}
& \frac{1}{\beta} \frac{\mathrm{d} p}{\mathrm{~d} f} \frac{\partial f}{\partial \tau}+\frac{\mathrm{d} u}{\mathrm{~d} f} \frac{\partial f}{\partial x}+\frac{\mathrm{d} v}{\mathrm{~d} f} \frac{\partial f}{\partial y}=0, \\
& \frac{\mathrm{d} u}{\mathrm{~d} f} \frac{\partial f}{\partial \tau}+u \frac{\mathrm{d} u}{\mathrm{~d} f} \frac{\partial f}{\partial x}+v \frac{\mathrm{d} u}{\mathrm{~d} f} \frac{\partial f}{\partial y}+\frac{1}{\rho} \frac{\mathrm{d} p}{\mathrm{~d} f} \frac{\partial f}{\partial x}=0, \\
& \frac{\mathrm{d} v}{\mathrm{~d} f} \frac{\partial f}{\partial \tau}+u \frac{\mathrm{d} v}{\mathrm{~d} f} \frac{\partial f}{\partial x}+v \frac{\mathrm{d} v}{\mathrm{~d} f} \frac{\partial f}{\partial y}+\frac{1}{\rho} \frac{\mathrm{d} p}{\mathrm{~d} f} \frac{\partial f}{\partial y}=0 .
\end{aligned}
$$

We have dropped here the viscous term as there is no generalised theory on how to include the viscous term in the method of characteristic. This is consistent with the literature in which the SCB and MCB has been used and developed. In turn, we can only apply the characteristic variables to the inviscid fluxes. Hoffmann [52] showed how second-order derivatives may be included in the classification of partial differential equations and a similar approach may be taken when deriving the compatibility equations. There are, however, other problems associated to this approach which is outside the scope of the current work.

We multiply Eqs.(35)-(37) by $\mathrm{d} f$, express surface derivatives as $\partial f / \partial \tau=f_{\tau}$, $\partial f / \partial x=f_{x}$ and $\partial f / \partial y=f_{y}$ and rewrite the system of equations in nonconservative form so that we obtain

$$
\begin{array}{r}
\frac{f_{\tau}}{\beta} \mathrm{d} p+f_{x} \mathrm{~d} u+f_{y} \mathrm{~d} v=0, \\
f_{\tau} \mathrm{d} u+u f_{x} \mathrm{~d} u+v f_{y} \mathrm{~d} u+\frac{f_{x}}{\rho} \mathrm{d} p=0, \\
f_{\tau} \mathrm{d} v+u f_{x} \mathrm{~d} v+v f_{y} \mathrm{~d} v+\frac{f_{y}}{\rho} \mathrm{d} p=0 .
\end{array}
$$

We can express the above system in matrix form, corresponding to the matrix $\mathcal{T}$ in Eq.(23) as

$$
\left[\begin{array}{ccc}
f_{\tau} / \beta & f_{x} & f_{y} \\
f_{x} / \rho & \Psi & 0 \\
f_{y} / \rho & 0 & \Psi
\end{array}\right]\left[\begin{array}{l}
\mathrm{d} p \\
\mathrm{~d} u \\
\mathrm{~d} v
\end{array}\right]=\left[\begin{array}{l}
0 \\
0 \\
0
\end{array}\right],
$$


where we have introduced the short-hand notation $\Psi=f_{\tau}+u f_{x}+v f_{y}$. We return to Eq. (24) where we required the determinant of $\mathcal{T}$ to become zero. This can be also understood from a more physical point of view. Characteristic lines are in general defined as those lines along which derivatives may become discontinuous. If we attempt to obtain an expression for the characteristic path $(\mathrm{d} x / \mathrm{d} t$ in time or $\mathrm{d} y / \mathrm{d} x$ in space for steady flows), the solution for the derivatives is most conveniently obtain through Cramer's rule where the coefficient matrix would appear in the denominator of the solution. Thus, for the solution to become discontinuous, the determinant has to become zero. The determinant of $\mathcal{T}$ in Eq.(41) is computed from

$$
\operatorname{det}\left(\left[\begin{array}{ccc}
f_{\tau} / \beta & f_{x} & f_{y} \\
f_{x} / \rho & \Psi & 0 \\
f_{y} / \rho & 0 & \Psi
\end{array}\right]\right)=0,
$$

for which the following solutions are obtained as

$$
\Psi_{1}=0, \quad \Psi_{2}=\frac{\beta}{\rho f_{\tau}}\left(f_{x}^{2}+f_{y}^{2}\right)=\frac{\beta}{\rho f_{\tau}} .
$$

For $\Psi_{2}$ we have used the fact that $\nabla f=\mathbf{n}$, where we have rescaled $\mathbf{n}$ so that $n_{x}^{2}+n_{y}^{2}=1$. If $\Psi_{1}$ and $\Psi_{2}$ do comply with $\mathbf{n} \cdot \mathbf{u}=0$, then we have found two characteristic surfaces and can insert $\Psi_{k}$ into Eq.(41) to obtain the compatibility equations for $\mathrm{d} p, \mathrm{~d} u$ and $\mathrm{d} v$ on the characteristic surface $k$. Unfortunately, we only have $\mathbf{n} \cdot \mathbf{u}=\Psi_{k}$ and $\mathbf{n} \cdot \mathbf{n}=1$ available as two constrains for our three primitive variables $u, v$ and $p$. Thus, the system is under constrained and to prove that $\Psi_{k}$ is indeed a solution to the characteristic surface, we have to consider geometrical constraints as well. We do so by intersecting $\mathbf{n} \cdot \mathbf{n}=1$ and $\mathbf{n} \cdot \mathbf{u}=\Psi_{k}$ with each other. Since both equations are valid along the characteristic surface, its intersection will be valid as well. This is shown in Figure 2 . The equation $\mathbf{n} \cdot \mathbf{n}=1$ simply forms a cylinder of unit radius which is then projected along the $\tau$ axis. The plane $\Psi_{1}=0$ intersects the origin of the coordinate system and its intersection with the unit cylinder forms a cylindrical shape. Since this shape is due to the cylinder constructed by the normal vector $\mathbf{n}$, the surface $\Psi_{1}=0$ is also termed the plane of normals. On each projected normal, within $\Psi_{1}=0$, we can construct a new plane to which the projected normal will be perpendicular. Doing this for an infinite number of normals will create infinite so called stream surfaces, which all intersect in one line. This line corresponds to the pathline of the fluid parcel at that point. The pathline 


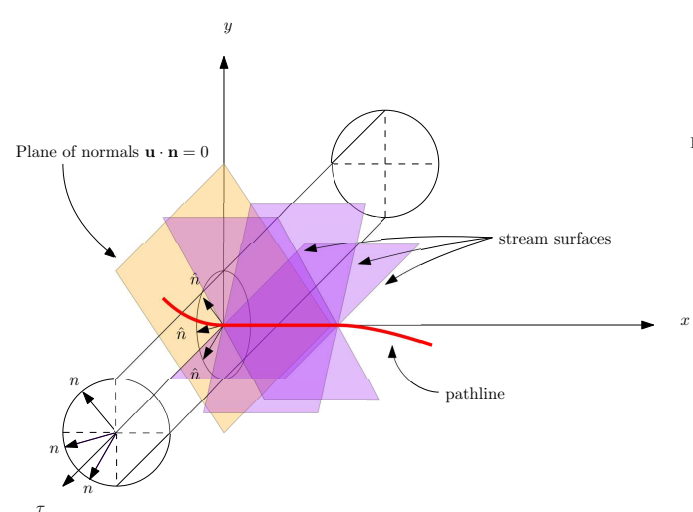

(a) stream surface

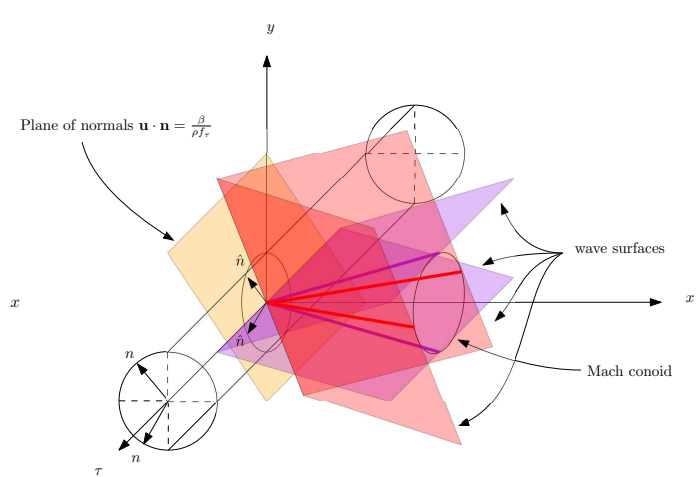

(b) wave surface

Figure 2: Intersection of $\mathbf{n} \cdot \mathbf{n}=1$ and $\mathbf{n} \cdot \mathbf{u}=\Psi_{k}$ and their resulting Stream and Wave surfaces.

satisfies our criterion of $\mathbf{n} \cdot \mathbf{u}=0$ and thus, although reducing to a line, is our first characteristic surface. We can do the same for $\Psi_{2}=\beta /\left(\rho f_{\tau}\right)$, however, the picture becomes slightly more complicated. The plane of normals, again found through the intersections of $\Psi_{2}$ with $\mathbf{n} \cdot \mathbf{n}=1$, does not pass through the origin due to the inhomogeneous term on the right-hand side of $\Psi_{2}$. This is also illustrated in Figure 3, where the unit cylinder spanned by $\mathbf{n} \cdot \mathbf{n}=1$ is shown along with the projected normal vectors $\hat{n}$ onto the plane of normals. The projected unit vectors $\hat{n}$ are normal to the velocity vector $\mathbf{u}$ at their point of intersection. The characteristics emanating from the plane of normals are denoted by dashed lines. Since we have two characteristics emanating from the same point in different directions, we call these lines the bi-characteristics. We construct surfaces from the projected normals inside the plane of normals and call them wave surfaces. Repeating this process for an infinite number of wave surfaces will produce a Mach conoid to which each wave surface will be tangent. It is also sometimes called the pseudo Mach conoid since the Mach conoid analogy is taken from the compressible literature but is absent for incompressible flows. Similarly, we have also adopted the notion of stream and wave surfaces which appear in the literature on compressible CB schemes. Since $\Psi_{2}=\beta /\left(\rho f_{\tau}\right)$ also satisfies $\mathbf{n} \cdot \mathbf{u}=0$, we can use $\Psi_{2}$ as our second characteristic surface. At this point, we need to insert both $\Psi_{1}$ and $\Psi_{2}$ into Eq.(41) and investigate the rank of the coefficient matrix $\mathcal{T}$ arising from the characteristic surfaces. For $\Psi_{1}=0$, we obtain 
Stream surface

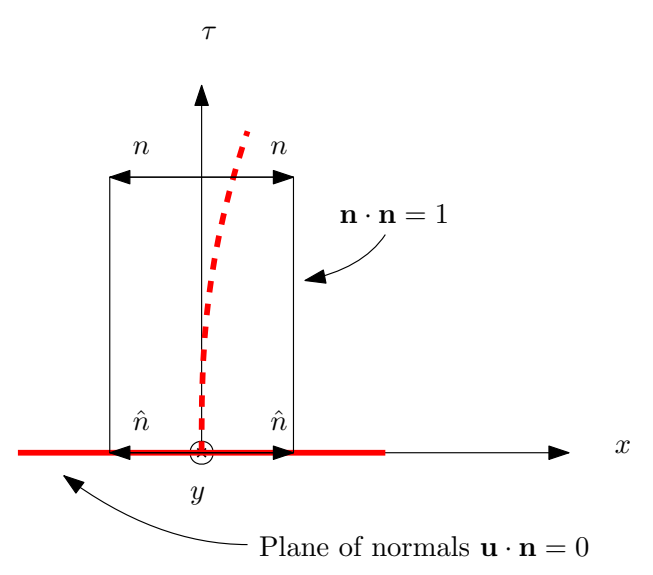

Wave surface

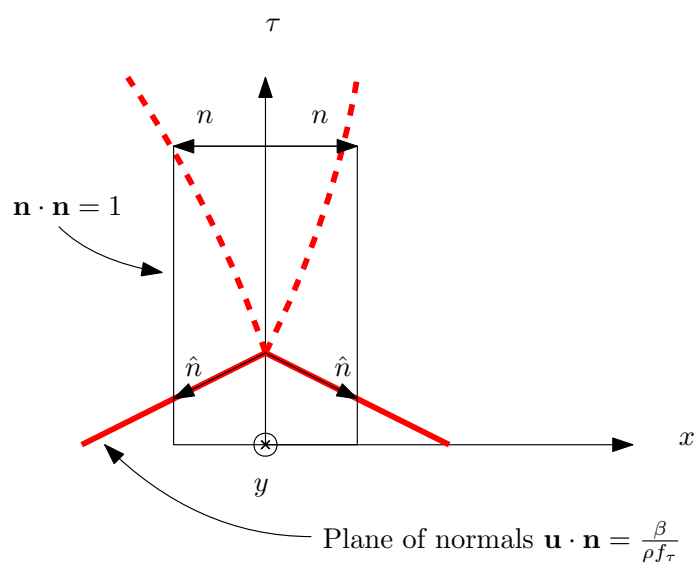

Figure 3: Plane of normals from the $x, \tau$ plane perspective for $\Psi_{1}$ (left) and $\Psi_{2}$ (right).

$$
\left[\begin{array}{ccc}
f_{\tau} / \beta & f_{x} & f_{y} \\
f_{x} / \rho & 0 & 0 \\
f_{y} / \rho & 0 & 0
\end{array}\right]\left[\begin{array}{l}
\mathrm{d} p \\
\mathrm{~d} u \\
\mathrm{~d} v
\end{array}\right]=\left[\begin{array}{l}
0 \\
0 \\
0
\end{array}\right]
$$

To check the rank of the matrix, it is most convenient to express $\mathcal{T}$ in row-echelon form and to count the non-zero rows. We have

$$
\mathcal{T}=\left[\begin{array}{ccc}
\frac{f_{\tau}}{\beta} & f_{x} & f_{y} \\
0 & \frac{-f_{x}^{2} \beta}{\rho f_{\tau}} & \frac{-f_{x} f_{y} \beta}{\rho f_{\tau}} \\
0 & 0 & 0
\end{array}\right]
$$

and so $\operatorname{rank}(\mathcal{T})=2$, which results in $s_{1}=1$, see Eq.(27). Therefore, our coefficient matrix is rank deficient for $\Psi_{1}$ and we cannot obtain a sufficient amount of information along the pathline to construct our compatibility equations. Repeating the process for $\Psi_{2}=\beta /\left(\rho f_{\tau}\right)$, we obtain from Eq.(41)

$$
\left[\begin{array}{ccc}
f_{\tau} / \beta & f_{x} & f_{y} \\
f_{x} / \rho & \frac{\beta}{\rho f_{\tau}} & 0 \\
f_{y} / \rho & 0 & \frac{\beta}{\rho f_{\tau}}
\end{array}\right]\left[\begin{array}{l}
\mathrm{d} p \\
\mathrm{~d} u \\
\mathrm{~d} v
\end{array}\right]=\left[\begin{array}{l}
0 \\
0 \\
0
\end{array}\right] .
$$

The corresponding row-echelon form for $\mathcal{T}$ can be found as 


$$
\mathcal{A}=\left[\begin{array}{ccc}
f_{\tau} & f_{x} & f_{y} \\
0 & \frac{-\beta\left(f_{x}^{2}-1\right)}{\rho f_{\tau}} & \frac{-\beta f_{x} f_{y}}{\rho f_{\tau}} \\
0 & 0 & \frac{\beta\left(f_{x}^{2}+f_{y}^{2}-1\right)}{\rho f_{\tau}\left(f_{x}^{2}-1\right)}
\end{array}\right]
$$

and so $\operatorname{rank}(\mathcal{T})=3$ and therefore $s_{2}=0$. Thus, along our wave surfaces, we have sufficient information available to construct three linear independent compatibility equations and do not need a combination of compatibility equations along the (pseudo) Mach conoid and pathline as is the case for the compressible CB scheme. The corresponding compatibility equations are found as

$$
\begin{aligned}
\frac{f_{\tau}}{\beta} \mathrm{d} p+f_{x} \mathrm{~d} u+f_{y} \mathrm{~d} v & =0, \\
\frac{f_{x}}{\rho} \mathrm{d} p+\frac{\beta}{\rho f_{\tau}} \mathrm{d} u & =0, \\
\frac{f_{y}}{\rho} \mathrm{d} p+\frac{\beta}{\rho f_{\tau}} \mathrm{d} v & =0 .
\end{aligned}
$$

The characteristic variables are obtained from $\mathrm{d} \phi=\phi^{*}-\phi^{n}$, where the asterisk denotes a characteristic variable. We use the normal vector given by Razavi et al. [29] $\mathbf{n}=\cos \phi \cdot \mathbf{i}+\sin \phi \cdot \mathbf{j}$ in space. The normal direction in time can be obtain from equation Eq.(43) as

$$
\Psi_{2}=n_{\tau}+u n_{x}+v n_{y}=\frac{\beta}{\rho n_{\tau}} .
$$

Eq.(51) results in a quadratic equation for which $n_{\tau}$ can be found as

$$
n_{\tau, 1,2}=\frac{-u \cdot \cos \phi-v \cdot \sin \phi \pm \sqrt{(u \cdot \cos \phi+v \cdot \sin \phi)^{2}+4 \frac{\beta}{\rho}}}{2} .
$$

We obtain two solutions for $n_{\tau}$ because we are searching for it on the wave surface which is spanned by the bi-characteristics, i.e. by two independent characteristic paths. We simply choose the positive square root in accordance with the compressible $\mathrm{CB}$ scheme literature. Inserting the normal vector into Eqs.(48)-(50) for the surface derivatives, we obtain 


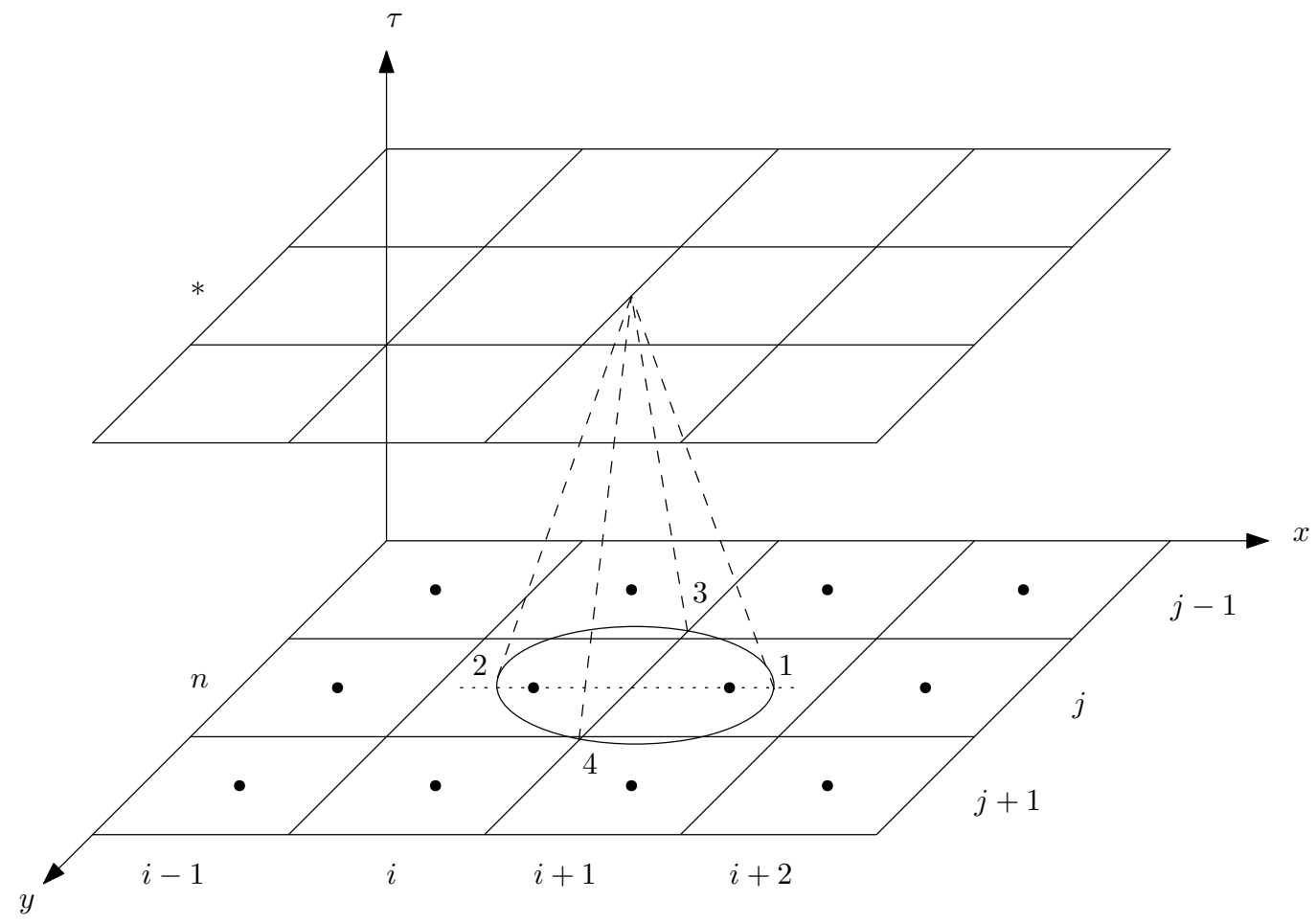

Figure 4: The time discretisation procedure for the multi-directional approach. The pseudo Mach cone intersects the time level $n$ where the four characteristic lines are found $(1,2,3$ and 4). Characteristic line 1 and 2 lie on the intersection of the control volume centroid at $i, j$ and $i+1, j$ while the characteristic lines 3 and 4 emanate from the interfaces at $i+1 / 2$.

$$
\begin{aligned}
& \frac{n_{\tau}}{\beta}\left(p_{i}^{*}-p_{j}^{n}\right)+\left(u_{i}^{*}-u_{j}^{n}\right) \cos \phi+\left(v_{i}^{*}-v_{j}^{n}\right) \sin \phi=0, \\
& \frac{\cos \phi}{\rho}\left(p_{i}^{*}-p_{j}^{n}\right)+\frac{\beta}{\rho n_{\tau}}\left(u_{i}^{*}-u_{j}^{n}\right)=0, \\
& \frac{\sin \phi}{\rho}\left(p_{i}^{*}-p_{j}^{n}\right)+\frac{\beta}{\rho n_{\tau}}\left(v_{i}^{*}-v_{j}^{n}\right)=0 .
\end{aligned}
$$

We have to choose how many wave directions $j$ we want to use along which we update our primitive variables using the compatibility equations. It is customary to choose four independent directions as shown in Figure 4 for a structured hexahedra mesh. Then, for each cell interface, the values at time level $n$ are found at locations $j=1,2,3,4$ which are then used for 
the compatibility equations. If we define $\phi$ in Eqs.(53)-(55) to be measured in anti-clockwise direction with respect to the $x$-axis, then we can find the following compatibility equations at the east inter-cell face $(i+1 / 2)$ :

For $\phi=0^{\circ}$ :

$$
\begin{aligned}
& \frac{n_{\tau}}{\beta}\left(p_{i+1 / 2}^{*}-p_{1}^{n}\right)+\left(u_{i+1 / 2}^{*}-u_{1}^{n}\right)=0 \\
& \left(p_{i+1 / 2}^{*}-p_{1}^{n}\right)+\frac{\beta}{n_{\tau}}\left(u_{i+1 / 2}^{*}-u_{1}^{n}\right)=0 .
\end{aligned}
$$

For $\phi=90^{\circ}$ :

$$
\begin{aligned}
& \frac{n_{\tau}}{\beta}\left(p_{i+1 / 2}^{*}-p_{3}^{n}\right)+\left(v_{i+1 / 2}^{*}-v_{3}^{n}\right)=0 \\
& \left(p_{i+1 / 2}^{*}-p_{3}^{n}\right)+\frac{\beta}{n_{\tau}}\left(v_{i+1 / 2}^{*}-v_{3}^{n}\right)=0 .
\end{aligned}
$$

For $\phi=180^{\circ}$ :

$$
\begin{aligned}
\frac{n_{\tau}}{\beta}\left(p_{i+1 / 2}^{*}-p_{2}^{n}\right)-\left(u_{i+1 / 2}^{*}-u_{2}^{n}\right) & =0, \\
-\left(p_{i+1 / 2}^{*}-p_{2}^{n}\right)+\frac{\beta}{n_{\tau}}\left(u_{i+1 / 2}^{*}-u_{2}^{n}\right) & =0 .
\end{aligned}
$$

For $\phi=270^{\circ}$ :

$$
\begin{aligned}
\frac{n_{\tau}}{\beta}\left(p_{i+1 / 2}^{*}-p_{4}^{n}\right)-\left(v_{i+1 / 2}^{*}-v_{4}^{n}\right) & =0, \\
-\left(p_{i+1 / 2}^{*}-p_{4}^{n}\right)+\frac{\beta}{n_{\tau}}\left(v_{i+1 / 2}^{*}-v_{4}^{n}\right) & =0 .
\end{aligned}
$$

We use Eq.(57) and Eq.(61) to find $u_{i}^{*}$ and Eq.(59) and Eq.(63) to find $v_{i}^{*}$ by eliminating $p_{i}^{*}$ from the equations. We thus obtain for the characteristic velocity components 


$$
\begin{aligned}
u_{i+1 / 2}^{*} & =\frac{1}{\beta\left(n_{\tau, 1}+n_{\tau, 2}\right)}\left[\left(p_{1}-p_{2}\right) n_{\tau, 1} n_{\tau, 2}+\left(u_{1} n_{\tau, 2}+u_{2} n_{\tau, 1}\right) \beta\right], \\
v_{i+1 / 2}^{*} & =\frac{1}{\beta\left(n_{\tau, 3}+n_{\tau, 4}\right)}\left[\left(p_{3}-p_{4}\right) n_{\tau, 3} n_{\tau, 4}+\left(v_{3} n_{\tau, 4}+v_{4} n_{\tau, 3}\right) \beta\right],
\end{aligned}
$$

where in Eq.(52), the variables $u$ and $v$ need to be replaced by $u_{j}$ and $v_{j}$ according to $n_{\tau, j}$. The characteristic pressure can be determined from the Eq.(53) for which have

$$
\begin{aligned}
& p_{i+1 / 2}^{*}=p_{1}-\frac{\beta}{n_{\tau, 1}}\left(u_{i+1 / 2}^{*}-u_{1}\right), \\
& p_{i+1 / 2}^{*}=-p_{2}+\frac{\beta}{n_{\tau, 2}}\left(u_{i+1 / 2}^{*}-u_{2}\right), \\
& p_{i+1 / 2}^{*}=p_{3}-\frac{\beta}{n_{\tau, 3}}\left(v_{i+1 / 2}^{*}-v_{3}\right), \\
& p_{i+1 / 2}^{*}=-p_{4}+\frac{\beta}{n_{\tau, 4}}\left(v_{i+1 / 2}^{*}-v_{4}\right) .
\end{aligned}
$$

An average of Eqs.(66)-(69) results in the final characteristic pressure. The above procedure is repeated for each cell interface, in this case for the west $(i-1 / 2)$, north $(j+1 / 2)$ and south $(j-1 / 2)$ cell interface. The values of $\mathbf{U}_{j}, j=1,2,3,4$ are set to the reconstructed inter-cell values, e.g. $\mathbf{U}_{i+1 / 2}$ at the east face.

Eqs.(64)-(69) have been derived for the AC method and a similar procedure can be repeated for the FSAC-PP method. In this work, however, we leave Eqs.(64)-(65) unchanged but set $p_{i+1 / 2}^{*}=0$. Since the underlying equations are based on the full momentum Equations, including the pressure derivative, it may be argued that this is inconsistent with the modified momentum equation of the FSAC-PP method, where the pressure derivative has been removed. It is important to remember, however, that the developed characteristics-based scheme provides only improved inter-cell values which are then used for the flux differentiation of the correct set of governing equations, i.e. the respective momentum equations of the AC and FSAC-PP method. This provides the advantage that the inter-cell values contain a physical mechanism by which the pressure and velocity are coupled through 
the scheme. This approach is not unique to the current work and has already been proposed, tested and validated in [37, 38, 44] using the SCB scheme. The close coupling may assist to delay the onset of the pressure-velocity decoupling which is inherent to some numerical interpolation schemes.

\subsection{The single-directional characteristics-based scheme for the $A C$ and FSAC- PP method}

For reference, we state here the equations used in the SCB scheme introduced by Drikakis et al. [20]. Its derivation may be found in the original

reference and further considerations given to the derivation for the FSAC-PP method can be found in [37].

The characteristic velocities and pressure are given as

$$
\begin{aligned}
& u_{i+1 / 2}^{*}=\tilde{x} R+\tilde{y}\left(u_{0} \tilde{y}-v_{0} \tilde{x}\right), \\
& v_{i+1 / 2}^{*}=\tilde{y} R+\tilde{x}\left(u_{0} \tilde{y}-v_{0} \tilde{x}\right), \\
& p_{i+1 / 2}^{*}=\frac{1}{2 \sqrt{\lambda_{0}^{2}+\beta}}\left(\lambda_{1} k_{2}-\lambda_{2} k_{1}\right),
\end{aligned}
$$

where

$$
\begin{aligned}
R & =\frac{1}{2 \sqrt{\lambda_{0}^{2}+\beta}}\left[\left(p_{1}-p_{2}\right)+\tilde{x}\left(\lambda_{1} u_{1}-\lambda_{2} u_{2}\right)+\tilde{y}\left(\lambda_{1} v_{1}-\lambda_{2} v_{2}\right)\right], \\
k_{1} & =p_{1}+\lambda_{1}\left(u_{1} \tilde{x}+v_{1} \tilde{y}\right), \\
k_{2} & =p_{2}+\lambda_{2}\left(u_{2} \tilde{x}+v_{2} \tilde{y}\right),
\end{aligned}
$$

with the eigenvalues of the system given as

$$
\begin{aligned}
& \lambda_{0}=u \tilde{x}+v \tilde{y} \\
& \lambda_{1}=\lambda_{0}+\sqrt{\lambda_{0}^{2}+\beta} \\
& \lambda_{2}=\lambda_{0}-\sqrt{\lambda_{0}^{2}+\beta} .
\end{aligned}
$$

The values of $\tilde{x}$ and $\tilde{y}$ are set according to the direction in which the scheme is evaluated, i.e. $\tilde{x}=1$ and $\tilde{y}=0$ in the $x$-direction and $\tilde{x}=0$ 
and $\tilde{y}=1$ in the $y$-direction. In Eqs.(70)-(75), the values of the primitive variables $\mathbf{U}_{j}, j=0,1,2$, are obtained through Godunov's Riemann solver as

$$
\mathbf{U}_{j}=\frac{1}{2}\left[\left(1+\operatorname{sign} \lambda_{j}\right) \mathbf{U}_{i+1 / 2}^{L}+\left(1-\operatorname{sign} \lambda_{j}\right) \mathbf{U}_{i+1 / 2}^{R}\right] .
$$

Here, the values of $\mathbf{U}_{i+1 / 2}^{L, R}$ can be obtained through any appropriate reconstruction scheme, for example using Eq.(10). Just as in the MCB scheme, we set $p_{i+1 / 2}^{*}=0$ for the FSAC-PP method. Eq.(79) provides the necessary upwinding and thus stabilises the scheme. This treatment is absent in the MCB scheme and we will show in the next Section how to introduce approximate Riemann solvers into the MCB scheme.

\section{The Riemann problem in the context of characteristics-based schemes}

In this Section, we will briefly show the relation between the Riemann problem and the method of characteristics as they are closely coupled. Characteristics are defined as those lines in the flow along which derivatives may become discontinuous. This is not a requirement but simply a definition. Characteristics can then be placed through space and the primitive variables are updated along them using the compatibility relations. The Riemann problem, on the other hand, is an initial value problem and defined as

$$
q(x, 0)= \begin{cases}q_{L}, & \text { for } x \leq x_{0} \\ q_{R}, & \text { for } x \geq x_{0}\end{cases}
$$

for some location $x_{0}$. The Riemann problem requires a discontinuous jump in the data which is tracked through time. The primitive variables are then updated based on which side of the discontinuity they are found at. This is also shown in Figure 5. Here, the method of characteristics is shown on the left and the Riemann problem on the right, where a discontinuous signal is advected through time. Two snapshots of the signal are presented at $t_{1}$ and $t_{2}$ in the $(x, q(x))$ space. Those snapshots are transferred to a $(x, t)$ space where different characteristics are shown on the left and the evolution of the initial discontinuity on the right. While in the $\mathrm{CB}$ scheme the primitive variables are updated along the characteristics through compatibility equations which are only valid along their respective characteristics, the Riemann problem distinguishes different states into which the solution can fall. A Riemann 
Characteristics

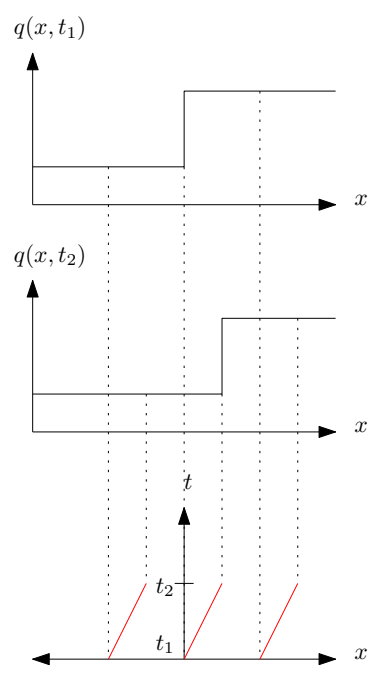

Riemann Solver

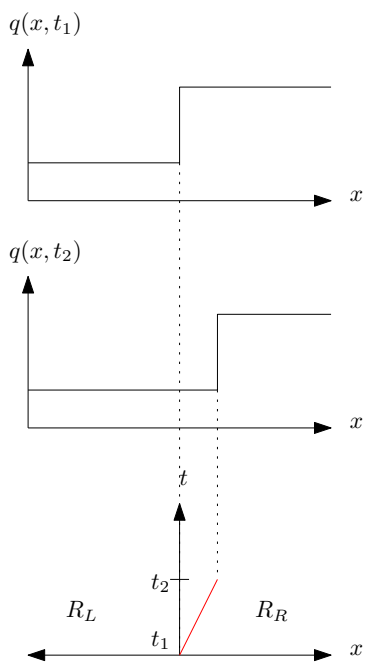

Figure 5: Comaprison of the method of characteristic (left) and the Riemann problem (right) applied to a discontinuous signal tracked in time.

solver would take the information on the discontinuity and make a decision which solution to choose, similar to Eq.(79).

If we define now that the values of the primitive variables prevail over each computational volume, as is the case in a classical finite volume discretisation, then it can be seen from Eq.(10) that at intersecting volumes, discontinuous jumps can occur. Thus, we cannot only use a CB scheme at intersecting volumes but also a Riemann solver or use a combination of both. Note here that using a Riemann solver at each intersecting volume corresponds to the Godunov method [53]. This allows us to make use of the pressure-velocity coupling of the CB scheme while we make use of the decision-making procedure (upwinding) of the Riemann solver.

We use the Riemann solver of Rusanov [54] which is given as

$$
\mathbf{F}(\mathbf{U})_{i+1 / 2}^{\text {Rus }}=\frac{1}{2}\left[\mathbf{F}(\mathbf{U})_{R}+\mathbf{F}(\mathbf{U})_{L}\right]-\frac{S^{+}}{2}\left(\mathbf{U}_{R}-\mathbf{U}_{L}\right) .
$$

Here, $\mathbf{F}(\mathbf{U})$ is the inviscid flux and $S^{+}$a signal-velocity which is given by Davis [55] as 


$$
S^{+}=\max \left\{\left|A_{L}^{-}\right|,\left|A_{R}^{-}\right|,\left|A_{L}^{+}\right|,\left|A_{R}^{+}\right|\right\}
$$

where

$$
\begin{aligned}
& A_{L}^{-}=\mathbf{U}_{L}-\sqrt{\mathbf{U}_{L}^{2}+\beta}, \\
& A_{R}^{-}=\mathbf{U}_{R}-\sqrt{\mathbf{U}_{R}^{2}+\beta}, \\
& A_{L}^{+}=\mathbf{U}_{L}+\sqrt{\mathbf{U}_{L}^{2}+\beta}, \\
& A_{R}^{+}=\mathbf{U}_{R}+\sqrt{\mathbf{U}_{R}^{2}+\beta} .
\end{aligned}
$$

We can replace the left and right velocity vector in Eq.(83) with $\mathbf{U}_{L, R}=$ $u_{L, R} \cos \phi+v_{L, R} \sin \phi$ so as to make it compatible with the MCB scheme. Eq.(81) can then also be used on irregular meshes. In order to couple the MCB scheme with the Riemann solver, we apply it twice at the cell interface, once using $\mathbf{U}_{L}$ and a second time using $\mathbf{U}_{R}$. This creates two characteristic inviscid flux vectors $\mathbf{U}_{L}^{*}$ and $\mathbf{U}_{R}^{*}$ which are then used in Eq.(81). The SCB scheme has been found to be sensitive to its input parameters and both $\mathbf{U}_{L}$ and $\mathbf{U}_{R}$ are required to obtain $\mathbf{U}^{*}$. Note that we do not obtain two individual

states for the primitive variables and thus $\mathbf{F}(\mathbf{U})_{i+1 / 2}^{\text {Rus }}=\mathbf{F}(\mathbf{U})_{i+1 / 2}$. This is likely due to the Riemann solver inherent to the scheme itself, which requires information from both the left and right state. An alternative approach would be use characteristic fluxes in the first term of Eq.(81) and the noncharacteristic primitive variables in the second term. However, the focus here is on the MCB scheme and how to extend it with a Riemann solver. For consistency, we keep the treatment the same for the SCB scheme.

\section{Results and Discussion}

In the following Section, we present results for the lid driven cavity, Taylor-Green vortex and backward-facing step problem to elucidate the advantages and disadvantages of the $\mathrm{MCB}$ scheme in comparison to its singledirectional counterpart. We further show results obtained without a characteristic treatment and compare the aforementioned approaches against our proposed hybrid scheme consisting of the MCB scheme and Rusanov's Riemann solver. We use the central scheme with artificial dissipation which 
we abbreviate with $A D$. The polynomial reconstruction scheme without any characteristic treatment is denoted by no $C B$. The single- and multi-directional characteristic-based scheme which are respectively called the $S C B$ and $M C B$ scheme. Finally, the Rusanov Riemann solver with and without the MCB scheme which are referred on the Figures and Tables as Rus and Rus, MCB, respectively.

\subsection{Flow inside a lid-driven cavity at sub-critical Reynolds numbers}

The lid driven cavity flow is a classical problem to benchmark incompressible flow solvers and methods. Razavi et al. [29] used it to validate their MCB scheme in conjunction with the AC method and compared results against reference data provided by Ghia et al. [56]. There are some issues we need to address first before presenting the results. First of all, the simulations done in Razavi et al. [29] were carried out at different CFL numbers, thus making any direct comparison of the convergence rate troublesome. It was argued that both schemes had different maximum allowable CFL numbers and thus each scheme was simulated with its highest possible CFL number. Numerical experiments showed, however, that the differences are minute for our cases. It should be noted, though, that we use a three stage Runge-Kutta method with TVD properties while a four stage Runge-Kutta method was used in [29]. Furthermore, our definition of the time step is slightly different, taking the effects of diffusion into account.

Another issue was highlighted by Erturk [57]. The lid driven cavity problem by itself is inherently three-dimensional, even at moderate Reynolds numbers, featuring three-dimensional flow features such as the Taylor-Görtler vortices. Thus, a two-dimensional simulation is a purely constructed or artificial solution. However, it has been generally accepted as a standard test case for validation purposes. Care needs to be taken at higher Reynolds numbers; as Erturk pointed out, even a two-dimensional flow will bifurcate at a critical Reynolds number and become unsteady. Erturk presented the literature concerned with obtaining the critical Reynolds number for a two-dimensional flow through direct numerical simulations on a fine grid which suggested it to be around $7000<R e_{\text {crit }}<8000$. In-fact, we found the flow to be periodic and unsteady in this regime and only show results up to $R e=5000$. Razavi et al. [29] showed converged results up to $R e=10000$, which is consistent with the reference data provided by Ghia et al. [56]. It can be argued here that the central scheme with artificial dissipation, which was used in their work, might be used to control the amount of numerical dissipation needed 
in order to achieve a steady state solution. This, however, has to be regarded as a numerical approach to force a steady state solution which might not exist. On the other hand, Erturk showed that by using an increasingly fine mesh, much finer than the reference data considered, that converged results to a steady state are possible even at high Reynolds numbers. Results at $R e=10000$ with a $1024^{2}$ mesh were presented and a steady state solution obtained. The steady state was achieved by decreasing the mesh size, which was adding physical dissipation into the solution. Furthermore, the two-dimensional nature of the problem prohibited the stretching of vortices into the third coordinate direction while inherently three-dimensional flow structures were not able to develop, thus delaying the onset of an unsteady flow. Thus, it may be argued that the steady state achieved in [29] is simply due to the central scheme with artificial dissipation which is adding the dissipation from a numerical point of view which allowed the usage of much coarser meshes. The problem now becomes to determine the right amount of numerical dissipation which matches the physical dissipation without contaminating the overall solution.

Another point of view can be derived by looking at the Taylor series. Consider the truncation of the central differencing scheme with artificial dissipation, Eq.(12), and the third-order polynomial, Eq.(10). In terms of numerical dissipation, we can say that

$$
\nu_{\text {numerical }} \propto \begin{cases}\mathcal{O}\left(\Delta x^{2}\right)+f\left(k_{2}, k_{4}\right), & E q .(12) \\ \mathcal{O}\left(\Delta x^{3}\right), & E q .(10)\end{cases}
$$

where $f\left(k_{2}, k_{4}\right)$ is the influence of the second and forth order derivatives scaled by $k_{2}$ and $k_{4}$. Thus, the amount of dissipation can be controlled, which is not the case for the polynomial interpolation, Eq.(10).

The last issue we need to address is the central scheme itself in conjunction with the Riemann problem. From Eq.(12), we see that it is not possible to construct two individual left- and right-sided interpolated values at the cell interface as for example with Eq.(10). The Riemann problem, however, is defined as an initial value problem with a discontinuity present. We can see from Rusanov's Riemann solver, Eq.(81), that if the left- and right-sided interpolated values at the cell interfaces are equal, the inviscid flux treatment would be independent of the Riemann solver, i.e. in case of the Rusanov Riemann solver we have $\mathbf{F}(\mathbf{U})_{i+1 / 2}^{\mathrm{Rus}}=\mathbf{F}(\mathbf{U})_{i+1 / 2}$. 
We present results here for $R e=100,400,1000,3200$ using both the SCB and MCB scheme along with Rusanov's Riemann solver where applicable and compare the results against a non-characteristic treatment. For all simulations, we use Eq.(10) to interpolate values to the cell interfaces but also give results obtained with central differencing scheme with artificial dissipation (AD), Eq.(12), as a reference. The residuals were calculated based on the divergence of the velocity field and it has been found that a convergence parameter of $\varepsilon=10^{-8}$ has been sufficient to judge convergence. The residuals were normalised after the first iteration to ensure that the solution dropped eight orders of magnitude.

Figure 6 and 7 show the contour plots of the normalised pressure at $\mathrm{Re}=1000$ for the AC and FSAC-PP method, respectively. Looking at the AC method, it can be seen that the pressure exhibits an oscillatory behaviour for the central scheme, non-CB and MCB scheme. What all these schemes have in common is their lack of transportiveness. The central scheme by default cannot distinguish between different flow directions and thus oscillations start to appear at high Reynolds numbers. This problem is well documented in the literature and resulted in the Peclet number restriction when using the central scheme. In the current form, we apply the central scheme with artificial dissipation which aims to compensate for the neglected physical dissipation. This may have a positive impact on developing the velocity field; the pressure field, however, may still show oscillatory behaviour as the lack of transportiveness is not addressed by the artificial dissipation itself. The non-CB and MCB scheme, on the other hand, experience their oscillations in pressure due to the lack of decision-making. The polynomial reconstruction scheme by itself does not provide inbuilt transportiveness, however, it provides left- and right-sided interpolated values. These are the required ingredients to construct transportiveness through some form of decision-making. The simplest form of decision-making is achieved by using an upwind scheme. This scheme simply considers the direction of the flow and sets the left- and right-sided interpolated values according to the flow direction. A more sophisticated approach is presented by employing a Riemann solver which is the approach adopted in the current work. The Riemann solver makes decision about the flow and its direction just as the upwind scheme. It extracts, however, information about the flow through the local eigenstructure of the system. These provide the required transportiveness and it can be seen that the SCB scheme (which features a Riemann solver by default), the Rusanov 


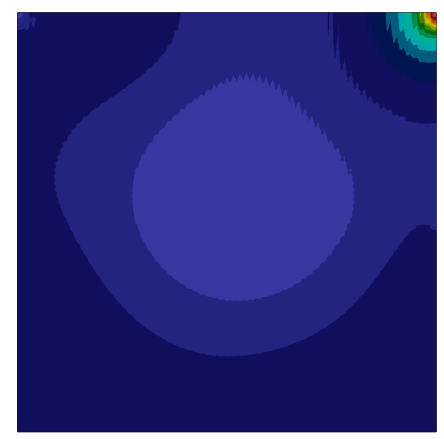

(a) central scheme

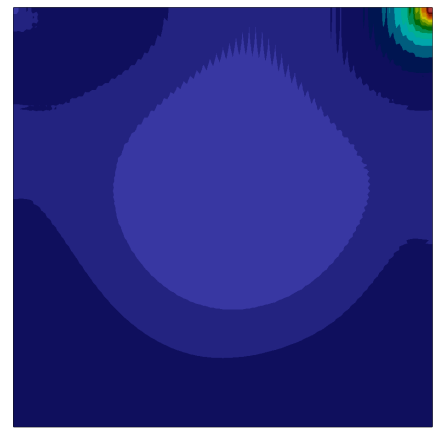

(d) MCB scheme

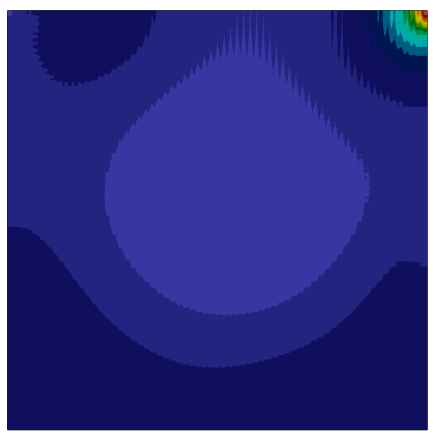

(b) no CB

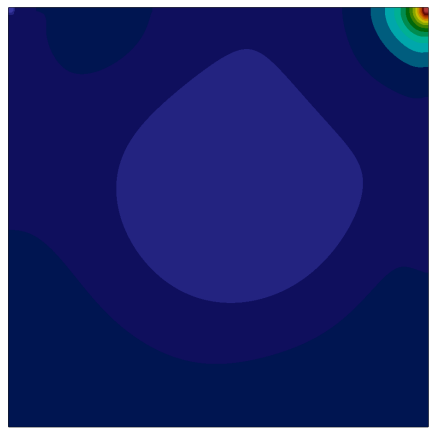

(e) Rusanov RS

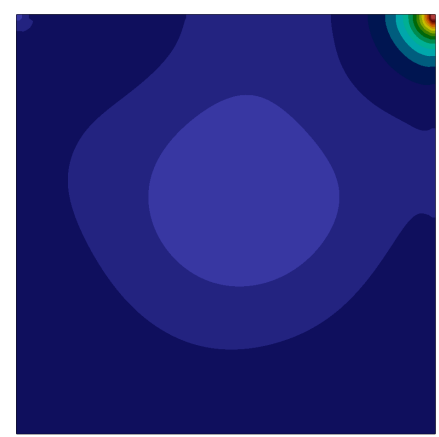

(c) SCB scheme

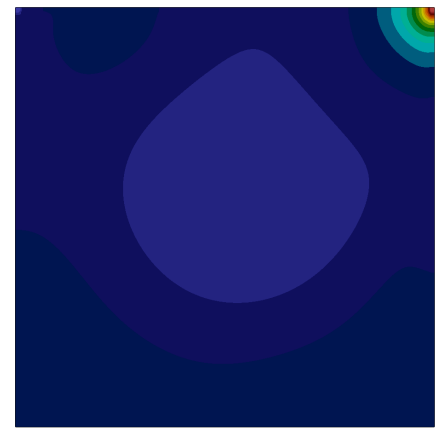

(f) Rusanov RS + MCB

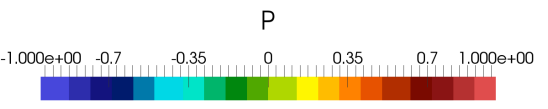

Figure 6: Contour plots of the normalised pressure for the lid driven cavity problem at $\mathrm{Re}=1000$. The result were obtained using the AC method.

Riemann solver, as well as its hybridisation with the MCB scheme all provide smooth pressure contours in Figure 6. Thus, the Riemann solver by itself provides the required transportiveness which is a feature commonly associated to the numerical reconstruction scheme. By using a relative simple Riemann solver, however, we can use a cheap but higher-order interpolation scheme. This scheme may not need to account for any numerical dissipation, as it is the case with the central scheme. On the contrary, the order of the scheme can be increased which decreases the inherent numerical dissipation, based on considerations derived from the Taylor series. Thus, a low-dissipative scheme, such as the third-order polynomial scheme, may be 


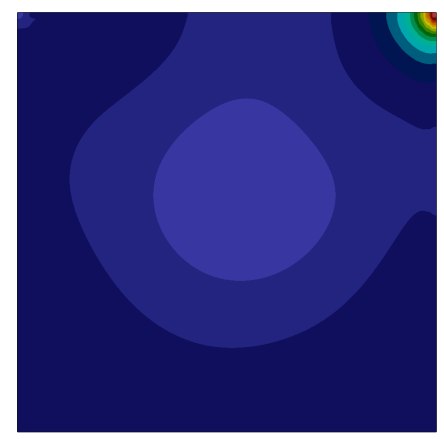

(a) central scheme

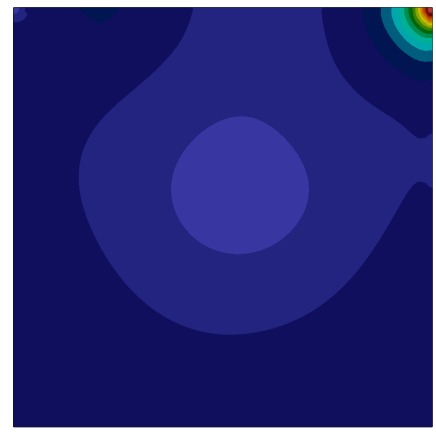

(d) MCB scheme

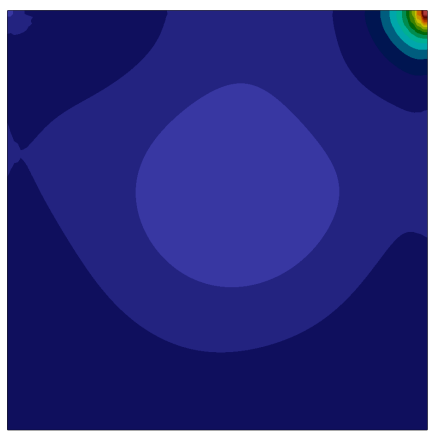

(b) no CB

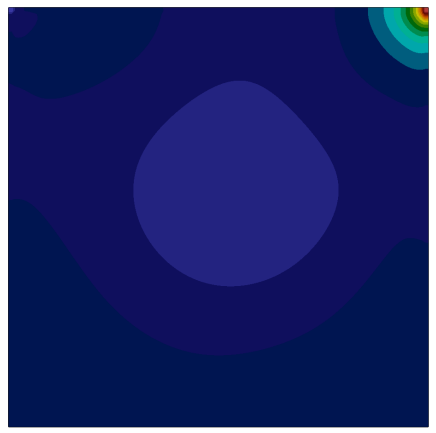

(e) Rusanov RS

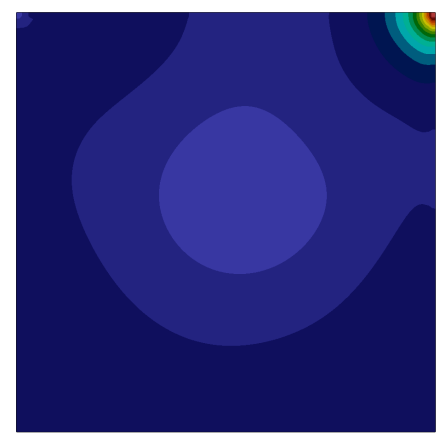

(c) SCB scheme

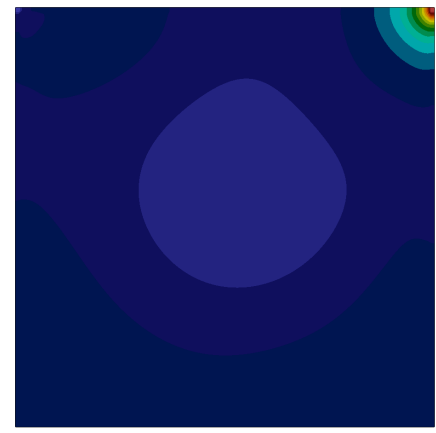

(f) Rusanov RS + MCB

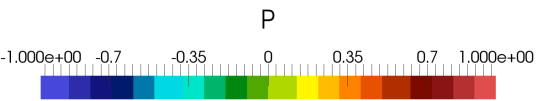

Figure 7: Contour plots of the normalised pressure for the lid driven cavity problem at $\mathrm{Re}=1000$. The result were obtained using the FSAC-PP method.

selected and used in conjunction with the MCB and Rusanov Riemann solver. In this arrangement, the polynomial scheme provides the order of interpolation, the MCB scheme a multi-directional treatment of the flow and the Rusanov Riemann solver the required transportiveness and some numerical dissipation. Overall, the combination of these three components presents a stable and physical approach to treat the non-linear term, while also removing the pressure-velocity decoupling tendencies as commonly found in incompressible flows. Interestingly, the FSAC-PP method shown in Figure 7 does not show signs of any oscillations. This is not merely explained by the numerical schemes employed in this study but is rooted in the philosophy 

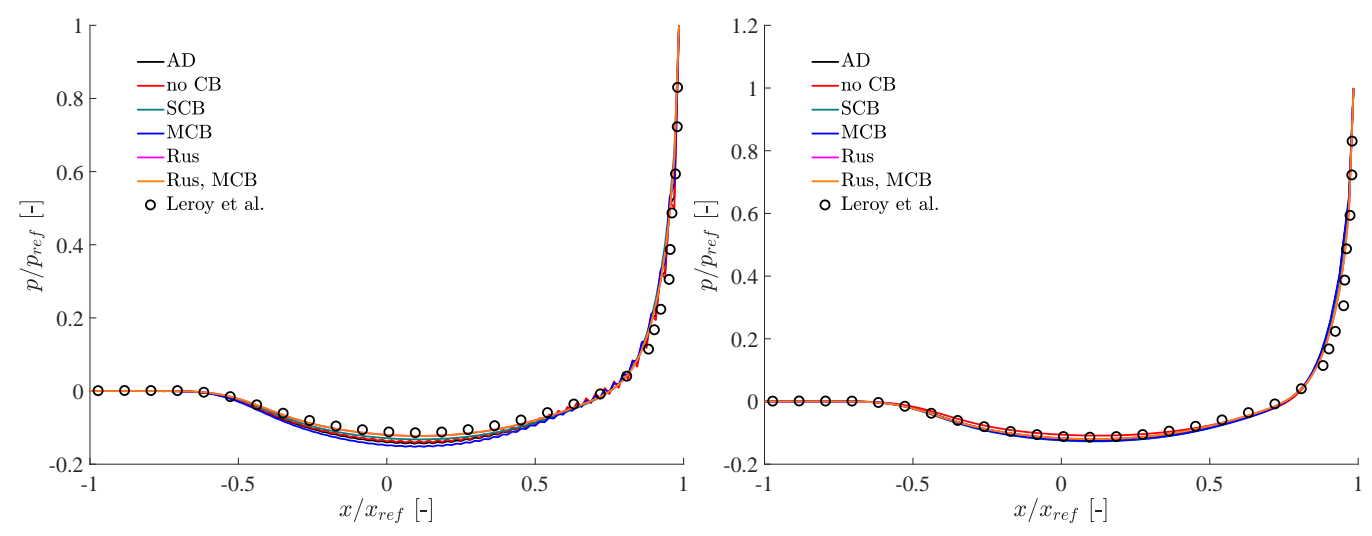

(a) $\mathrm{AC}, \mathrm{Re}=1000$

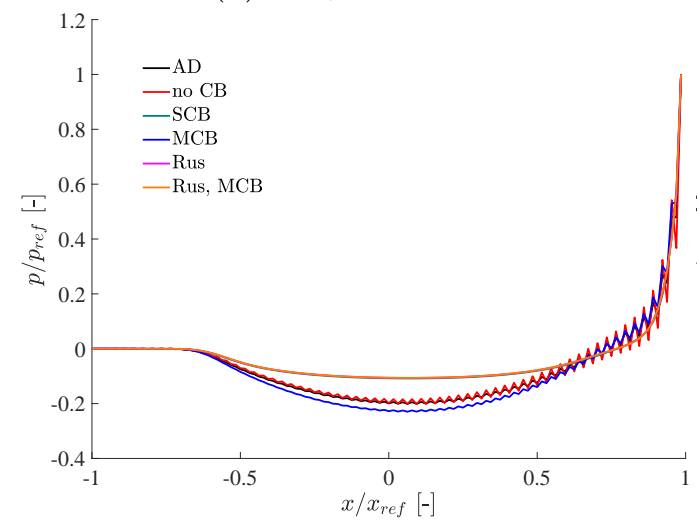

(b) FSAC-PP, Re $=1000$

(c) $\mathrm{AC}, \mathrm{Re}=3200$

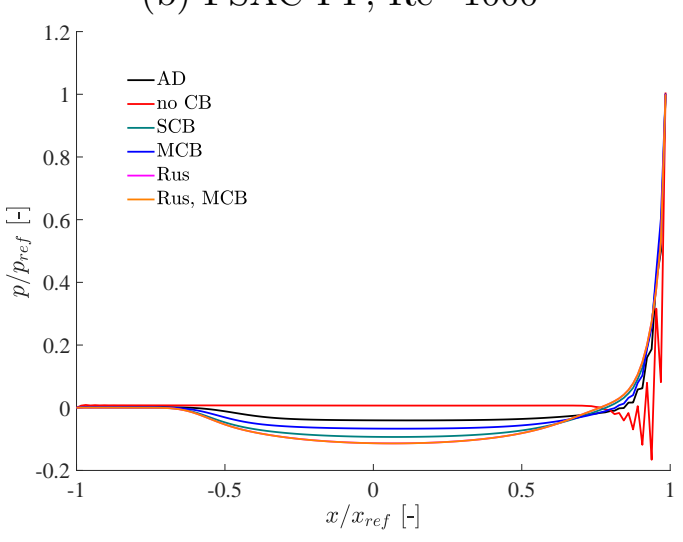

(d) FSAC-PP, Re $=3200$

Figure 8: Pressure profile along the diagonal from the bottom left to top right of the lid driven cavity for different methods at $R e=1000$. The reference data is taken from Leroy et al. [58].

of the FSAC-PP method itself. It features two Fractional-Steps, of which the first one is hyperbolic, allowing the application of the characteristicsbased schemes and Riemann solvers. The second Fractional-Step, however, is purely elliptic which implicitly solves a Pressure Poisson equation. The implicit and elliptic nature have stabilising and smoothing properties which the pressure field benefits from. Any growing oscillations can be damped to a certain degree. This shows that different incompressible methods based on different mathematical characteristics can have a significant influence on the results as well, not just the overall accuracy and convergence rate.

Figure 8 presents the same situation in form of pressure profiles along the 
diagonal of the cavity. In this case, Leroy et al. [58] have provided reference data at $R e=1000$ using the widely validated open-source solver code saturne which we use here to verify our results. We also show results at $R e=3200$ to further highlight some findings. At the reference Reynolds number of $\mathrm{Re}=1000$, we can see that both the AC and FSAC-PP method match those of Leroy et al. [58]. Those oscillations observed in Figure 6 can also be seen in Figure 8a. Similarly, the absence of oscillations in Figure 7 is reflected in Figure 8b. However, it can be seen at the higher Reynolds number of $\mathrm{Re}=3200$, that the oscillations for the AC method become more severe while some oscillations start to show for the FSAC-PP method. Thus, the latter method did not completely prevent oscillations for this particular case, rather, these are delayed through the elliptic smoothing of the pressure field. However, it is only the non-CB scheme which shows considerable oscillations while all other schemes show no or only a small degree of oscillatory behaviour. This is to be expected, as with increasing Reynolds number, the flow becomes more turbulent which also means that the smallest and largest scale are further separated in wave number space. Since the mesh size has not changed, however, this also means that even more physical dissipation is neglected due to ever decreasing eddy sizes through vortex stretching. Thus, it is expected that the scheme with the lowest inherent numerical dissipation is the first to oscillate as the Reynolds number is increased. Indeed, this can be seen by looking at Figure $8 \mathrm{~d}$.

Table 1 and 2 show results obtained from the AC and FSAC-PP method, respectively. The columns are separated into three parts; the reference solution using the central scheme with artificial dissipation, schemes that do not use the Rusanov Riemann solver (RS) and those who do. Note here that although the SCB scheme is in the column where no Rusanov Riemann solver is used, it still contains its own Riemann solver by design, see Eq.(79). In Table 1, we can see that the MCB scheme generally matches or improves the accuracy over a non-characteristic treatment while the number of iterations are of the same order. This effect increases with increasing Reynolds numbers. The SCB scheme, on the other hand, may reduce the overall number of iterations required while its accuracy deteriorates at higher Reynolds numbers. At $\mathrm{Re}=3200$, for example, its $L_{1}$ norm is about two times higher than the non-CB scheme and three times higher than the MCB scheme. Interestingly, with increasing Reynolds number the MCB scheme is capable to match the results of the central differencing scheme with artificial dissipation, which means that the MCB scheme does not only improve the results com- 
Table 1: $L_{0}$ and $L_{1}$ error norm for the $u$ and $v$ velocity component along the horizontal and vertical centreline, respectively. The results were obtained using the AC method.

\begin{tabular}{|c|c|c|c|c|c|c|c|}
\hline \multirow[b]{2}{*}{$\operatorname{Re}$} & & \multirow{2}{*}{$\frac{\mathrm{AD}}{\text { no } \mathrm{CB}}$} & \multicolumn{3}{|c|}{ no Riemann Solver } & \multicolumn{2}{|c|}{ Rusanov Riemann Solver } \\
\hline & & & no $\mathrm{CB}$ & $\mathrm{SCB}$ & $\mathrm{MCB}$ & no $\mathrm{CB}$ & $\mathrm{MCB}$ \\
\hline \multirow{5}{*}{100} & iteration & 62199 & 62200 & 62114 & 62174 & 60575 & 60575 \\
\hline & $L_{0}(u)[\%]$ & 0.42 & 0.41 & 0.62 & 0.45 & 0.67 & 0.67 \\
\hline & $L_{0}(v)[\%]$ & 0.85 & 0.84 & 0.33 & 0.84 & 0.41 & 0.41 \\
\hline & $L_{1}(u)[\%]$ & 0.14 & 0.14 & 0.23 & 0.15 & 0.25 & 0.25 \\
\hline & $L_{1}(v)[\%]$ & 0.36 & 0.36 & 0.19 & 0.37 & 0.21 & 0.21 \\
\hline \multirow{5}{*}{400} & iteration & 60798 & 60811 & 58711 & 60053 & 56480 & 56480 \\
\hline & $L_{0}(u)[\%]$ & 0.52 & 0.61 & 3.51 & 0.50 & 3.69 & 3.69 \\
\hline & $L_{0}(v)[\%]$ & 4.45 & 4.34 & 3.41 & 4.38 & 3.62 & 3.62 \\
\hline & $L_{1}(u)[\%]$ & 0.20 & 0.25 & 1.38 & 0.20 & 1.38 & 1.38 \\
\hline & $L_{1}(v)[\%]$ & 0.42 & 0.44 & 1.88 & 0.36 & 1.96 & 1.96 \\
\hline \multirow{5}{*}{1000} & iteration & 124467 & 125231 & 115499 & 123685 & 107382 & 107382 \\
\hline & $L_{0}(u)[\%]$ & 1.45 & 1.84 & 6.53 & 1.55 & 6.49 & 6.49 \\
\hline & $L_{0}(v)[\%]$ & 0.69 & 0.99 & 6.39 & 0.62 & 6.50 & 6.50 \\
\hline & $L_{1}(u)[\%]$ & 0.60 & 0.80 & 3.25 & 0.58 & 2.86 & 2.86 \\
\hline & $L_{1}(v)[\%]$ & 0.41 & 0.63 & 3.98 & 0.37 & 3.79 & 3.79 \\
\hline \multirow{5}{*}{3200} & iteration & 226470 & 213636 & 206070 & 225901 & 195489 & 195489 \\
\hline & $L_{0}(u)[\%]$ & 4.92 & 7.99 & 14.75 & 4.90 & 11.92 & 11.92 \\
\hline & $L_{0}(v)[\%]$ & 5.25 & 10.82 & 14.63 & 7.20 & 13.08 & 13.08 \\
\hline & $L_{1}(u)[\%]$ & 2.71 & 4.33 & 8.86 & 2.75 & 6.48 & 6.48 \\
\hline & $L_{1}(v)[\%]$ & 3.15 & 5.26 & 10.66 & 3.37 & 8.34 & 8.34 \\
\hline
\end{tabular}


Table 2: $L_{0}$ and $L_{1}$ error norm for the $u$ and $v$ velocity component along the horizontal and vertical centreline, respectively. The results were obtained using the FSAC-PP method.

\begin{tabular}{|c|c|c|c|c|c|c|c|}
\hline \multirow[b]{2}{*}{$\operatorname{Re}$} & & \multirow{2}{*}{$\frac{\mathrm{AD}}{\text { no } \mathrm{CB}}$} & \multicolumn{3}{|c|}{ no Riemann Solver } & \multicolumn{2}{|c|}{ Rusanov Riemann Solver } \\
\hline & & & no $\mathrm{CB}$ & SCB & $\mathrm{MCB}$ & no $\mathrm{CB}$ & $\mathrm{MCB}$ \\
\hline \multirow{5}{*}{100} & iteration & 12635 & 12628 & 12991 & 10683 & 12815 & 10590 \\
\hline & $L_{0}(u)[\%]$ & 0.55 & 0.57 & 0.49 & 0.72 & 0.52 & 0.71 \\
\hline & $L_{0}(v)[\%]$ & 0.27 & 0.29 & 0.27 & 0.44 & 0.23 & 0.44 \\
\hline & $L_{1}(u)[\%]$ & 0.19 & 0.20 & 0.19 & 0.24 & 0.19 & 0.24 \\
\hline & $L_{1}(v)[\%]$ & 0.18 & 0.18 & 0.16 & 0.22 & 0.16 & 0.22 \\
\hline \multirow{5}{*}{400} & iteration & 17281 & 17322 & 16544 & 16593 & 15689 & 15810 \\
\hline & $L_{0}(u)[\%]$ & 3.71 & 4.17 & 3.49 & 3.84 & 3.39 & 3.33 \\
\hline & $L_{0}(v)[\%]$ & 3.89 & 4.36 & 3.52 & 3.88 & 3.32 & 3.28 \\
\hline & $L_{1}(u)[\%]$ & 1.55 & 1.74 & 1.42 & 1.55 & 1.30 & 1.28 \\
\hline & $L_{1}(v)[\%]$ & 2.08 & 2.29 & 1.92 & 2.09 & 1.83 & 1.81 \\
\hline \multirow{5}{*}{1000} & iteration & 32567 & 36835 & 28232 & 33852 & 24747 & 24141 \\
\hline & $L_{0}(u)[\%]$ & 7.81 & 10.98 & 6.33 & 7.75 & 5.23 & 5.20 \\
\hline & $L_{0}(v)[\%]$ & 8.16 & 11.50 & 6.86 & 8.40 & 5.77 & 5.71 \\
\hline & $L_{1}(u)[\%]$ & 4.27 & 5.80 & 3.37 & 4.10 & 2.40 & 2.38 \\
\hline & $L_{1}(v)[\%]$ & 5.32 & 7.35 & 4.16 & 5.13 & 3.20 & 3.17 \\
\hline \multirow{5}{*}{3200} & iteration & 134019 & 40826 & 128379 & 133148 & 63737 & 65701 \\
\hline & $L_{0}(u)[\%]$ & 29.08 & 41.78 & 16.49 & 23.09 & 9.67 & 9.63 \\
\hline & $L_{0}(v)[\%]$ & 33.91 & 54.42 & 17.04 & 25.46 & 11.28 & 11.19 \\
\hline & $L_{1}(u)[\%]$ & 17.46 & 24.56 & 9.90 & 13.88 & 4.97 & 4.93 \\
\hline & $L_{1}(v)[\%]$ & 21.79 & 32.85 & 12.08 & 17.15 & 6.65 & 6.60 \\
\hline
\end{tabular}


pared to a non-CB scheme, which uses the same, low-dissipative third-order polynomial to interpolated inter-cell values, but also that the added dissipation in the central scheme provides the same level of accuracy as the MCB scheme. Thus, although the underlying interpolation scheme struggles to provide accurate results due to the neglected dissipation, the MCB scheme is able to compensate for that fact and shows no clear differences to the central scheme which relies on numerical dissipation. Referring back to Figure 8c, it can be seen that the MCB scheme also suffers from the velocity decoupling as discussed above due to the absence of transportiveness in the overall scheme, however, it exhibits less oscillations than the non-CB scheme. It has also been stated that the Rusanov Riemann solver is adding more numerical dissipation along with transportiveness to the scheme, which ultimately results in oscillation free pressure profiles. It can also be seen from Table 1 that the Rusanov Riemann solver is indeed adding numerical dissipation, in the sense that the results become less accurate. Measuring the numerical dissipation is inherently difficult and we restrict the discussion here on the symptoms such as reduced accuracy and increased oscillatory behaviour. It should be noted, however, that first promising results were published by El Rafei et al. [59] who computed the numerical dissipation directly through the modified equation analysis for the complete set of Navier-Stokes equations. Despite the reduced accuracy, the number of iterations were reduced for some cases up to $14 \%$ compared to a non Rusanov Riemann solver treatment. Furthermore, it was shown that the SCB scheme is less accurate than the MCB scheme at higher Reynolds numbers. However, it was also shown that the SCB scheme provides oscillation free pressure profiles even at higher Reynolds numbers. The same can be achieved for the MCB scheme when it is paired with the Rusanov Riemann solver, as can be seen from Figure 8c and $8 \mathrm{~d}$. The fact that at lower Reynolds numbers little differences can be observed is explained by the laminar nature of the flow. If the flow is laminar, the scale separation of smallest and largest eddies does not exist due to a lack of turbulence in the flow. Thus, all dissipation is captured by the Laplacian term in the Navier-Stokes equations and therefore fully resolved. Therefore, it is the higher Reynolds numbers which are of interest to judge the capabilities of the proposed schemes. Interestingly, there is no difference between using just the Rusanov Riemann solver or its combination with the MCB scheme, which is unique to the AC method.

In Table 2, the same results are shown for the FSAC-PP method. It is apparent that it converges faster than the $\mathrm{AC}$ method. At $R e=100$, it is 
up to 5.8 times faster compared to the AC method (MCB scheme) while at higher Reynolds numbers, here at $R e=3200$, a speed-up of a factor of 1.7-3.1 can be still achieved. This is in accordance with previously published material [37, 38, 44, 45], where the FSAC-PP method has generally been found to perform better over the $\mathrm{AC}$ method in terms of convergence rate. It could be argued that the inclusion of the Poisson solver slows the solution procedure down, however, numerical experiments have shown that the AC method is only about 1.5 times faster than the FSAC-PP method per iteration. Thus, the FSAC-PP method requires overall less CPU time for all Reynolds numbers considered here. At $\mathrm{Re}=3200$, it becomes clear that results without a Riemann solver lose the accuracy which was also seen by Figure 8d, where the non-CB scheme seemingly did not develop any pressure field. Even the added dissipation approach starts to lose accuracy, showing that numerical dissipation alone is not enough to provide physical results, if it is not properly scaled with the required dissipation. Thus, the added transportiveness of the Riemann solver makes the biggest difference which results in more accurate results at a reduced computational cost. In contrast to the AC method, the SCB scheme is slightly more accurate than the MCB scheme. This complete reversal shows the importance of the choice of the incompressible method which is not merely responsible for the required number of iterations. Its mathematical character plays an important role in how the underlying numerical schemes perform.

Figure 9 shows the velocity profiles for the AC and FSAC-PP method on the centreline at $R e=5000$ using a $128^{2}$ and $256^{2}$ mesh. As was already remarked when discussing Table 1 for the AC method, there is no difference between the Rusanov Riemann solver approach and its hybrid version with the MCB scheme combined. The same is true at $\mathrm{Re}=5000$ and the curves for both schemes are indistinguishable. Examining Figure 9a and 9b, it can be seen that on a $128^{2}$ mesh, the dissipation provided is not sufficient to develop the flow using a non-CB treatment. The Riemann solver in the SCB scheme provides enough dissipation for the flow to develop and similar observations are made for the Rusanov Riemann solver approach. It does indeed seem that the numerical dissipation provided by the Riemann solvers is sufficient at higher Reynolds numbers to correctly develop the flow, despite the low-dissipative polynomial reconstruction scheme. This approach is quite similar to the philosophy of implicit large eddy simulations, where the sub-grid scale model is replaced and modelled through numerical dissipation provided through the numerical interpolation schemes. Alternatively, 


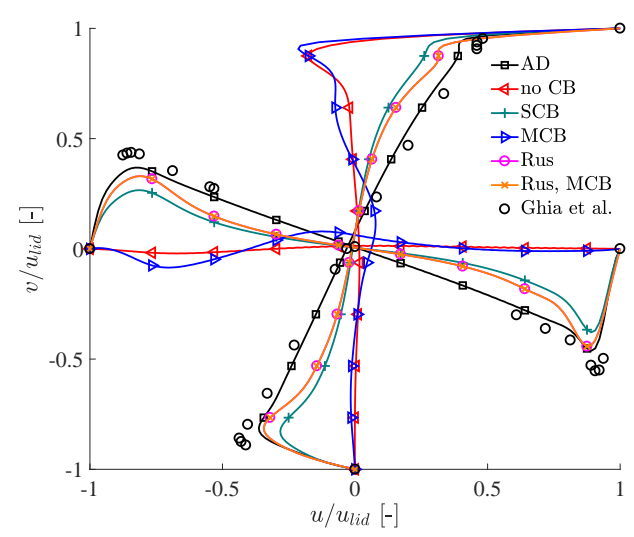

(a) AC, $128^{2}$ cells

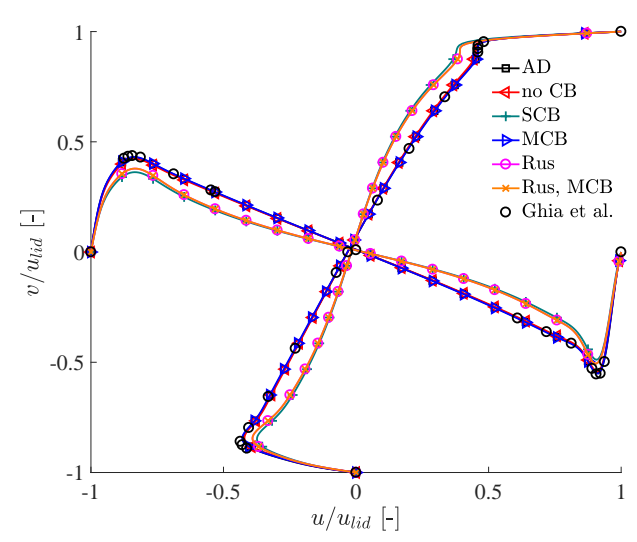

(c) AC, $256^{2}$ cells

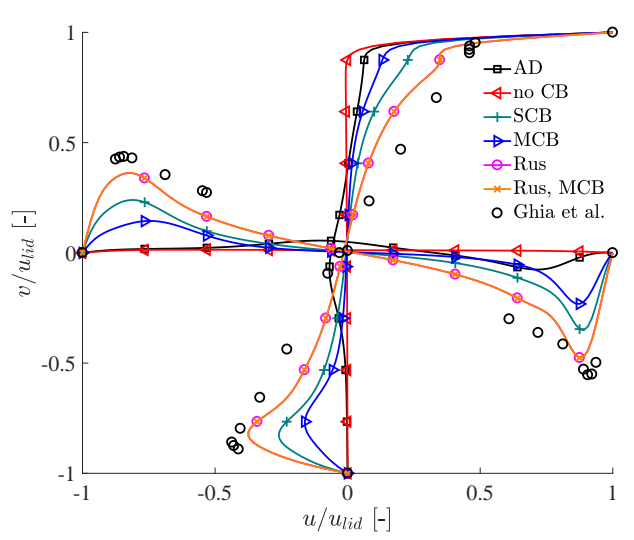

(b) FSAC-PP, $128^{2}$ cells

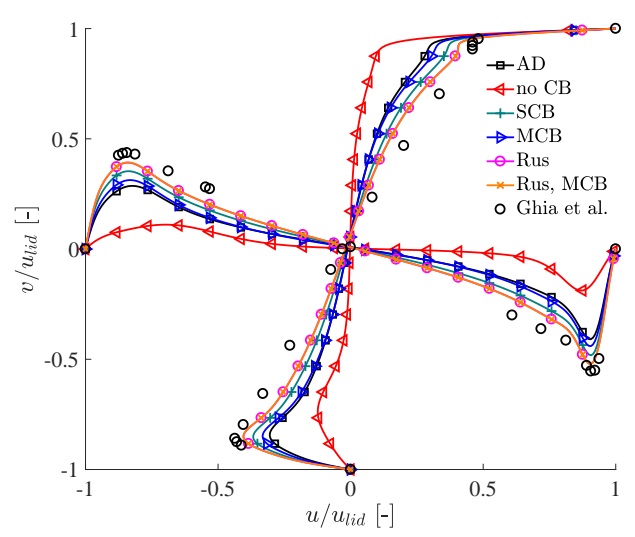

(d) FSAC-PP, 256 cells

Figure 9: Velocity profiles along the centrelines of the lid driven cavity flow problem at $\mathrm{Re}=5000$ using $128^{2}$ and $256^{2}$ cells and the AC and FSAC-PP method. The reference data is taken from Ghia et al. [56].

the mesh size can be increased which results in more physical dissipation being recovered and resolved. This approach is similar to the one taken by Erturk [57] in order to obtain converged, two-dimensional results using here a low-dissipative scheme. The results are shown for the AC and FSAC-PP method in Figure 9c and Figure 9d, respectively. Using the AC method, the Riemann solver increases the overall accuracy again while it approaches the solution of the SCB scheme. In the case of the FSAC-PP method, the overall accuracy is increased and the method generally reacts more favourably to a 
Table 3: $L_{0}$ and $L_{1}$ norm for the lid driven cavity problem at $\mathrm{Re}=5000$ on a $128^{2}$ and $256^{2}$ mesh. Results shown were obtained with the AC method.

\begin{tabular}{|c|c|c|c|c|c|c|c|}
\hline \multirow[b]{2}{*}{$\left(n_{x} \times n_{y}\right)$} & & \multirow{2}{*}{$\frac{\mathrm{AD}}{\mathrm{no} \mathrm{CB}}$} & \multicolumn{3}{|c|}{ no Riemann Solver } & \multicolumn{2}{|c|}{ Rusanov Riemann Solver } \\
\hline & & & no $\mathrm{CB}$ & $\mathrm{SCB}$ & $\mathrm{MCB}$ & no $\mathrm{CB}$ & $\mathrm{MCB}$ \\
\hline \multirow{5}{*}{$128^{2}$} & iteration & 233577 & 299288 & 257593 & 500000 & 221751 & 221751 \\
\hline & $L_{0}(u)[\%]$ & 12.35 & 60.46 & 18.83 & 67.47 & 14.44 & 14.44 \\
\hline & $L_{0}(v)[\%]$ & 16.87 & 55.57 & 22.55 & 54.63 & 19.52 & 19.52 \\
\hline & $L_{1}(u)[\%]$ & 4.84 & 29.76 & 12.07 & 32.05 & 8.38 & 8.38 \\
\hline & $L_{1}(v)[\%]$ & 5.96 & 35.03 & 14.35 & 36.22 & 10.40 & 10.40 \\
\hline \multirow{5}{*}{$256^{2}$} & iteration & 1306388 & 953483 & 480368 & 2434067 & 545401 & 545401 \\
\hline & $L_{0}(u)[\%]$ & 2.07 & 3.05 & 9.28 & 2.07 & 8.39 & 8.39 \\
\hline & $L_{0}(v)[\%]$ & 1.38 & 2.73 & 9.13 & 1.77 & 8.40 & 8.40 \\
\hline & $L_{1}(u)[\%]$ & 0.46 & 0.90 & 5.72 & 0.39 & 4.82 & 4.82 \\
\hline & $L_{1}(v)[\%]$ & 0.43 & 0.95 & 6.43 & 0.37 & 5.39 & 5.39 \\
\hline
\end{tabular}

Riemann solver treatment.

These results are also summarised quantitatively in Table 3 and 4 . For the AC method, the MCB scheme did not converge and was stopped after 500 000 iterations on the $128^{2}$ mesh. The iterations and intermediate solution suggest that an unsteady behaviour was found and that convergence to the steady state was not possible. This is in contrast to the FSAC-PP method where a steady state solution was achieved. At $R e=5000$, we should still be able to obtain a steady state solution according to Erturk [57]. While the AC method only achieved that by increasing the mesh size, thus providing more physical dissipation, the FSAC-PP method was able to smooth the flow field sufficiently through the elliptic Poisson solver, providing the steady state solution. The trends observed in Table 1 and 2 are broadly the same at $R e=5000$ for both mesh sizes. For the AC method, the central scheme with artificial dissipation and the non-CB scheme required substantially more iterations going from the $128^{2}$ to the $256^{2}$ mesh while the accuracy was positively affected, especially in the latter case. The MCB scheme was not only able to find a steady state solution but also improve the overall accuracy, which 
Table 4: $L_{0}$ and $L_{1}$ norm for the lid driven cavity problem at $\mathrm{Re}=5000$ on a $128^{2}$ and $256^{2}$ mesh. Results shown were obtained with the FSAC-PP method.

\begin{tabular}{|c|c|c|c|c|c|c|c|}
\hline \multirow[b]{2}{*}{$\left(n_{x} \times n_{y}\right)$} & & \multirow{2}{*}{$\frac{\mathrm{AD}}{\text { no } \mathrm{CB}}$} & \multicolumn{3}{|c|}{ no Riemann Solver } & \multicolumn{2}{|c|}{ Rusanov Riemann Solver } \\
\hline & & & no $\mathrm{CB}$ & $\mathrm{SCB}$ & $\mathrm{MCB}$ & no $C B$ & $\mathrm{MCB}$ \\
\hline \multirow{5}{*}{$128^{2}$} & iteration & 273280 & 53677 & 203316 & 200288 & 100291 & 105308 \\
\hline & $L_{0}(u)[\%]$ & 43.49 & 44.22 & 22.04 & 31.54 & 11.92 & 11.90 \\
\hline & $L_{0}(v)[\%]$ & 54.56 & 55.91 & 26.14 & 38.15 & 17.56 & 17.48 \\
\hline & $L_{1}(u)[\%]$ & 24.65 & 26.41 & 13.88 & 19.34 & 6.48 & 6.45 \\
\hline & $L_{1}(v)[\%]$ & 32.59 & 34.12 & 16.70 & 23.66 & 8.21 & 8.16 \\
\hline \multirow{5}{*}{$256^{2}$} & iteration & 124962 & 353119 & 156646 & 227812 & 135563 & 136772 \\
\hline & $L_{0}(u)[\%]$ & 16.83 & 34.97 & 10.93 & 15.05 & 7.63 & 7.56 \\
\hline & $L_{0}(v)[\%]$ & 16.65 & 40.98 & 10.61 & 14.98 & 7.65 & 7.56 \\
\hline & $L_{1}(u)[\%]$ & 10.53 & 21.08 & 6.43 & 9.19 & 3.96 & 3.91 \\
\hline & $L_{1}(v)[\%]$ & 12.33 & 25.82 & 7.34 & 10.61 & 4.47 & 4.42 \\
\hline
\end{tabular}

shows the closest agreement with the reference data. The Rusanov-based approach shows higher errors while keeping the number of iterations low on the finer mesh. For the FSAC-PP method, however, the central scheme with artificial dissipation and the SCB scheme both show a reduction in iterations when going from the coarser to the finer mesh, while improving also the accuracy. The best results are now obtained with the Rusanov-based approaches which show a similar level of accuracy but a reduced number of iterations compared to the AC method. In-fact, the FSAC-PP method requires about 4.0 times less iterations than the AC method.

In summary, we showed that the overall agreement of the AC method with the reference data can be slightly better using a non Riemann solver treatment over the FSAC-PP method, while the latter method improved the overall convergence rate. The MCB scheme has shown that it is able to reduce the number of iterations and be more accurate for some cases as was reported in [29]. We could not, however, confirm a speed-up of the MCB scheme over the SCB scheme using the AC method at the same CFL number. Using a 
Riemann solver, both methods showed comparable accuracies. The inclusion of the Riemann solver was found to be necessary at high Reynolds numbers to provide enough numerical dissipation to develop the flow. Alternatively, the central scheme with artificial dissipation can provide a user-defined amount of dissipation that scales with the mesh size. Fine tuning of the scheme's parameter may be necessary which is not the case in the Riemann solver. Furthermore, we saw that the mixed hyperbolic-elliptic behaviour of the FSAC-PP method can produce a quite different behaviour than its purely hyperbolic AC counterpart when comparing the accuracy and iterations required of the SCB and MCB scheme. Thus, not just the numerical scheme but also the incompressible method that is employed to solve the incompressible Navier-Stokes equations plays a crucial role in determining the correct behaviour of the flow.

\subsection{Time-dependent evolution of the Taylor-Green vortex}

As highlighted in the lid driven cavity flow problem, the numerical dissipation is a crucial element in determining a scheme's success. It is especially important for the MCB scheme as it does not provide a Riemann solver by default, which is included in the SCB scheme. San and Kara [60] investigated several Riemann solvers and showed that the Rusanov Riemann solver shows indeed higher or similar numerical damping compared to other Riemann solvers. In this Section, we investigate the Taylor-Green vortex problem, where we have an analytic solution available for the velocity and pressure field. Thus, we can calculate the overall kinetic energy and compare it to the simulations. An increased rate of dissipation of the kinetic energy implies and increased amount of numerical dissipation. Since it was established in the previous example that at high Reynolds number, i.e. within the turbulent regime, the MCB is benefiting from the added dissipation from the Rusanov Riemann solver, we present results in the present case for Reynolds numbers up to $R e=1000$. It has been stated by Drikakis et al. [61], that results obtained at $R e=3000$ and $R e=5000$ are almost indistinguishable, indicating a low viscosity limit. At the same time, we found that a larger time interval needed to be considered when increasing the Reynolds number and thus decided that $R e=1000$ was best suited to strike a balance between computational time and flow physics. Although we consider a twodimensional flow in this work, Brachet et al. [62] performed two-dimensional DNS studies and showed that essential turbulent-like features were captured for this particular flow type. We also perform simulations at $\mathrm{Re}=100$ and 
$\mathrm{Re}=500$ to have a reference solution for a fully laminar flow and one between the laminar and turbulent regime, to highlight any change in the behaviour of the numerical schemes in these two states. Since the Taylor-Green vortex is an evolution problem, we need to consider the unsteady version of the AC and FSAC-PP method. In the previous literature on the MCB scheme, only steady state problems were considered and so we are able to comment on the scheme's behaviour for unsteady flows for the first time. We reduce the convergence parameter to $\varepsilon=10^{-4}$ within each pseudo time step and integrate the solution until we have reached $t=15, t=30$ and $t=50$ seconds at $R e=100, R e=500$ and $R e=1000$, respectively, of simulated flow time.

The flow is solved on a square computational domain with periodic boundary conditions on all sides. Due to the added computational cost of solving the equations in time, we use a $64^{2}$ mesh for all simulations. The flow field is initialised with

$$
\begin{aligned}
& u_{0}(x, y, 0)=-\cos (2 m \pi x) \sin (2 m \pi y), \\
& v_{0}(x, y, 0)=\sin (2 m \pi x) \cos (2 m \pi y), \\
& p_{0}(x, y, 0)=-\frac{1}{4}[\cos (4 m \pi x)+\cos (4 m \pi y)],
\end{aligned}
$$

for which an analytical solution with $\nu \neq 0$ can be found as [47]

$$
\begin{aligned}
& u(x, y, t)=u_{0}(x, y, 0) \exp \left[-2 / \operatorname{Re}(2 m \pi)^{2} t\right], \\
& v(x, y, t)=v_{0}(x, y, 0) \exp \left[-2 / \operatorname{Re}(2 m \pi)^{2} t\right], \\
& p(x, y, t)=p_{0}(x, y, 0) \exp \left[-4 / \operatorname{Re}(2 m \pi)^{2} t\right] .
\end{aligned}
$$

For $R e \rightarrow \infty$, or $\nu \rightarrow 0$, an inviscid limit is approached as stated by Drikakis et al. [61]. We can see from Eq.(86) that indeed the solution approaches an inviscid limit for a vanishing viscosity. Here, we set $m=2$ where higher values would result in more but smaller vortices. The initial velocity and pressure contours are given in Figure 10 where the streamlines are given as a reference to highlight the symmetrical initial vortex distribution.

Figure 11 shows the decay of kinetic energy for both the AC and FSACPP method across different Reynolds numbers which is compared against the 


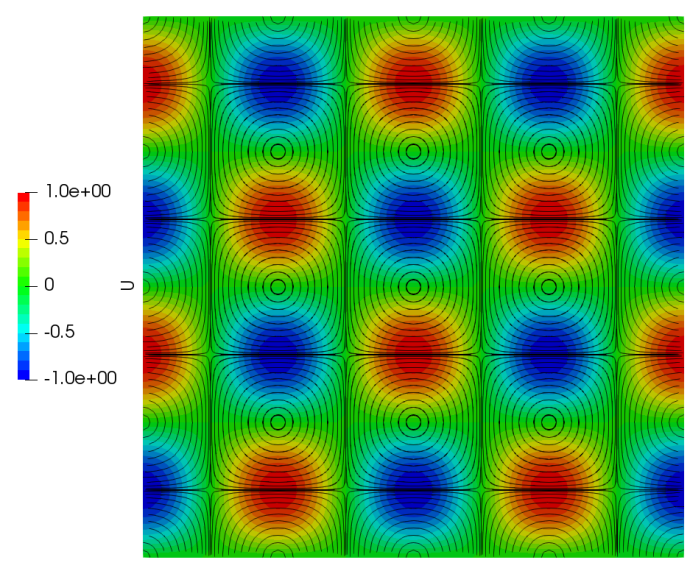

(a) $u$-velocity

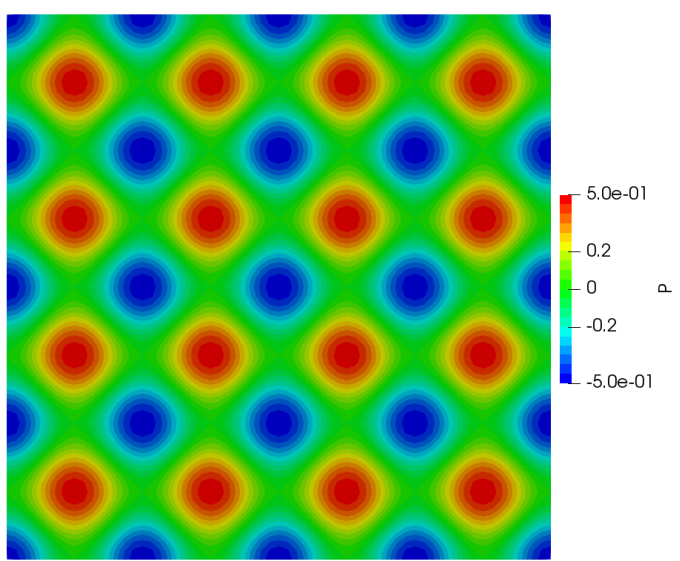

(b) pressure

Figure 10: Initial velocity and pressure field for the Taylor-Green vortex problem. The streamlines are shown on top of the velocity field as a reference.

analytic solution for the kinetic energy that can be constructed from Eq.(86). The curves were normalised with the theoretical kinetic energy at the beginning of the simulation. It can be seen that all schemes for both methods and the tested Reynolds numbers, with the exception of the AC method at $R e=100$, dissipate the kinetic energy faster than the expected solution. This is in line with observations made by Oger et al. [63] and Khayyer et al. [64], who both showed the decay of kinetic energy at $R e=100$ and $R e=1000$, respectively, using the Smoothed Particle Hydrodynamics (SPH) method. Oger et al. performed a sensitivity study and showed that an increase of particles along the $x$ - and $y$-direction was sufficient to improve the kinetic energy decay rate. However, they also showed that sufficiently accurate results were still obtained using their coarsest initial particle distribution of 50 particles in each direction. Since we are interested in the relative performance of each scheme, the coarser mesh allows differences among schemes to be seen more clearly while still providing physical correct results. Using similar mesh distributions as in Oger et al., these findings were also confirmed by Khayyer et al. Since both of these aforementioned studies used the SPH method, their computational considerations are slightly different from the Finite Volume method employed in the current study. Referring back to the AC method at $\mathrm{Re}=100$, it can be seen that the SCB, Rusanov and Rusanov+MCB scheme all provide the expected dissipation rates, albeit that all of them show a 


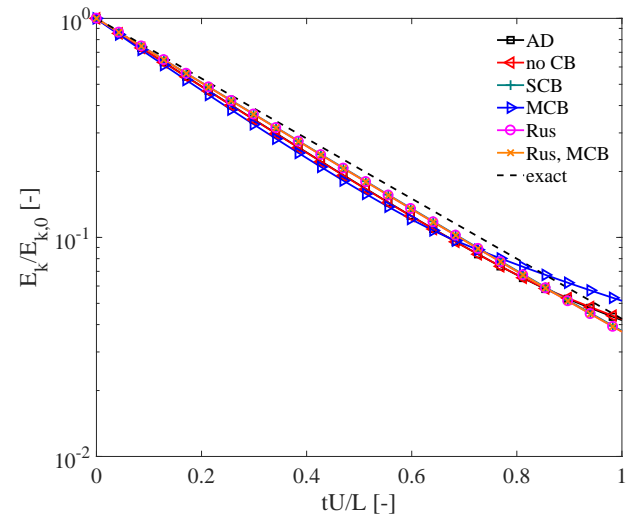

(a) $\mathrm{AC}, \mathrm{Re}=100$

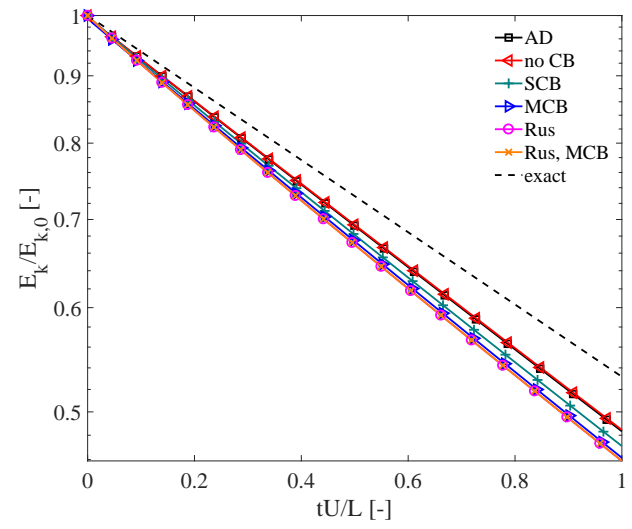

(c) $\mathrm{AC}, \mathrm{Re}=500$

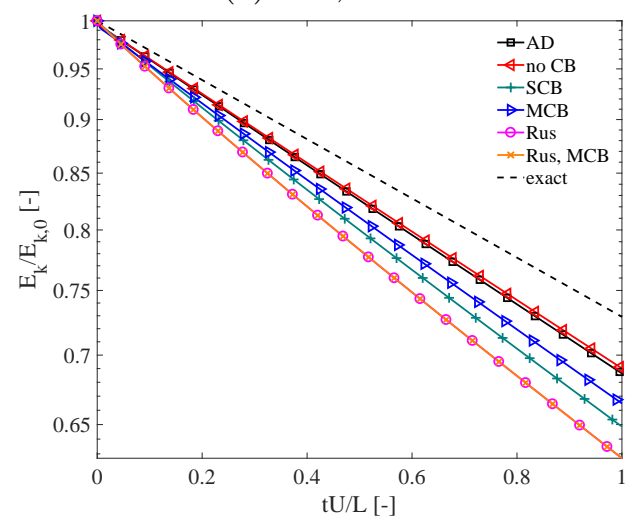

(e) $\mathrm{AC}, \mathrm{Re}=1000$

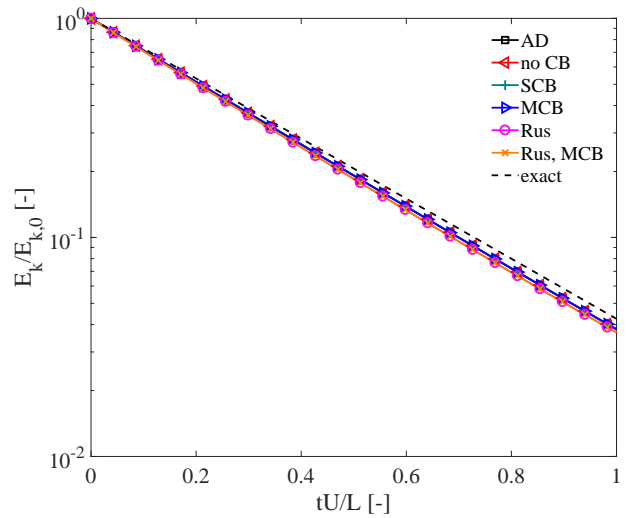

(b) FSAC-PP, Re $=100$

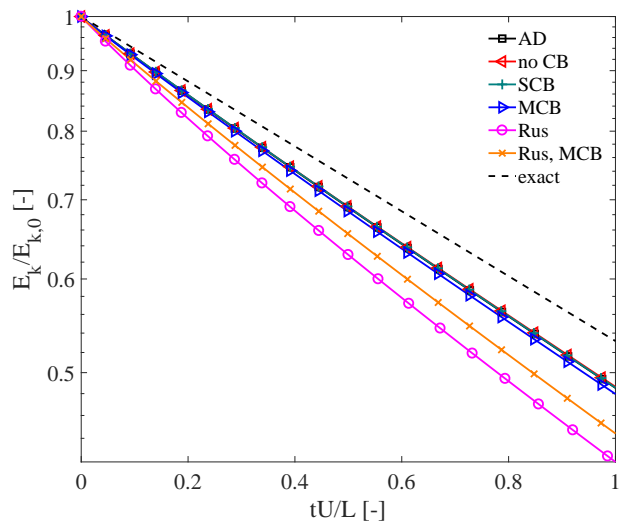

(d) FSAC-PP, Re $=500$

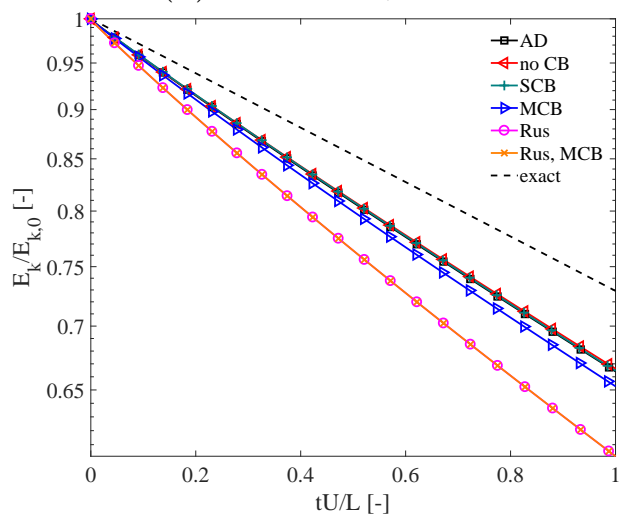

(f) FSAC-PP, Re $=1000$

Figure 11: Decay of kinetic energy for both the AC and FSAC-PP method using different Reynolds numbers for the Taylor-Green vortex problem. 
higher net rate of dissipated kinetic energy. This suggests that the right amount of dissipation is present in the simulation. This is not surprising, as it was established that the Rusanov Riemann solver possess an inherent amount of numerical dissipation. Therefore, its combination with the MCB scheme does possess a similar amount of numerical dissipation. The reason that the SCB scheme performs similarly is rooted in its inherent Godunov Riemann solver, Eq.(79), which is essentially an upwinding procedure, based on the local eigenvalues. As was stated by Ekaterinaris [65], "Upwind-biased schemes, however, based only on formal accuracy (truncation error) inherently introduce some form of artificial smoothing". Thus, even the third order accurate polynomial scheme employed in this study has to sacrifice some of its accuracy in favour of stability through the Riemann solver of Godunov. As was seen in the previous test case, the SCB scheme did indeed promote stability over the non-characteristic treatment which was based on the same polynomial reconstruction scheme. Both the non-characteristic and MCB treatment, as well as the central scheme with artificial dissipation show that they struggle to produce the correct dissipation rate once the vortices start to decay. The FSAC-PP method, on the other hand, shows very little difference among the schemes at $R e=100$. This can be attributed again to the smoothing properties of the elliptic pressure Poisson solver that has also provided scheme independent-like solutions at low Reynolds number for the lid driven cavity problem and in the literature [37, 38]. Morinishi et al. [66] investigated different numerical schemes on regular, staggered and collocated mesh arrangements and investigated their conservative properties. They showed that simultaneously conservation of momentum and kinetic energy is only achievable with specialised numerical schemes. Their schemes were losing the conservative properties if the order of accuracy was increased beyond second-order on non-uniform meshes. Despite the use of a regular mesh in this study, there are indicators which suggests that the departure from the kinetic energy decay rate is indeed rooted in the reconstruction scheme used in this study. First of all, we solve the momentum equation in non-conservative form according to Eq.(2) and Eq.(4) with respect to the non-linear term. According to Morinishi et al. [66], a fully conservative momentum equation is a prerequisite to construct fully conservative interpolation schemes. Secondly, we purposefully have chosen a low-dissipative numerical scheme to keep the inherent artificial dissipation low with the aim to provide locally scaled numerical dissipation through the Riemann solver. We can see in-fact that the MCB scheme relying solely on the third-order reconstruction scheme is not 
conserving energy while its combination with the Rusanov Riemann solver is at $\mathrm{Re}=100$ using the $\mathrm{AC}$ method. It was furthermore stated in [66] that a collocated primitive variable arrangement for unsteady flows may result in larger errors. For increasing Reynolds numbers, all schemes produce a decay rate lower than the expected rate, which is again in agreement with Khayyer et al. [64] at Re=1000. It should be noted here that the initial bumps observed by Khayyer et al. are due to the SPH method and thus absent in the current plots. However, as discussed above, the Finite volume approach can have its own characteristics in the energy decay.

At $R e=500$, it can be seen that the Rusanov Riemann solver in conjunction with the MCB scheme shows improved decay rates over the Rusanov Riemann solver alone, when using the FSAC-PP method. For the AC method there is little difference between these two schemes. At Re=1000, however, both schemes produce very similar results for both methods. Furthermore, it can also be confirmed that the Rusanov Riemann solver is the most dissipative approach in the current case, especially at high Reynolds numbers. It is positive to note here, however, that the added numerical dissipation within the Rusanov Riemann solver does not adversely affect the overall solution at low Reynolds numbers. Despite the discrepancies at $\mathrm{Re}=100$, it is the non-characteristic and central scheme with artificial dissipation, that produces the best agreement with the expected dissipation rate for Reynolds number of $\mathrm{Re}=500$ and above for both methods. It can also be seen that for an increase in Reynolds number, the MCB scheme seems to perform better than the SCB scheme using the AC method. While the MCB scheme showed a departure from the expected decay rate at $\mathrm{Re}=100$, it shows comparable decay rates at $\mathrm{Re}=500$ compared to the SCB scheme and even improves at $\mathrm{Re}=1000$. This is another indicator that the anisotropic effects, that may be introduced as the Reynolds number increases, may be better captured by a multi-directional approached compared to the single-directional one. Using the FSAC-PP method, however, the MCB and SCB scheme show very similar performance even at $\mathrm{Re}=1000$.

Finally, we would like to point out that a more favourable decay in kinetic energy may be obtained using a less dissipative Riemann solver, for example the HLL or HLLC Riemann solver, which both respect the non-linearity of the Navier-Stokes equations. It should be noted, however, that an extension is not straight-forward for the FSAC-PP method for which the pressure bias needs to be removed first, as was shown by Smith et al. [45]. 


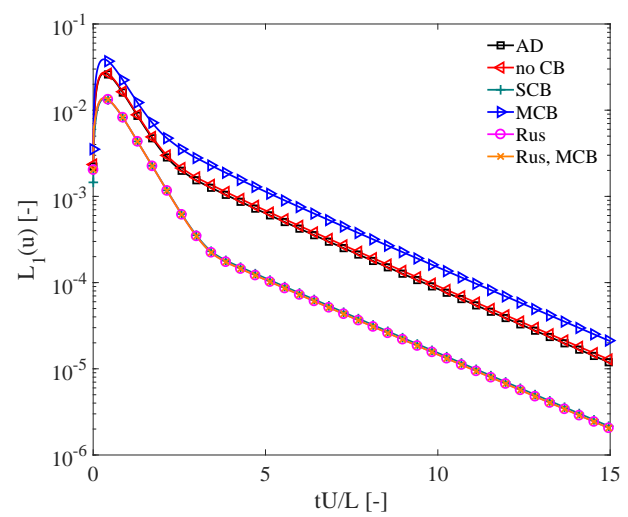

(a) $\mathrm{AC}, \mathrm{Re}=100$

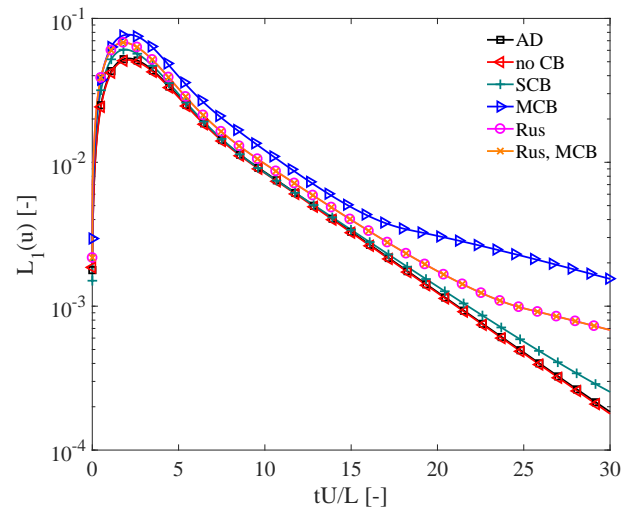

(c) $\mathrm{AC}, \mathrm{Re}=500$

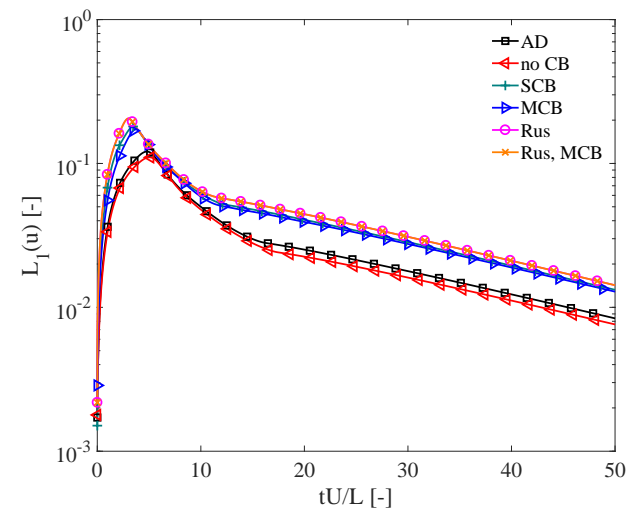

(e) $\mathrm{AC}, \mathrm{Re}=1000$

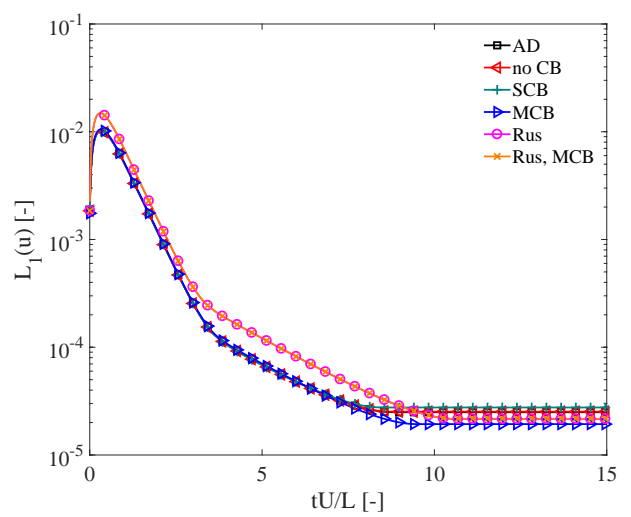

(b) FSAC-PP, Re $=100$

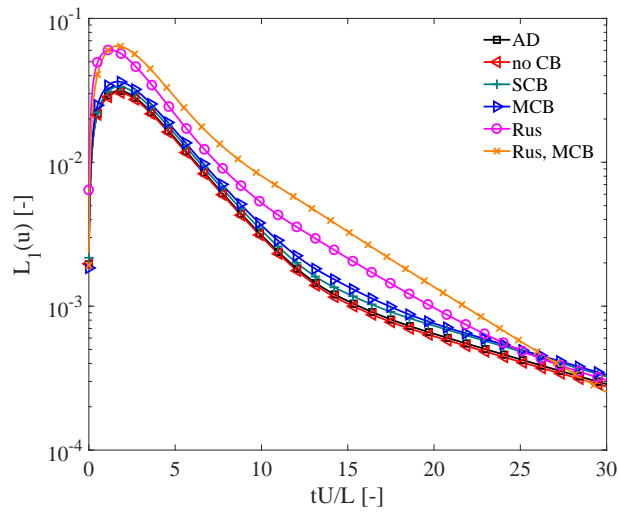

(d) FSAC-PP, Re $=500$

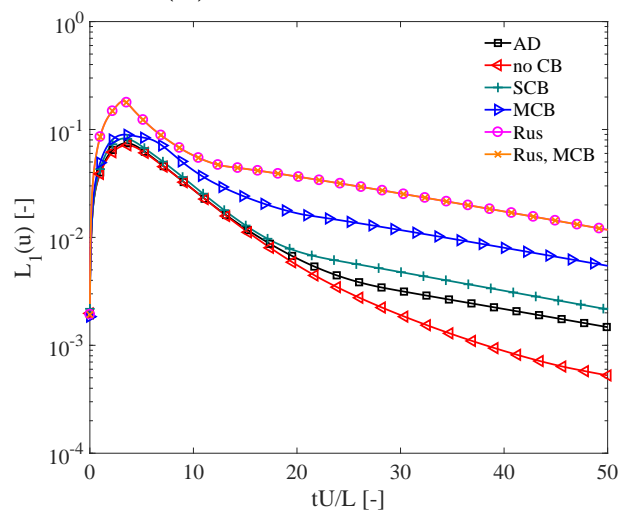

(f) FSAC-PP, Re $=1000$

Figure 12: Evolution of the $L_{1}$ error norm of the u-velocity component for both the $\mathrm{AC}$ and FSAC-PP method using different Reynolds numbers for the Taylor-Green vortex problem. 
To further elaborate on the accuracy of each scheme and its role on the velocity field, we plot the $L_{1}$ error norm of the $u$-velocity component which was averaged over the entire domain for each time step and is shown in Figure 12. It is a useful measure as the decay in kinetic energy only provides information on the relative error compared to the exact solution, while the error norm provides an absolute comparison to the exact solution.

For all error curves, we see an initial increase in error which then decays over time with varying slopes. The peak of the error increases for both methods as the Reynolds number is increased, indicating that the numerical viscosity becomes dominant with decreasing physical viscosity. At the lowest Reynolds number of $\mathrm{Re}=100$, it can be seen that the $\mathrm{AC}$ method experiences a linear decrease in log space for the error while the FSAC-PP method shows three distinct regions with different slopes. After the initial increase in error, the FSAC-PP method produces steeper error reduction curves. These slopes are then reduced before plateauing for all schemes. It follows from the initial steeper slopes that the overall error is lower using the FSAC-PP method over the tested period which is also confirmed by Table 5 and 6 . The same behaviour was observed by Könözsy and Drikakis [38], where the FSAC-PP method was tested for various examples at $R e=100$. They showed that the FSAC-PP method has a tendency to reduce the error faster than the AC method and then starts to flatten to a point at which the error does not change anymore. This is an inherent property of the FSAC-PP method and the same behaviour could be observed in the present test case. For higher Reynolds numbers, the errors decay monotonically for both methods after the initial error peak. At $\mathrm{Re}=500$, we can observe that the MCB scheme produces the highest error with the $\mathrm{AC}$ method while it produces errors close to the best error curves with the FSAC-PP method. Furthermore, the pairing of the Rusanov Riemann solver and the MCB scheme produces initially the highest error while its steeper slope is able to reduce the error the most so that at the end of the simulation its error has become the lowest of all schemes. Its peak in error is initially comparable to that of the Rusanov Riemann solver alone which is to be expected, as the peak in error is solely due to the numerical dissipation in the Rusanov Riemann solver. The MCB scheme, low in dissipation by design, is not increasing this error, rather it provides a mechanism to reduce the error fastest, albeit at an initially higher overall error. For the highest tested Reynolds number of $R e=1000$, both the Rusanov Riemann solver and its hybridisation with the MCB scheme produce similar results using both methods. For both the $\mathrm{Re}=500$ and $\mathrm{Re}=1000$ case, 
it is again the non-characteristic scheme which produces the most accurate results. This may be seen as a disadvantage of the Rusanov approach and the MCB scheme by either itself or in combination with the Riemann solver, however, the reader is reminded that the Rusanov Riemann solver has been primarily used to extend the MCB scheme to higher Reynolds numbers. In the present case the Navier-Stokes equations may not be sufficiently challenged at the moderate Reynolds number of $R e=1000$ so that the scheme with the lowest inherent numerical dissipation produces the best agreement. This is in-fact the case here where the non-CB scheme relies on the lowdissipative polynomial reconstruction. Furthermore, the current test case is initialised with an already developed vortical flow field. These vortical structures are merely decayed over time and so do not challenge the solver in form of predicting vortical flow structures, rather, the current test case investigates how well vortical flow structure are preserved. Thus, it would be wrong to dismiss the more dissipative results of the Rusanov Riemann solver in favour of the non-characteristic approach. We saw for the lid driven cavity case that at higher Reynolds numbers, where the vortical flow field needs to be established by the solver and thus presents a greater challenge, the $\mathrm{Ru}$ sanov Riemann solver provided stability for all tested Reynolds numbers and grid sizes where the non-characteristic approach struggled due to the loss of physical and numerical dissipation. We can see from the current results on the Taylor-Green vortex problem, however, that the additional numerical dissipation inherent to the Rusanov Riemann solver and Godunov's Riemann solver in the SCB scheme do have an effect on the accuracy while providing reasonable errors curves.

Figure 13 shows the evolution of the $L_{1}$ error norm in pressure for both AC and FSAC-PP method across the different tested Reynolds numbers. This is of particular interest as the FSAC-PP method is very similar to the AC method, the difference being the removed pressure gradient from the momentum equation which then is solved in a second Fractional-Step through the pressure Poisson equation from the Pressure Projection method of Chorin. Thus, the pressure presents an important quantity to establish comparisons between the AC and FSAC-PP method. As can be seen from Figure 13, the pressure is decreasing over time. Unlike the $L_{1}$ norm of the velocity component, there is no clear peak structure in the error of the pressure. The AC method does observe a small increase in error at $\mathrm{Re}=500$ and $\mathrm{Re}=1000$ and so does the FSAC-PP method at $\mathrm{Re}=1000$, however, below these values a 


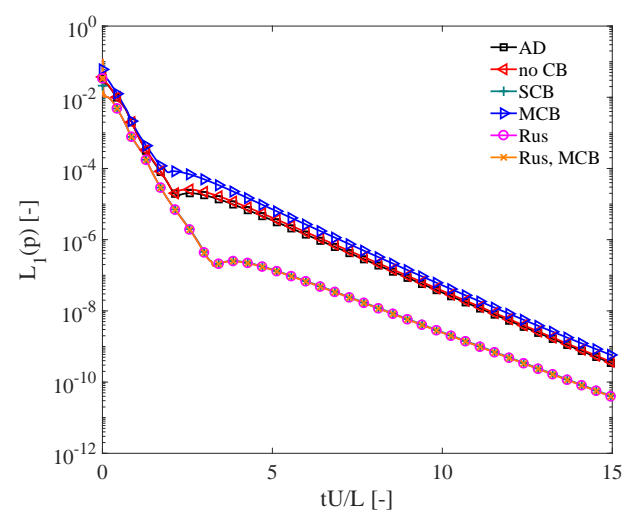

(a) $\mathrm{AC}, \mathrm{Re}=100$

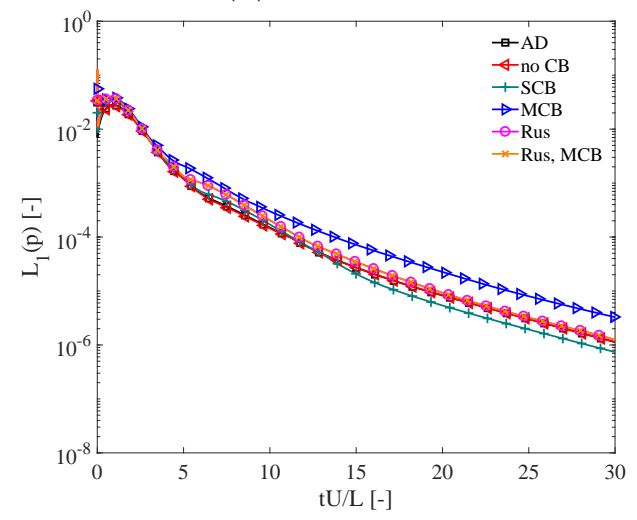

(c) $\mathrm{AC}, \mathrm{Re}=500$

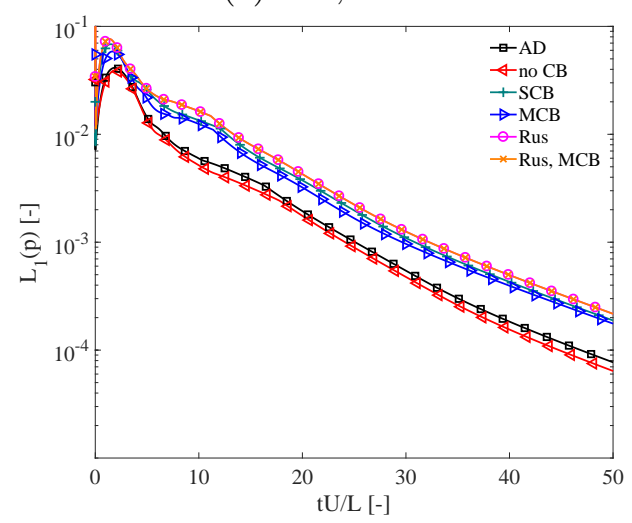

(e) $\mathrm{AC}, \mathrm{Re}=1000$

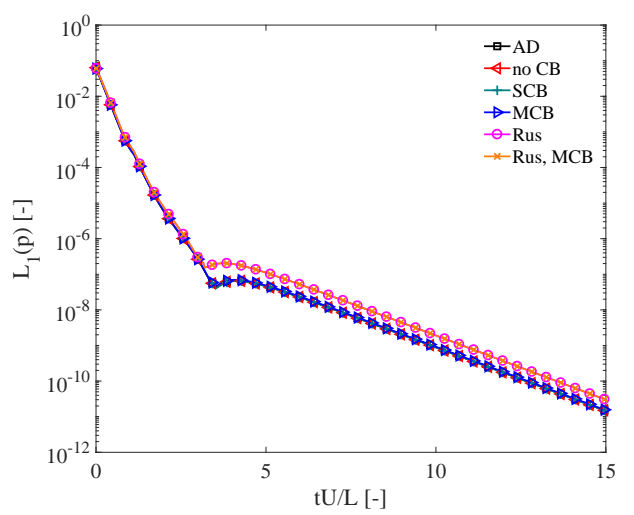

(b) FSAC-PP, Re $=100$

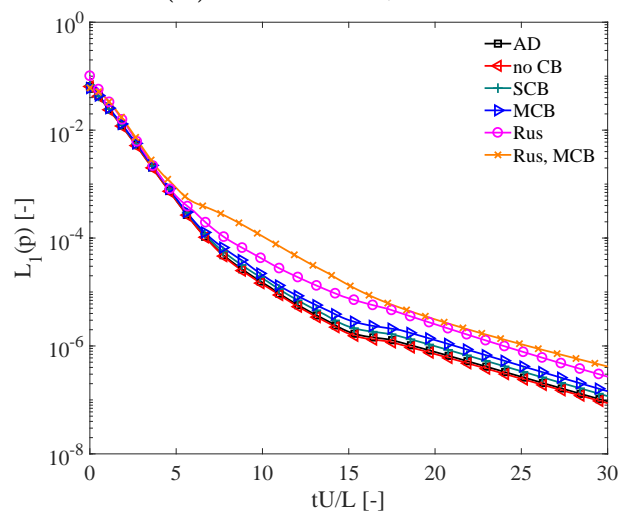

(d) FSAC-PP, Re $=500$

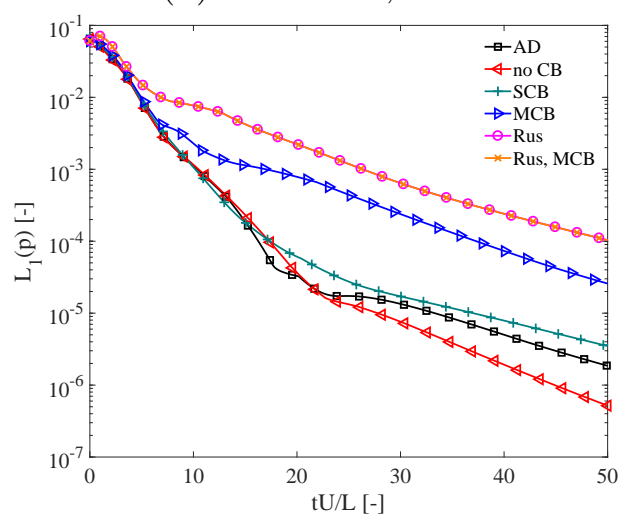

(f) FSAC-PP, Re $=1000$

Figure 13: Evolution of the $L_{1}$ error norm of the pressure for both the AC and FSAC-PP method using different Reynolds numbers for the Taylor-Green vortex problem. 
monotonic decrease in error is achieved.

We can observe similar behaviours of the different numerical schemes as seen for the $L_{1}$ error of the velocity component $u$. The Rusanov Riemann solver and its combination with the MCB scheme provides initially a close agreement at low Reynolds number while its accuracy decreases at higher Reynolds numbers using the FSAC-PP method. The non-CB scheme provides the best agreement for both methods at $\mathrm{Re}=1000$ which is again due to its less inherent numerical dissipation. However, another aspect emerges from this discussion which provides further insight in the comparison among the AC and FSAC-PP method themselves. At Re=100, both methods provide very similar error curves. The FSAC-PP method may have closer agreement between the different schemes, but overall all schemes reduce the error in pressure to approximately $10^{-10}$ for both methods. At $\operatorname{Re}=500$, however, the discrepancies start to show more prominent. Here, the non-characteristic approach, as well as the central scheme with artificial dissipation both produce errors of approximately $10^{-6}$ using the $\mathrm{AC}$ method while this value becomes $10^{-7}$ using the FSAC-PP method. At $\mathrm{Re}=1000$, the same schemes just drop below $10^{-4}$ for the AC method and $10^{-6}$ for the FSAC-PP method. This order of magnitude difference in the error for the pressure is again attributed to the more physical treatment of the pressure in the FSAC-PP method, where the momentum equation in the first Fractional-Step is fully hyperbolic which is then coupled with the elliptic Poisson solver in the second Fractional-Step for the pressure. This elliptic and implicit treatment of the pressure promotes stability and a smoothed pressure field. Furthermore, while some incompressible methods require the linearisation of the convective term in order to provide stability at the expense of losing the non-linear behaviour of the Navier-Stokes equations, we are able to smooth and stabilise the pressure through the elliptic and implicit Poisson equation. This is already a linear equation and does not require linearisation and so we are able to numerically solve the fully non-linear Navier-Stokes equations. Since the pressure is smoothed, which is driving the flow, instabilities are damped and a non-linear solution with rather low error values for the pressure is obtained. This shows that the numerical method used, especially its numerical character, has a large influence on the overall performance of the method.

Table 5 and 6 summarise the previous discussion quantitatively. Here, the $L_{0}$ and $L_{1}$ error norms for velocity and pressure are shown for each Reynolds number, which were obtained by integrating the errors over time. It can be 
Table 5: Integrated $L_{0}$ and $L_{1}$ error norms for both velocity components and the pressure over time. Results are shown for the AC method.

\begin{tabular}{|c|c|c|c|c|c|c|c|}
\hline \multirow[b]{2}{*}{$\mathrm{Re}$} & & \multirow{2}{*}{$\frac{\mathrm{AD}}{\mathrm{no} \mathrm{CB}}$} & \multicolumn{3}{|c|}{ no Riemann Solver } & \multicolumn{2}{|c|}{ Rusanov Riemann Solver } \\
\hline & & & no $\mathrm{CB}$ & $\mathrm{SCB}$ & $\mathrm{MCB}$ & no $\mathrm{CB}$ & $\mathrm{MCB}$ \\
\hline \multirow{6}{*}{100} & $L_{0}(u)[\%]$ & $3.47 \mathrm{e}-05$ & $3.56 \mathrm{e}-05$ & $1.80 \mathrm{e}-05$ & $4.97 \mathrm{e}-05$ & $1.85 \mathrm{e}-05$ & $1.85 \mathrm{e}-05$ \\
\hline & $L_{0}(v)[\%]$ & $3.47 \mathrm{e}-05$ & $3.56 \mathrm{e}-05$ & $1.80 \mathrm{e}-05$ & $4.97 \mathrm{e}-05$ & $1.85 \mathrm{e}-05$ & $1.85 \mathrm{e}-05$ \\
\hline & $L_{0}(p)[\%]$ & $1.44 \mathrm{e}-05$ & $1.46 \mathrm{e}-05$ & $8.52 \mathrm{e}-06$ & $1.77 \mathrm{e}-05$ & 8.80e-06 & 8.80e-06 \\
\hline & $L_{1}(u)[\%]$ & 7.83e-06 & $8.09 \mathrm{e}-06$ & $3.61 \mathrm{e}-06$ & $1.17 \mathrm{e}-05$ & $3.68 \mathrm{e}-06$ & $3.68 \mathrm{e}-06$ \\
\hline & $L_{1}(v)[\%]$ & $7.83 \mathrm{e}-06$ & $8.09 \mathrm{e}-06$ & $3.61 \mathrm{e}-06$ & $1.17 \mathrm{e}-05$ & $3.68 \mathrm{e}-06$ & $3.68 \mathrm{e}-06$ \\
\hline & $L_{1}(p)[\%]$ & $2.55 \mathrm{e}-06$ & $2.68 \mathrm{e}-06$ & $1.27 \mathrm{e}-06$ & $3.53 \mathrm{e}-06$ & $1.43 \mathrm{e}-06$ & $1.43 \mathrm{e}-06$ \\
\hline \multirow{6}{*}{500} & $L_{0}(u)[\%]$ & $2.94 \mathrm{e}-04$ & $2.89 \mathrm{e}-04$ & $3.24 \mathrm{e}-04$ & $4.23 \mathrm{e}-04$ & $3.64 \mathrm{e}-04$ & $3.64 \mathrm{e}-04$ \\
\hline & $L_{0}(v)[\%]$ & $2.94 \mathrm{e}-04$ & $2.89 \mathrm{e}-04$ & $3.24 \mathrm{e}-04$ & $4.23 \mathrm{e}-04$ & $3.64 \mathrm{e}-04$ & $3.64 \mathrm{e}-04$ \\
\hline & $L_{0}(p)[\%]$ & $7.62 \mathrm{e}-05$ & $7.41 \mathrm{e}-05$ & $8.72 \mathrm{e}-05$ & $9.61 \mathrm{e}-05$ & $9.34 \mathrm{e}-05$ & $9.34 \mathrm{e}-05$ \\
\hline & $L_{1}(u)[\%]$ & $7.64 \mathrm{e}-05$ & $7.49 \mathrm{e}-05$ & $8.33 \mathrm{e}-05$ & $1.14 \mathrm{e}-04$ & $9.46 \mathrm{e}-05$ & $9.46 \mathrm{e}-05$ \\
\hline & $L_{1}(v)[\%]$ & $7.64 \mathrm{e}-05$ & $7.49 \mathrm{e}-05$ & $8.33 \mathrm{e}-05$ & $1.14 \mathrm{e}-04$ & $9.46 \mathrm{e}-05$ & $9.46 \mathrm{e}-05$ \\
\hline & $L_{1}(p)[\%]$ & $1.52 \mathrm{e}-05$ & $1.50 \mathrm{e}-05$ & $1.76 \mathrm{e}-05$ & $2.08 \mathrm{e}-05$ & $1.96 \mathrm{e}-05$ & $1.96 \mathrm{e}-05$ \\
\hline \multirow{6}{*}{1000} & $L_{0}(u)[\%]$ & $1.13 \mathrm{e}-03$ & $1.06 \mathrm{e}-03$ & $1.56 \mathrm{e}-03$ & $1.45 \mathrm{e}-03$ & $1.67 \mathrm{e}-03$ & $1.67 \mathrm{e}-03$ \\
\hline & $L_{0}(v)[\%]$ & $1.13 \mathrm{e}-03$ & $1.06 \mathrm{e}-03$ & $1.56 \mathrm{e}-03$ & $1.45 \mathrm{e}-03$ & $1.67 \mathrm{e}-03$ & $1.67 \mathrm{e}-03$ \\
\hline & $L_{0}(p)[\%]$ & $2.04 \mathrm{e}-04$ & $1.91 \mathrm{e}-04$ & $3.04 \mathrm{e}-04$ & $2.75 \mathrm{e}-04$ & $3.30 \mathrm{e}-04$ & $3.30 \mathrm{e}-04$ \\
\hline & $L_{1}(u)[\%]$ & $3.68 \mathrm{e}-04$ & $3.41 \mathrm{e}-04$ & $5.40 \mathrm{e}-04$ & $5.15 \mathrm{e}-04$ & $5.87 \mathrm{e}-04$ & $5.87 \mathrm{e}-04$ \\
\hline & $L_{1}(v)[\%]$ & $3.68 \mathrm{e}-04$ & $3.41 \mathrm{e}-04$ & $5.40 \mathrm{e}-04$ & $5.15 \mathrm{e}-04$ & $5.87 \mathrm{e}-04$ & $5.87 \mathrm{e}-04$ \\
\hline & $L_{1}(p)[\%]$ & $5.75 \mathrm{e}-05$ & $5.31 \mathrm{e}-05$ & $9.84 \mathrm{e}-05$ & $8.68 \mathrm{e}-05$ & $1.11 \mathrm{e}-04$ & $1.11 \mathrm{e}-04$ \\
\hline
\end{tabular}

seen that the integrated $L_{1}$ error norm of the velocity components are generally lower with the FSAC-PP method compared to the AC method across the tested schemes and Reynolds number, with a few exceptions. Notably, the MCB scheme at $\mathrm{Re}=100$ produces error norms which are an order of magnitude lower when using the FSAC-PP method. This difference decreases at higher Reynolds numbers while the FSAC-PP method still produces better results. Similar observations can be made about the Rusanov Riemann solver and its combination with the MCB scheme, where lower integrated 
Table 6: Integrated $L_{0}$ and $L_{1}$ error norms for both velocity components and the pressure over time. Results are shown for the FSAC-PP method.

\begin{tabular}{|c|c|c|c|c|c|c|c|}
\hline \multirow[b]{2}{*}{$\mathrm{Re}$} & & \multirow{2}{*}{$\frac{\mathrm{AD}}{\mathrm{no} \mathrm{CB}}$} & \multicolumn{3}{|c|}{ no Riemann Solver } & \multicolumn{2}{|c|}{ Rusanov Riemann Solver } \\
\hline & & & no $\mathrm{CB}$ & SCB & $\mathrm{MCB}$ & no $\mathrm{CB}$ & $\mathrm{MCB}$ \\
\hline \multirow{6}{*}{100} & $L_{0}(u)[\%]$ & $1.41 \mathrm{e}-05$ & $1.41 \mathrm{e}-05$ & $1.42 \mathrm{e}-05$ & $1.44 \mathrm{e}-05$ & $1.93 \mathrm{e}-05$ & $1.93 \mathrm{e}-05$ \\
\hline & $L_{0}(v)[\%]$ & $1.41 \mathrm{e}-05$ & $1.41 \mathrm{e}-05$ & $1.42 \mathrm{e}-05$ & $1.44 \mathrm{e}-05$ & $1.93 \mathrm{e}-05$ & $1.93 \mathrm{e}-05$ \\
\hline & $L_{0}(p)[\%]$ & $1.02 \mathrm{e}-05$ & $1.01 \mathrm{e}-05$ & $1.00 \mathrm{e}-05$ & $1.01 \mathrm{e}-05$ & $1.14 \mathrm{e}-05$ & $1.14 \mathrm{e}-05$ \\
\hline & $L_{1}(u)[\%]$ & $2.78 \mathrm{e}-06$ & $2.77 \mathrm{e}-06$ & $2.78 \mathrm{e}-06$ & $2.83 \mathrm{e}-06$ & $3.86 \mathrm{e}-06$ & $3.86 \mathrm{e}-06$ \\
\hline & $L_{1}(v)[\%]$ & $2.78 \mathrm{e}-06$ & $2.77 \mathrm{e}-06$ & $2.78 \mathrm{e}-06$ & $2.83 \mathrm{e}-06$ & $3.86 \mathrm{e}-06$ & $3.86 \mathrm{e}-06$ \\
\hline & $L_{1}(p)[\%]$ & $2.71 \mathrm{e}-06$ & $2.67 \mathrm{e}-06$ & $2.65 \mathrm{e}-06$ & $2.66 \mathrm{e}-06$ & $2.84 \mathrm{e}-06$ & $2.84 \mathrm{e}-06$ \\
\hline \multirow{6}{*}{500} & $L_{0}(u)[\%]$ & $1.70 \mathrm{e}-04$ & $1.66 \mathrm{e}-04$ & $1.80 \mathrm{e}-04$ & $1.93 \mathrm{e}-04$ & $2.88 \mathrm{e}-04$ & $3.31 \mathrm{e}-04$ \\
\hline & $L_{0}(v)[\%]$ & $1.70 \mathrm{e}-04$ & $1.66 \mathrm{e}-04$ & $1.80 \mathrm{e}-04$ & $1.93 \mathrm{e}-04$ & $2.88 \mathrm{e}-04$ & $3.31 \mathrm{e}-04$ \\
\hline & $L_{0}(p)[\%]$ & $6.88 \mathrm{e}-05$ & $6.79 \mathrm{e}-05$ & $6.93 \mathrm{e}-05$ & $7.16 \mathrm{e}-05$ & $8.13 \mathrm{e}-05$ & $8.75 \mathrm{e}-05$ \\
\hline & $L_{1}(u)[\%]$ & $4.11 \mathrm{e}-05$ & $4.01 \mathrm{e}-05$ & $4.40 \mathrm{e}-05$ & $4.73 \mathrm{e}-05$ & $7.07 \mathrm{e}-05$ & $8.44 \mathrm{e}-05$ \\
\hline & $L_{1}(v)[\%]$ & $4.11 \mathrm{e}-05$ & $4.00 \mathrm{e}-05$ & $4.40 \mathrm{e}-05$ & $4.73 \mathrm{e}-05$ & $7.06 \mathrm{e}-05$ & $8.44 \mathrm{e}-05$ \\
\hline & $L_{1}(p)[\%]$ & $1.70 \mathrm{e}-05$ & $1.67 \mathrm{e}-05$ & $1.70 \mathrm{e}-05$ & $1.75 \mathrm{e}-05$ & $2.20 \mathrm{e}-05$ & $2.15 \mathrm{e}-05$ \\
\hline \multirow{6}{*}{1000} & $L_{0}(u)[\%]$ & $6.58 \mathrm{e}-04$ & $6.46 \mathrm{e}-04$ & $7.68 \mathrm{e}-04$ & $1.02 \mathrm{e}-03$ & $1.54 \mathrm{e}-03$ & $1.53 \mathrm{e}-03$ \\
\hline & $L_{0}(v)[\%]$ & $6.59 \mathrm{e}-04$ & $6.46 \mathrm{e}-04$ & $7.67 \mathrm{e}-04$ & $1.02 \mathrm{e}-03$ & $1.54 \mathrm{e}-03$ & $1.54 \mathrm{e}-03$ \\
\hline & $L_{0}(p)[\%]$ & $1.70 \mathrm{e}-04$ & $1.68 \mathrm{e}-04$ & $1.75 \mathrm{e}-04$ & $1.86 \mathrm{e}-04$ & $2.49 \mathrm{e}-04$ & $2.49 \mathrm{e}-04$ \\
\hline & $L_{1}(u)[\%]$ & $1.74 \mathrm{e}-04$ & $1.61 \mathrm{e}-04$ & $1.96 \mathrm{e}-04$ & $2.92 \mathrm{e}-04$ & $5.13 \mathrm{e}-04$ & $5.13 \mathrm{e}-04$ \\
\hline & $L_{1}(v)[\%]$ & $1.74 \mathrm{e}-04$ & $1.61 \mathrm{e}-04$ & $1.96 \mathrm{e}-04$ & $2.93 \mathrm{e}-04$ & $5.13 \mathrm{e}-04$ & $5.13 \mathrm{e}-04$ \\
\hline & $L_{1}(p)[\%]$ & $4.35 \mathrm{e}-05$ & $4.27 \mathrm{e}-05$ & $4.47 \mathrm{e}-05$ & $5.16 \mathrm{e}-05$ & $7.98 \mathrm{e}-05$ & $7.98 \mathrm{e}-05$ \\
\hline
\end{tabular}

$L_{1}$ error norms are obtained using the FSAC-PP method, where only at $\mathrm{Re}=100$ slightly better results occur using the AC method which shows integrated error norms that are $4.7 \%$ lower compared to the FSAC-PP method. Similar observations are also true for the $L_{1}$ error norm of the pressure, where generally better results are obtained using the FSAC-PP method. It is noteworthy here that higher errors are obtained using a Rusanov Riemann solver approach at higher Reynolds numbers, which suggests that its dissipative nature has measurable impact on the solution. On the other hand, the non-CB 
Table 7: Average number of iterations required to converge in pseudo time to the convergence threshold of $\varepsilon=10^{-4}$. Results are shown for the AC and FSAC-PP method and the speed-up of the FSAC-PP method over the AC method is given in parenthesis.

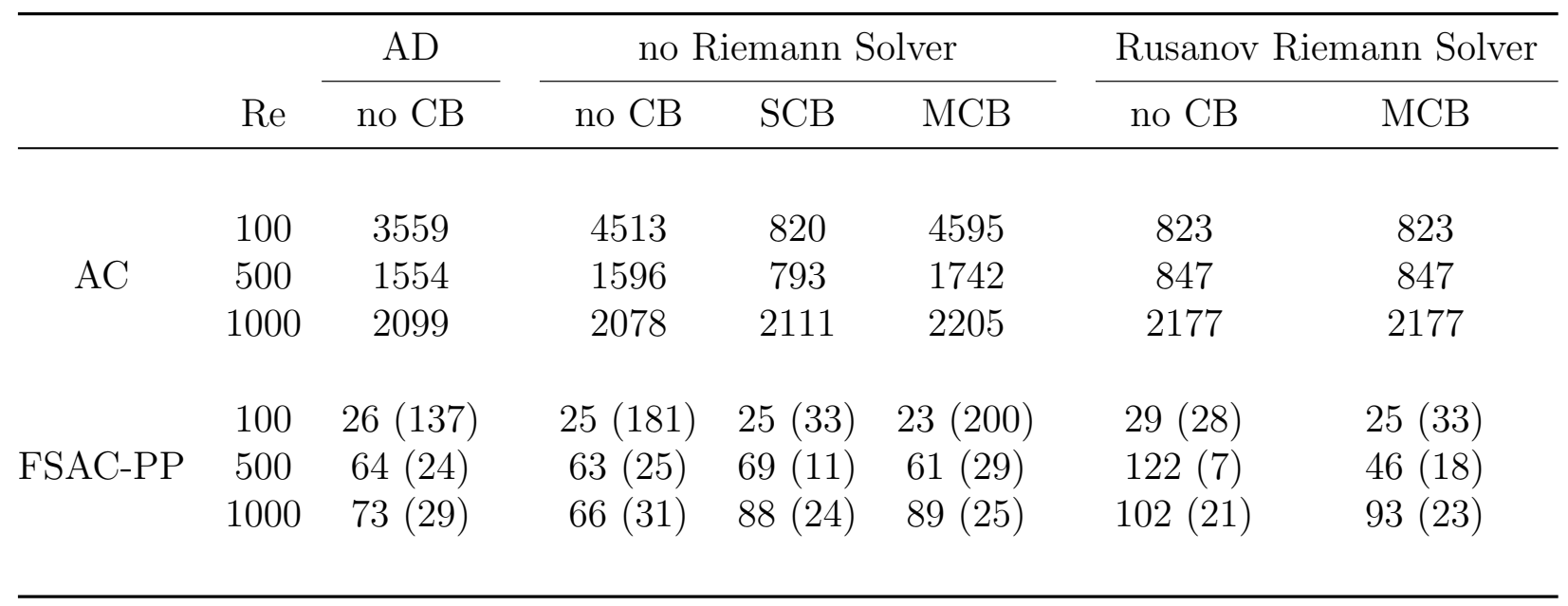

schemes shows consistently a low amount of error, which is in line with the previous discussion on the dissipative properties of the scheme. However, the increase in accuracy comes at the cost of loss in stability at higher Reynolds numbers with challenging flow structures, as was observed for the lid driven cavity case.

Table 7 shows the average number of iterations that were required to converge the solution in pseudo time for each scheme and method at all Reynolds numbers. The speed-up that was gained by the FSAC-PP method over the AC method is further given in parenthesis. It is immediately clear that the FSAC-PP method has significant performance advantages over the $\mathrm{AC}$ method, where the worst speed-up is a factor of 7 and the best speed-up is a factor of 200. With other words, the FSAC-PP method required, at worst, $14.3 \%$ of total simulation time to convergence compared to the AC method or, at best, $0.5 \%$. This makes the FSAC-PP method a prime candidate for unsteady flows, independent of the underlying scheme. Although the MCB scheme requires a substantial amount of iterations using the AC method, especially at $R e=100$, this number can be reduced by pairing the scheme with the Rusanov Riemann solver, which by itself has a lower number of required iterations per pseudo time step. At $\mathrm{Re}=1000$, the number of iterations are reduced and comparable among the different schemes using the AC method. 
For the FSAC-PP method, it can be seen that the Rusanov Riemann solver provides the highest number of required iterations. Using the MCB scheme here, however, provides potential to reduce this number substantially. At $\mathrm{Re}=500$, for example, this number is reduced by a factor of 2.7 , showing the potential of the MCB scheme to increase the convergence rate when using the FSAC-PP method.

In summary, we have seen that not just the scheme, but also the incompressible method that is being used has a significant influence on the accuracy and computational time. Differences up to an order of magnitude in the integrated $L_{1}$ error norm were observed and computational speed ups of a factor of 200 were achieved, which shows the potential of the FSACPP method to treat unsteady flows at a highly improved convergence rate. The Rusanov Riemann solver showed the largest amount of inherent numerical dissipation which was manifested itself in an increased integrated error norm. Although the MCB scheme was not able to reduce this error, it was able to provide significant convergence rate improvements. While the lower dissipative numerical schemes generally showed lower error values, we have seen previously that these schemes have problems to reproduce the correct physical behaviour at higher Reynolds numbers. Thus, a certain amount of dissipation is required and introduced through the Riemann solver which is scaled locally through the eigenvalues of the system.

\subsection{Forced separated flow over a backward-facing step}

We have chosen to present results here for the backward facing step as another classical validation case which deals with forced separation and flow reattachment, the latter being a numerically difficult task to predict correctly. Zamzamian and Razavi [30] also validated their results against the backward facing step problem and based their simulations on the experiments of Denham and Patrick [67], who investigated laminar flows for a step to upstream channel height ratio of $1: 2$. Results were provided for Reynolds numbers of $R e=73,125,191,229$ using an aspect ratio of the inlet channel of $L / h=100 / 15=6 . \dot{6}$. Durst et al. [68] reviewed several studies which measured the downstream location as a function of Reynolds number, at which point a fully developed velocity profile is obtained. They provided an empirical formula of the form

$$
L / h=\left[0.619^{1.6}+(0.0567 \cdot R e)^{1.6}\right]^{1 / 1.6},
$$


Table 8: Grid convergence study for the backward facing step. Shown are the number of elements of the channel in $x\left(n_{x}\right)$ and $y\left(n_{y}\right)$, the normalised reattachment point of the flow behind the step $(x / S)_{\text {re }}$, the extrapolated value using the Richardson extrapolation $\left(\phi^{\text {ext }}\right)$ and the grid convergence index $G C I$.

\begin{tabular}{cccccc}
\hline & $l_{1}$ & $l_{2}$ & $l_{3}$ & $l_{4}$ & $l_{5}$ \\
\hline$n_{x} \times n_{y}$ & $15 \times 38$ & $30 \times 75$ & $60 \times 150$ & $120 \times 300$ & $240 \times 600$ \\
$(x / S)_{\mathrm{re}}$ & 4.60108 & 5.21966 & 5.48584 & 5.60057 & 5.64340 \\
$\phi^{\text {ext }}$ & - & 5.27 & 5.50 & 5.61 & 5.65 \\
$G C I$ & - & 0.6605 & 0.2436 & 0.0994 & 0.0362 \\
\hline
\end{tabular}

for which the aspect ratio can be obtained from the Reynolds number. With this equation and an aspect ratio of $L / h=6 . \dot{6}$, the maximum Reynolds number that can be achieved while still providing a fully developed velocity profile is $R e=116$. This number is, however, only an indication as it has been determined for pure channel flows without expanding channel geometries. None the less, this suggests that the experiments at higher Reynolds numbers do not exhibit a fully developed velocity profile at the intersection of the smaller and larger channel. This can be confirmed by inspecting the velocity profiles provided in [67]. At $R e=125$ and higher, the velocity profiles visually deviate from the fully developed case; even the flow at $R e=73$ shows slight deviations. This creates a major difficulty in terms of the boundary conditions. In order to be consistent with the experiments, we need to sample the velocity profile at the entrance of the larger channel and impose the experimental values in our simulation. We digitized all velocity profiles at the inlet for the different Reynolds numbers and fitted a spline through them. We then interpolated values on the spline which coincided with the locations of the vertices of the mesh and imposed those values in the simulation. Zamzamian and Razavi took the same approach except for the $R e=73$ case, where they imposed a parabolic velocity profile. With the velocity profile given at the entrance to the larger channel, the smaller channel becomes redundant and is removed from the simulation domain. This means, however, that the pressure cannot develop upstream and thus its boundary condition becomes uncertain. In our experience with a full backward facing step geometry, the pressure plays a dominant role in determining the reattachment length and development of the overall flow. For example, 
different pressure boundary conditions applied to the convex corner point at which the forced separation occurs can have measurable difference on the reattachment length. Despite the aforementioned difficulties, we decided to replicate the study of Zamzamian and Razavi using the same reference data and numerical set up of the boundary conditions to compare our results.

We first present a grid convergence study to show at which mesh size a grid independent solution can be obtained. The results are shown in Table 8 using the $\mathrm{AC}$ method at $R e=73$. Here we show the number of elements for each grid level, the length of the reattachment point normalised by the step height, the expected value using the Richardson extrapolation and the grid convergence index (GCI) according to Roache [69]. The reattachment point was determined as the zero-crossing of the skin friction coefficient at the wall. For the current study, we have chosen mesh level $l_{4}$ for all subsequent simulations as the change in the reattachment point to the next finer mesh $\left(l_{5}\right)$ dropped below $1 \%$. At the same time, the computational times were kept at a reasonable level. We use a convergence parameter of $\varepsilon=10^{-8}$ for all simulations.

Figure 14 and 15 show the velocity profiles for the AC and FSAC-PP method using the various combinations of CB schemes and Riemann solver at $R e=229$ at different downstream locations. The non-CB and central scheme with artificial dissipation show almost the same result and most of the velocity profiles overlap. They do, however, show the best match with the reference data for both methods. For the AC method, the SCB, MCB and both Rusanov Riemann solver-based schemes show an increased momentum. For the FSAC-PP method, it is the SCB scheme which produces an excess momentum while the MCB scheme, the Rusanov Riemann solver and the combination of the two show closer agreement with the reference data. As stated by Denham and Patrick, at higher Reynolds numbers there is a tendency for the flow to separate at the upper wall. They did not observe such a behaviour and we could confirm with our simulation that no recirculation at the upper wall is present. However, the flow starts to deflect downwards at the upper wall, effectively reducing the cross-sectional area seen by the flow, which may cause acceleration of the flow at the centreline. The boundary conditions play a crucial role here and it is likely that the inherent uncertainty in those boundary conditions, as discussed above, may have a significant influence on the behaviour of the schemes. 

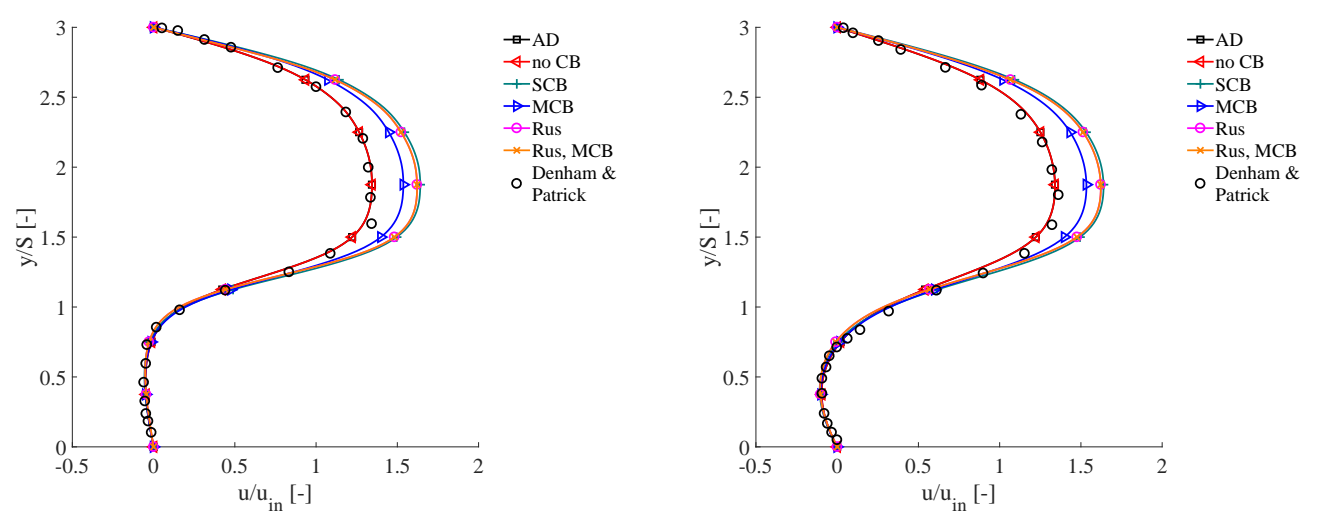

(a) $\mathrm{x} / \mathrm{S}=0.8$
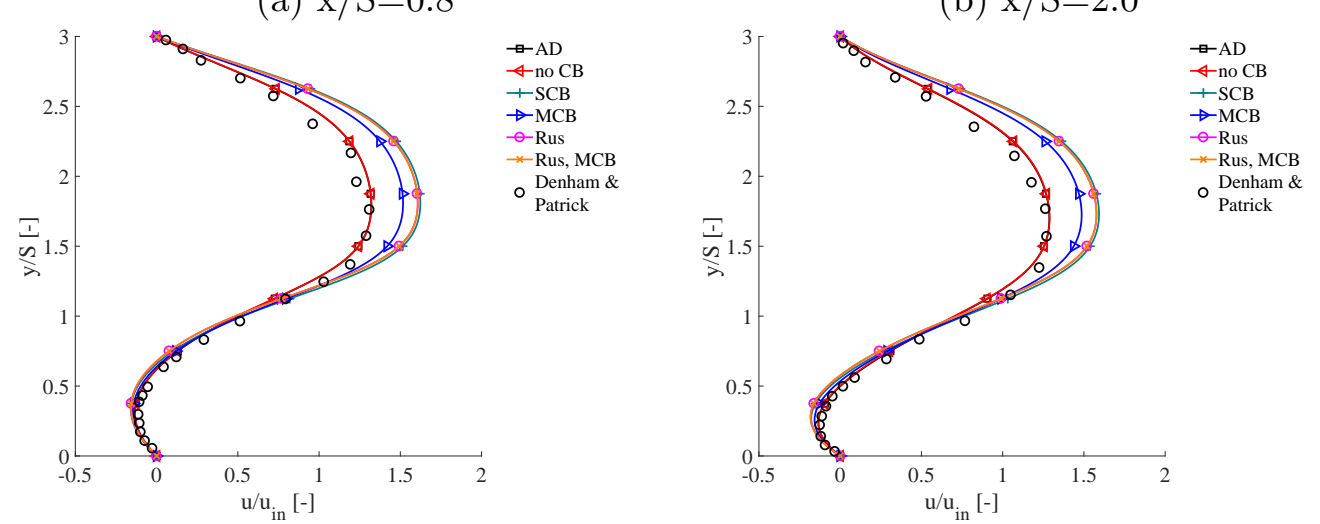

(c) $\mathrm{x} / \mathrm{S}=4.0$

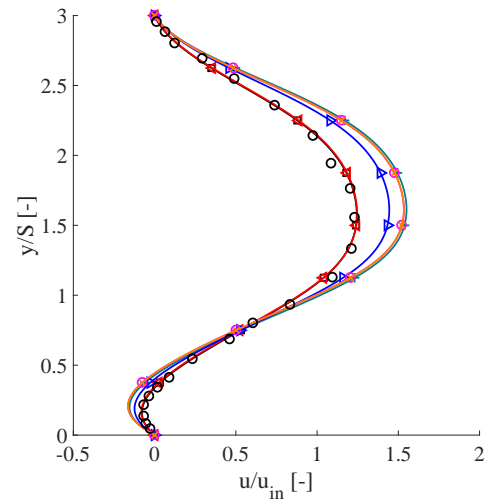

(e) $\mathrm{x} / \mathrm{S}=8.0$

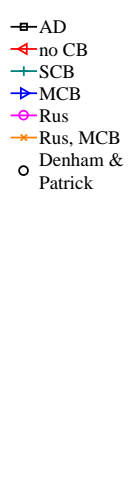

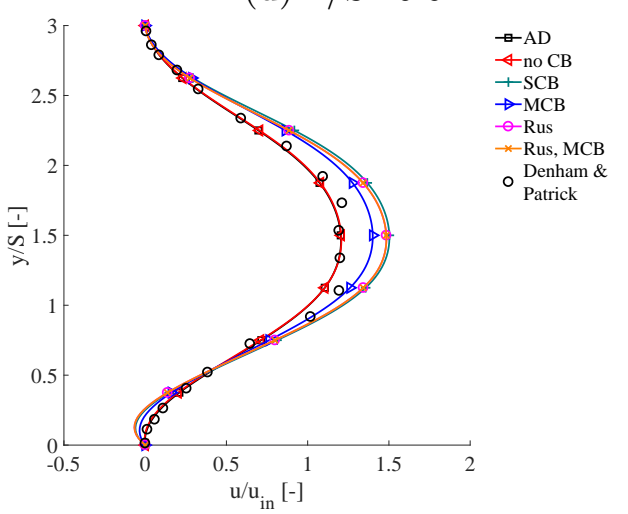

(d) $\mathrm{x} / \mathrm{S}=6.0$

(f) $\mathrm{x} / \mathrm{S}=10.0$

Figure 14: Velocity profiles for the backward facing step problem at different locations $\mathrm{x} / \mathrm{S}$ using the $\mathrm{AC}$ method at $\mathrm{Re}=229$. The reference data is taken from Denham and Patrick [67]. 

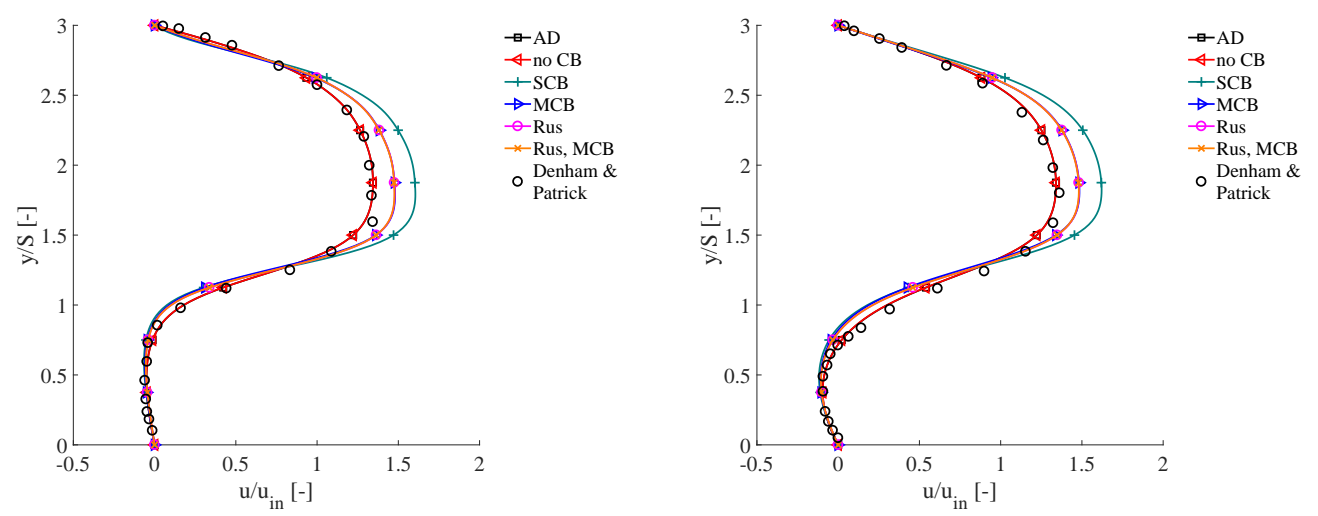

(a) $\mathrm{x} / \mathrm{S}=0.8$
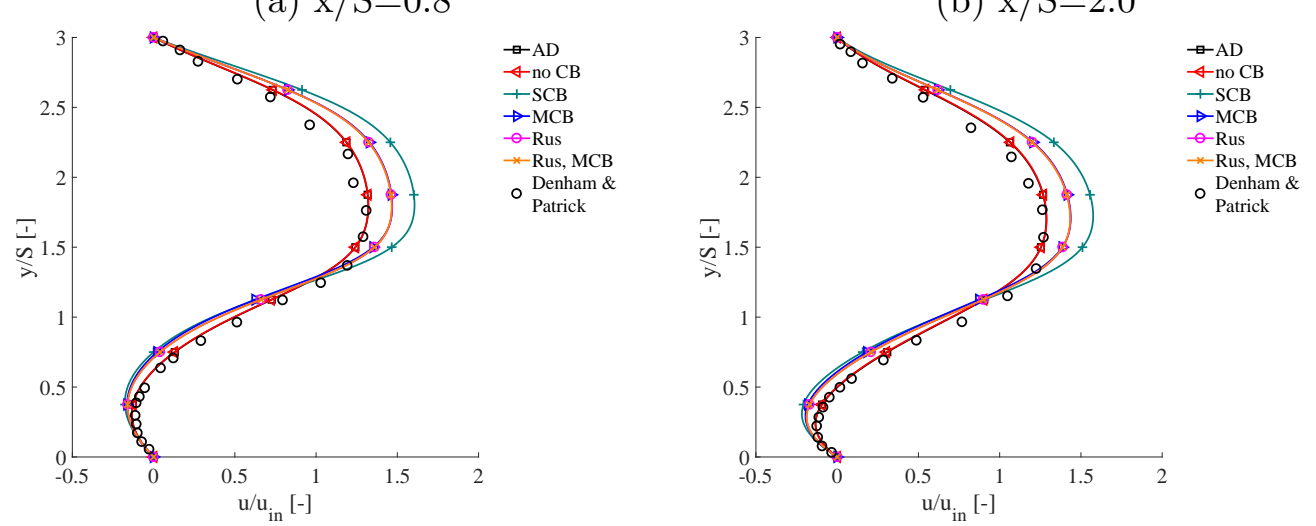

(c) $\mathrm{x} / \mathrm{S}=4.0$

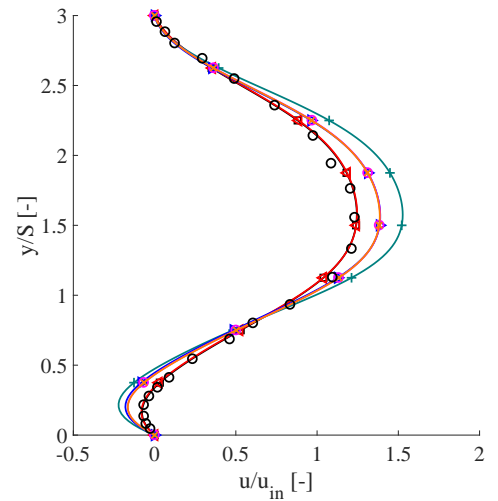

(e) $\mathrm{x} / \mathrm{S}=8.0$

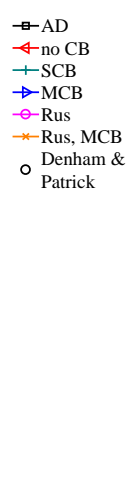

(1)

Figure 15: Velocity profiles for the backward facing step problem at different locations $\mathrm{x} / \mathrm{S}$ using the FSAC-PP method at $\mathrm{Re}=229$. The reference data is taken from Denham and Patrick [67]. 
Table 9: Comparison of the reattachment length at different Reynolds numbers. Results shown are obtained with the AC method.

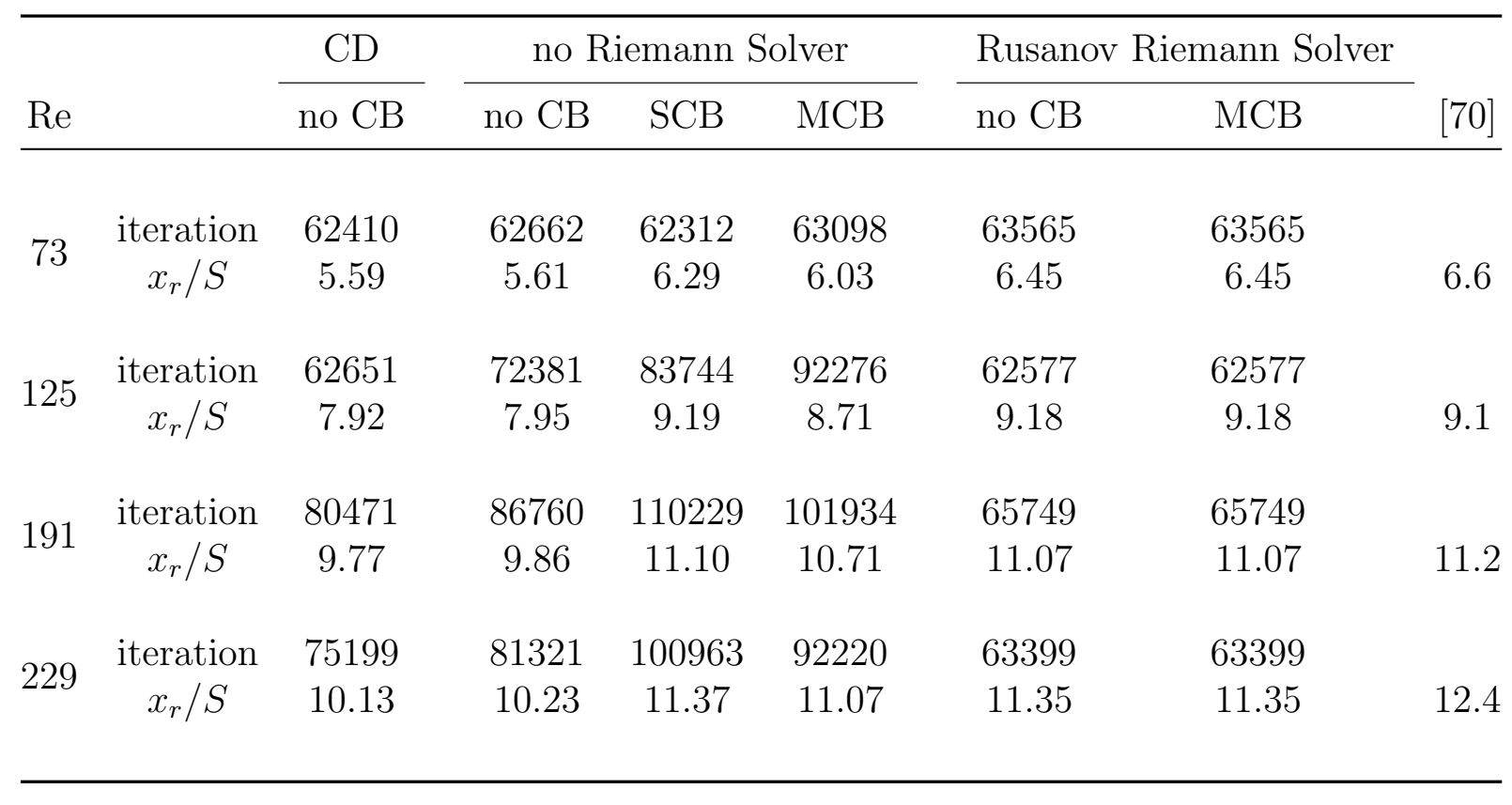

We chose to highlight the flow at $R e=229$ as Zamzamian and Razavi showed velocity profiles at the same Reynolds number. However, their implementation of the SCB scheme showed significantly different results compared to the MCB scheme, concluding that the MCB scheme shows superior performance over the SCB scheme. We cannot confirm the same findings and in-fact can see that the SCB and MCB scheme show very similar trends. The velocity profiles presented by Zamzamian and Razavi [30] showed that the velocity on the wall at the step was not set to zero, which was effectively replacing the wall with an open boundary condition in a numerical sense. It was further mentioned by Zamzamian and Razavi that the central scheme with artificial dissipation was too unstable to obtain converged results. This is in contrast with our findings, where we showed that the central scheme is matching the reference data in this case best, along with the non-CB scheme.

Table 9 and 10 show results for the different CB scheme and Riemann solver combinations for all four Reynolds numbers provided by Denham and Patrick [67]. Here, we show results of the number of iterations and reattach- 
Table 10: Comparison of the reattachment length at different Reynolds numbers. Results shown are obtained with the FSAC-PP method.

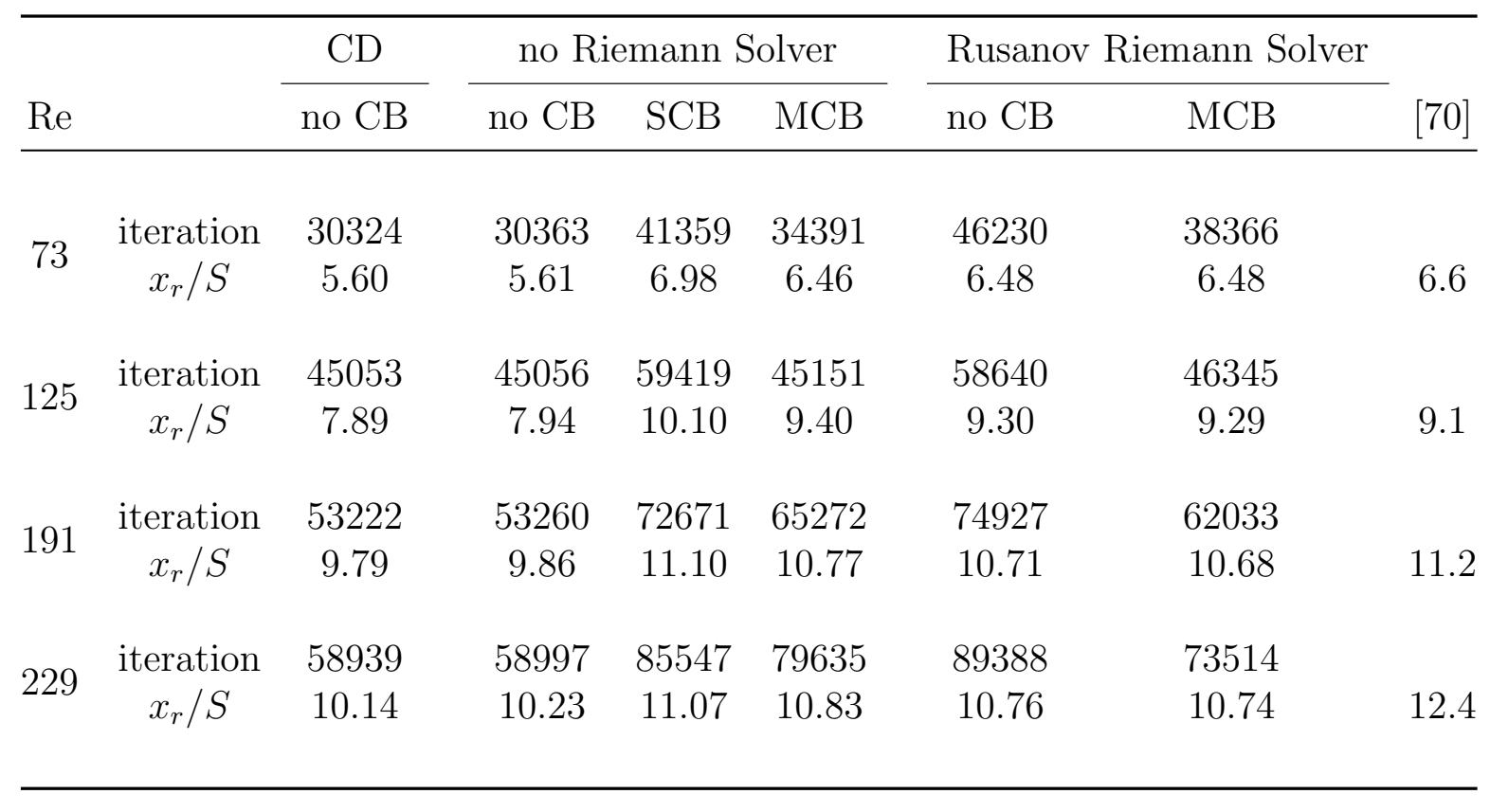

ment point as no further tabulated data for the velocity profiles are available. We can make similar observations as in the previous Sections. The number of iterations are again comparing favourably for the FSAC-PP method, where we can see a speed-up over the AC method of up to a factor of two. We need to stress here that we used the same boundary conditions as Zamzamian and Razavi who used a fixed pressure outlet boundary condition while they applied a Neumann condition for the velocity. This particular choice combined with the upstream inlet boundary condition poses its own challenges from a numerical point of view. Due to the elliptic nature of the pressure Poisson solver in the second Fractional-Step of the FSAC-PP method, the instantaneous pressure propagation results in backscatter at the outlet which induces pressure waves that travel backward through the domain. Those pressure waves cannot propagate upstream through the smaller channel as it has been removed from the simulation. Therefore, the pressure reflects at this boundary, interacting with itself and the velocity field which introduces an oscillatory behaviour and may prohibit convergence at higher Reynolds numbers. To circumvent this problem, the smaller inlet section is required so 
as to allow the pressure to develop correctly without disturbing the velocity field. Our experience shows, that a fully Neumann boundary condition at the outlet for the pressure can reduce the backscattering and thus enhancing convergence. However, the pressure is then required to be fixed by some other means in order to avoid the singularity of the solution matrix which arises for a fully Neumann boundary problem. To overcome this problem and to be consistent with the reference data against which comparisons are made, we impose a fixed pressure boundary condition at the outlet.

To assess the accuracy of the predicted reattachment length from our simulations, we compare the results against data provided by Leal and Acrivos [70], which was also used as a comparison by Denham and Patrick. The data provided by Leal and Acrivos was obtained without a top wall which replaced this physical boundary by the freestream velocity, so the results are only given here as a reference. It can be seen, however, that the Rusanov Riemann solver with and without the MCB scheme provides consistently the best agreement with the reference data, closely followed by the SCB scheme. This shows that the Riemann solver may indeed improve accuracy. Furthermore, the convergence rate has also been positively affected by the Riemann solver treatment. For the $\mathrm{AC}$ method, it can be seen that the number of residuals are lowest by using the Rusanov Riemann solver with or without the MCB scheme, apart from the $\mathrm{Re}=73$ case where the non-CB, SCB and central scheme with artificial dissipation perform marginally better. There are significant performance differences to be gained by the Rusanov approach, where at $\mathrm{Re}=191$, for example, a reduction in the number of iterations was observed to be as high as $35.5 \%$ and $40.4 \%$ compared to the MCB and SCB scheme, respectively. Although identical results were obtained in terms of number of iterations for the Rusanov Riemann solver with and without the MCB scheme, the latter provides an approximate $20 \%$ over the former when using the FSAC-PP method. Using the FSAC-PP method, this scheme may not perform always best in terms of iterations, however, it shows closer agreement with the reference data over a scheme with slightly less iterations. With increasing Reynolds number, it is also evident that the Rusanov and MCB scheme combination becomes faster than the MCB scheme by itself in terms of iterations. This again hints the performance benefits that may be derived from this scheme combination for high Reynolds number flows.

In addition to the above discussion, we also show our predicted reattachment lengths for all four Reynolds numbers using the MCB scheme without the Rusanov Riemann solver and compare these with the reattachment lengths 


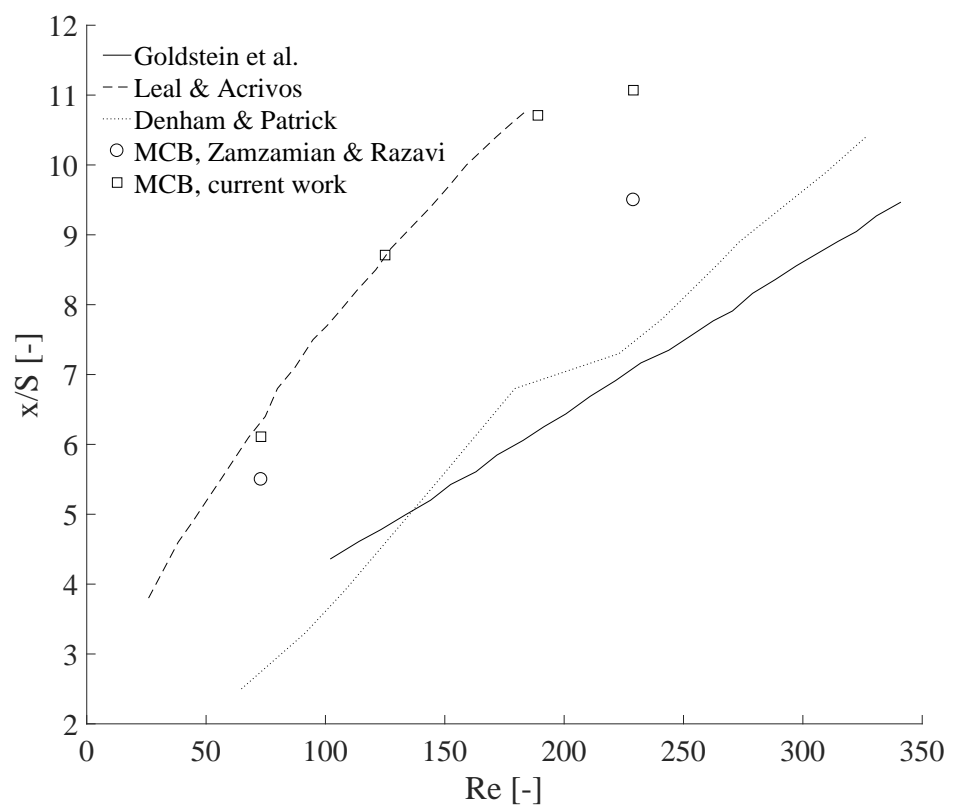

Figure 16: Comparison of the reattachment length of our implementation of the MCB scheme with that of Razavi and Zamzamian [29]. Experimental data provided by Denham and Patrick [67], Lean and Acrivos [70] and Goldstein et al. [71] are given as a reference.

given in [67]. We further compare them with other experiments cited by Denham and Patrick and show an approximate measure of the reattachment length obtained by Razavi and Zamzamian [29] for the MCB scheme, which was deducted from streamline plots which is therefore only approximate. This is shown in Figure 16.

We can see that the geometrical similar experiments of Goldstein et al. [71] and Denham and Patrick [67] provide similar data while our results compare more favourable with the work of Leal and Acrivos [70]. At lower Reynolds numbers, the reattachment length obtained by Razavi and Zamzamian compares well with our MCB scheme. At higher Reynolds numbers, however, their results are midway between our prediction and the experiments of Denham and Patrick. Keeping in mind that in their simulation fluid was allowed to exit the domain at the step, momentum was removed from the flow which thus had less energy and attached earlier to the wall than in our simulations. The fact that there is a large discrepancy between the simulations and experimental data, shows that a comparison for this geometry becomes problematic, if a non-fully developed velocity profile has 
to be imposed at the intersection of the smaller and larger channel, as was discussed earlier.

Although we hope to have convey the intrinsic difficulties attached to the reference data and numerical set up, we decided to include these results here to show that the SCB scheme does not perform as poorly as claimed by Zamzamian and Razavi compared to the MCB scheme. Furthermore, the fact that we did not observe any difficulties in getting converged results with the central scheme with artificial dissipation which was reported by Zamzamian and Razavi to "show remarkable instabilities" [30, p. 8706], combined with the fact that the velocities were not set to zero at the wall, suggests to us that there might be issues with their particular implementation. Any results about the performance of the MCB scheme against the SCB scheme are therefore misleading and in order to provide a fair comparison, reference data with a fully developed velocity profiles at the larger channel's inlet should be used for comparison purposes.

\section{Conclusion}

In the present work, a generalised multi-directional characteristics-based (MCB) scheme has been proposed which is applicable to any hyperbolic incompressible flow method. Furthermore, we have modified the Rusanov Riemann solver in a geometrical sense so that it is directly applicable to the novel MCB scheme. This approach is different from the literature on multidirectional Riemann solvers, see for example [72, 73, 74], where the Riemann solver itself is made multi-directional. In the current approach, the multidirectional nature is provided through the MCB scheme while the Riemann solver provides additional numerical dissipation that scales with the Reynolds number. In this way, a low-dissipative interpolation scheme can be used to reconstruct the inter-cell values of the primitive variables which otherwise may become unstable and non-physical. Thus, the order of the scheme can be set arbitrarily high to control the accuracy while the Riemann solver provides the stability. The MCB scheme, on the other hand, is able to resolve anisotropic behaviour better than the single-directional characteristics-based (SCB) scheme and thus may be more favourable at higher Reynolds numbers where the flow becomes turbulent. This allows the splitting of accuracy, stability and sound physical description and thus each part can be modified separately to adapt to a given flow problem. Through the addition of the Rie- 
mann solver, we have created a generalised multi-directional Godunov-type framework for incompressible flows, which, through the geometrical modification of the Riemann solver, can also be used for unstructured meshes without modifications. The scheme has been applied to the Artificial Compressibility (AC) method as a benchmark and further to the Fractional-Step, Artificial Compressibility with Pressure Projection (FSAC-PP) method which is a recent unification of Chorin's AC and Pressure Projection method.

We have tested the MCB scheme with and without a Riemann solver and compared it against the SCB and a non-CB scheme. Additional comparisons were made against the central scheme with artificial dissipation. The test cases considered were the lid driven cavity flow, the backward facing step problem and the Taylor-Green vortex flow. The latter case also allowed to make comments on the unsteady behaviour of each scheme. Overall it was found that the accuracy and potential speed-up was highly flow regime and case dependent. Unlike in the previous literature on the MCB scheme which was only used in conjunction with the $\mathrm{AC}$ method, we could not attest the same favourable convergence and accuracy properties across all methods and have shown the potential boundary condition issues which may have contributed to the biased comparisons. For laminar Reynolds numbers and steady state flows, there was little differences observed in the accuracy of the schemes. It was found, however, that the FSAC-PP method could speed-up the simulations by up to a factor of 5.8. At higher Reynolds numbers, the MCB scheme was able to delay oscillations in the pressure field more than its non-CB counterpart but required a Riemann solver to preserve stability when the Reynolds number was increased further. Interestingly, the implicit and elliptic treatment of the pressure in the FSAC-PP method showed no oscillation of the pressure and provided a mechanism to stabilise the flow. The Riemann solver was still necessary, however, to balance the lost physical dissipation through numerical dissipation. When an unsteady flow was considered, the FSAC-PP method showed further significant acceleration properties, where the average number of iterations per pseudo time step were 7-200 times lower than those obtained with the $\mathrm{AC}$ method. At the same time, the error in pressure was up to an order of magnitude lower when the same schemes at the same Reynolds number were compared between the AC and FSAC-PP method. The role of the upwinding of the Riemann solvers and the resulting numerical dissipation, as well as the non-conservative discretised form of the implemented equations have been discussed in conjunction with the decay of the kinetic energy, where all numerical schemes showed a steeper decay 
compared to the analytic solution. Those schemes that have shown an excess in numerical dissipation also produced larger errors in the predicted kinetic energy, while preserving stability at higher Reynolds numbers. However, we have not only shown advantages and disadvantages of the schemes but also highlighted that the accuracy and convergence rate is noticeably influenced by the underlying incompressible flow method.

\section{Acknowledgements}

The present research work was financially supported by the Centre for Computational Engineering Sciences at Cranfield University under the project code EEB6001R. We would also like to acknowledge the constructive comments of the reviewers of the CPC Journal.

[1] I. Grattan-Guinness, S. Engelsman, Hist. Math. 9 (1) (1982) 65-75.

[2] J. D. Hoffman, A. R. Maykut, J. Spacecr. Rockets 11 (10) (1974) 697704 .

[3] D. L. Marcum, J. D. Hoffman, AIAA J. 23 (10) (1985) 1497-1505.

[4] M. C. Cline, J. D. Hoffman, J. Comput. Phys. 12 (1) (1973) 1-23.

[5] C. Ferrari, J. Aeronaut. Sci. 16 (7) (1949) 411-434.

[6] R. A. Delaney, P. Kavanagh, J. Eng. P. 98 (3) (1976) 356-363.

[7] P. I. Chushkin, O. N. Katskova, Tech. rep., NASA (1965).

[8] J. V. Rakich, J. W. Cleary, AIAA J. 8 (3) (1970) 511-518.

[9] H. Sauerwein, J. Fluid Mech. 25 (3) (1966) 17-41.

[10] J. D. Anderson, McGraw-Hill Education, New York, 2002.

[11] M. J. Zucrow, J. D. Hoffman, John Wiley \& Sons, Inc., New York, 1977.

[12] R. A. Delaney, Ph.D. thesis, Iowa State University (1974).

[13] M. C. Cline, J. D. Hoffman, AIAA J. 10 (11) (1972) 1452-1458. 
[14] V. H. Ransom, J. D. Hoffman, H. D. Thompson, AIAA J. 10 (12) (1972) $1573-1581$.

[15] P. Roe, Annu. Rev. Fluid Mech. 18 (1) (1986) 337-365.

[16] M. J. Zucrow, J. D. Hoffman, John Wiley \& Sons, Inc., New York, 1976.

[17] H. Sauerwein, Ph.D. thesis, Massachusetts Institute of Technology (1964).

[18] V. V. Rusanov, Noi Mathematiki Mathematicheskoi Fiziki 3 (3) (1963) $508-527$.

[19] A. J. Chorin, J. Comput. Phys. 2 (1967) 12-26.

[20] D. Drikakis, P. A. Govatsos, D. E. Papantonis, Int. J. Numer. Meth. Fl. 19 (1994) 667-685.

[21] A. Eberle, Tech. rep., Von Karman Inst. (1987).

[22] P. Neofytou, J. Comput. Phys. 222 (2) (2007) 475-484.

[23] X. Su, Y. Zhao, X. Huang, J. Comput. Phys. 227 (1) (2007) 1-11.

[24] E. Shapiro, D. Drikakis, J. Comput. Phys. 210 (2) (2005) 584-607.

[25] E. Shapiro, D. Drikakis, J. Comput. Phys. 210 (2) (2005) 608-631.

[26] O. C. Zienkiewicz, R. Codina, Int. J. Numer. Meth. Fl. 20 (8-9) (1995) 869-885.

[27] O. C. Zienkiewicz, K. Morgan, B. V. K. Satya Sai, R. Codina, M. Vasquez, Int. J. Numer. Meth. Fl. 20 (8-9) (1995) 887-913.

[28] P. Nithiarasu, C.-B. Liu, Computer Meth. in Appl. Mech. and Eng. 195 (23-24) (2006) 2961-2982.

[29] S. E. Razavi, K. Zamzamian, A. Farzadi, Int. J. Numer. Meth. Fl. 57 (8) (2008) 929-949.

[30] K. Zamzamian, S. E. Razavi, J. Comput. Phys. 227 (19) (2008) 8699 8713. 
[31] R. Fathollahi, K. Zamzamian, Int. J. Numer. Meth. Fl. 76 (10) (2014) $722-736$.

[32] M. Hashemi, K. Zamzamian, Appl. Math. Comput. 230 (2014) 248-258.

[33] K. Zamzamian, M. Y. Hashemi, Appl. Math. Model. 39 (22) (2015) 7032-7044.

[34] M. Hashemi, K. Zamzamian, J. Comput. Appl. Math. 259 (2014) 752759 .

[35] S. E. Razavi, T. Adibi, J. Appl. Fluid Mech. 9 (3) (2016) 1135-1146.

[36] S. E. Razavi, M. Hanifi, J. Appl. Fluid Mech. 9 (4) (2016) 1579-1590.

[37] L. Könözsy, Ph.D. thesis, Cranfield University (2012).

[38] L. Könözsy, D. Drikakis, Commun. Comput. Phys. 16 (5) (2014) 11351180 .

[39] A. J. Chorin, Math. Comput. 22 (104) (1968) 745-762.

[40] R. Témam, Arch. Ration. Mech. An. 33 (1969) 377-385.

[41] L. Könözsy, D. Drikakis, in: European Congress on Comput. Meth. in Appl. Sci. and Eng. (ECCOMAS), Vienna, Austria, 2012.

[42] L. Könözsy, D. Drikakis, M. Ashcroft, A. Dixon, J. Persson, in: 8th European Symp. on Aerothermodynamics for Sp. Vehicles, Lisbon, Portugal, 2015.

[43] P. Tsoutsanis, I. W. Kokkinakis, L. Könözsy, D. Drikakis, R. J. Williams, D. L. Youngs, Comput. Meth. Appl. M. 293 (2015) 207-231.

[44] T.-R. Teschner, L. Könözsy, K. W. Jenkins, in: MultiSci. - XXX. microCAD Int. Multidisciplinary Scientific Conference, Miskolc, Hungary, 2016.

[45] K. Smith, T.-R. Teschner, L. Könözsy, in: MultiSci. - XXXI. microCAD Int. Multidisciplinary Scientific Conference, Miskolc, Hungary, 2017.

[46] A. Jameson, in: AIAA 10th Comput. Fl. Dyn. Conference, Honolulu, USA, 1991. 
[47] D. Drikakis, W. Rider, Springer, Heidelberg, 2005.

[48] A. Jameson, W. Schmidt, E. Turkel, in: AIAA 14th Fl. and Plasma Dyn. Conference, Palo Alto, California, USA, 1981.

[49] J. Blazek, Elsevier, Oxford, 2006.

[50] A. Pentaris, K. Nikolados, S. Tsangaris, Int. J. Numer. Meth. Fl. 19 (11) (1994) 1013-1038.

[51] G.-S. Jiang, C.-W. Shu, J. Comput. Phys. 126 (1) (1996) 202-228.

[52] K. A. Hoffmann, S. T. Chiang, Eng. Education Syst., Wichita, 1993.

[53] S. K. Godunov, Math. USSR SB+ 47 (1959) 271-306.

[54] V. V. Rusanov, USSR Comp. Math. Math+ 1 (2) (1961) 304-320.

[55] S. F. Davis, SIAM J. Sci. Stat. Comp. 9 (3) (1988) 445-473.

[56] U. Ghia, K. N. Ghia, C. T. Shin, J. Comput. Phys. 48 (3) (1982) $387-$ 411.

[57] E. Erturk, Int. J. Numer. Meth. Fl. 60 (3) (2009) 275-294.

[58] A. Leroy, M. D. Violeau, C. Ferrand, C. Kassiotis, J. Comput. Phys. 261 (2014) 106-129.

[59] M. El Rafei, L. Könözsy, Z. Rana, Aerosp. 4 (4) (2017) 59.

[60] O. San, K. Kara, Computers and Fl. 117 (2015) 24-41.

[61] D. Drikakis, C. Fureby, F. F. Grinstein, D. Youngs, J. Turbul. 8 (July 2013) (2007) N20.

[62] M. E. Brachet, M. Meneguzzi, H. Politano, P. L. Sulem, J. Fl. Mech. 194 (1988) 333-349.

[63] G. Oger, S. Marrone, D. L. Touz, M. de Leffe, J. Comput. Phys. 313 (2016) 76-98.

[64] A. Khayyer, H. Gotoh, Y. Shimizu, J. Comput. Phys. 332 (2017) 236256. 
[65] J. A. Ekaterinaris, Prog. Aerosp. Sci. 41 (3) (2005) 192-300.

[66] Y. Morinishi, T. Lund, O. Vasilyev, P. Moin, J. Comput. Phys. 143 (1) (1998) 90-124.

[67] M. Denham, M. Patrick, Chem. Eng. Res. Des. 52 (1974) 361-367.

[68] F. Durst, S. Ray, B. Unsal, O. A. Bayoumi, J. Fluids Eng. 127 (6) (2005) 1154.

[69] P. J. Roache, J. Fluids Eng. 116 (3) (1994) 405.

[70] L. G. Leal, A. Acrivos, J. Fluid Mech. 39 (04) (1969) 735-752.

[71] R. J. Goldstein, V. L. Eriksen, R. M. Olson, E. R. G. Eckert, J. Basic Eng. 94 (4) (1970) 732-739.

[72] D. S. Balsara, J. Comput. Phys. 229 (6) (2010) 1970-1993.

[73] D. S. Balsara, J. Comput. Phys. 231 (22) (2012) 7476-7503.

[74] D. S. Balsara, M. Dumbser, R. Abgrall, J. Comput. Phys. 261 (2014) 172-208. 


\title{
A generalised and low-dissipative
} multi-directional characteristics-based scheme with inclusion of the local Riemann problem investigating incompressible flows without free-surfaces,

\author{
Teschner, Tom-Robin
}

Elsevier

Teschner T-R, Könözsy L, Jenkins KW. A generalised and low-dissipative multi-directional characteristics-based scheme with inclusion of the local Riemann problem investigating incompressible flows without free-surfaces. Computer Physics Communications, Volume 239, June 2019, pp. 283-310

https://doi.org/10.1016/j.cpc.2018.07.026

Downloaded from Cranfield Library Services E-Repository 